\title{
Thermal and Hydrodynamic Characteristics Research of a Tree-Shaped Microchannel Network Thermal Exchanger on the High-Speed Motorized Spindle
}

Song-Hua Li ( $\square$ lisonghua@sjzu.edu.cn )

Shenyang Jianzhu University https://orcid.org/0000-0002-6487-6779

Zi-Chen Zhao

Shenyang Jianzhu University

Yong-Hua Wang

Shenyang Jianzhu University

Wei-Nan Wang

Shenyang Jianzhu University

\section{Original Article}

Keywords: Thermal energy optimization, Hydrodynamic characteristics, Tree-shaped microchannel network, Spindle thermal exchanger, Coefficient of performance (COP)

Posted Date: December 1st, 2020

DOI: https://doi.org/10.21203/rs.3.rs-116059/v1

License: (c) (1) This work is licensed under a Creative Commons Attribution 4.0 International License.

Read Full License 


\section{Title page}

\section{Thermal and Hydrodynamic Characteristics research of a tree-shaped microchannel network thermal exchanger on the high-speed motorized spindle}

Song-Hua Li, born in 1977, is currently a professor and a PhD candidate supervisor at National-Local Joint Engineering Laboratory of NC Machining Equipment and Technology of High-Grade Stone, Shenyang Jianzhu University, China. His research interests include high performance $\mathrm{CNC}$ machine tool spindle system, high efficiency and precision machining technology and equipment for intractable materials, high-grade stone $\mathrm{CNC}$ machining equipment and technology and modern construction engineering equipment and technology. Tel: +86-024-24690319; E-mail: lisonghua@sjzu.edu.cn

Zi-Chen Zhao, born in 1995, is currently a master candidate at School of Mechanical Engineering, Shenyang Jianzhu University, China. E-mail: syjzdxzzc@ hotmail.com

Yong-Hua Wang, born in 1990, is currently a $\mathrm{PhD}$ candidate at School of Mechanical Engineering, Shenyang Jianzhu University, China.

E-mail: yonghua0514@163.com

Wei-Nan Wang, born in 1989, is currently a PhD candidate at School of Mechanical Engineering, Shenyang Jianzhu University, China. E-mail: robinwang@sjzu.edu.cn

Corresponding author: Song-Hua Li E-mail: lisonghua@sjzu.edu.cn 


\title{
Thermal and Hydrodynamic Characteristics research of a tree-shaped microchannel network thermal exchanger on the high-speed motorized spindle
}

\author{
Song-Hua $\mathrm{Li}^{1,2} \cdot \mathrm{Zi}^{-C h e n} \mathrm{Zhao}^{1} \cdot$ Yong-Hua Wang ${ }^{1} \cdot$ Wei-Nan Wang ${ }^{1}$
}

Received June xx, 201x; revised February xx, 201x; accepted March xx, 201x

(C) Chinese Mechanical Engineering Society and Springer-Verlag Berlin Heidelberg 2017

\begin{abstract}
To improve the thermal dissipation of high-speed motorized spindle, core component of machine tool, according to the construction theory of mammalian circulation and respiratory system, this paper optimizes the distribution of the existing tree-shaped microchannel network structure, changes the thermal exchanger assembly mode, directly as the spindle shell, and make it possible in engineering applications. Considered the conjugate heat transfer between the spindle stator, thermal exchanger, and cooling water, a three-dimensional (3D) fluid thermodynamic model of a tree-shaped microchannel network thermal exchanger and a conventional spiral thermal exchanger are established. Both have the identical heat exchange area and inlet size. The hydrodynamic characteristics, pressure loss, temperature gradient distribution, and coefficient of performance (COP) are compared. The results indicate that the new structure proposed in this paper has more excellent hydrodynamic characteristics, smaller pressure changes, more uniform temperature gradient distribution, and greater COP. The experiment results verify the correctness of the theoretical calculation. It has a wide range of application prospects in thermal problems of high-speed spindle.
\end{abstract}

Keywords: Thermal energy optimization - Hydrodynamic characteristics - Tree-shaped microchannel network - Spindle thermal exchanger $\bullet$ Coefficient of performance (COP)

\section{Introduction}

In today's competitive marketplace, machine tool industry

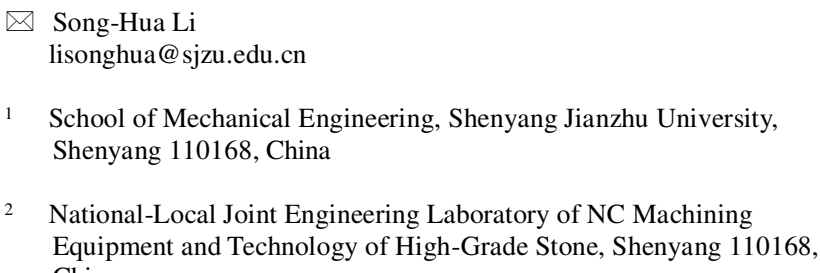

2 National-Local Joint Engineering Laboratory of NC Machining Equipment and Technology of High-Grade Stone, Shenyang 110168, China of high-end numerical control is at a critical stage in high-precision manufacturing, green manufacturing and intelligent manufacturing. The thermal dissipation of machine tools has become an urgent problem. As the machine tool's core component, the high-speed motorized spindle has become a severe area of machine tool thermal problems due to its compact structure and high speed. The positioning uncertainty of the machine tool caused by thermal issues directly affects the parts' dimensional accuracy [1]. Up to $75 \%$ of the geometric error of the processed workpiece is caused by temperature [2]. The thermal energy of high-speed spindle system is mainly from the motor and the bearing. However, the critical factor affecting working accuracy of high-speed spindle system is not the temperature rise but the thermal field distribution. In other words, it is the periodicity and the uniformity of the thermal field distribution. Therefore, how to improve the heat exchange efficiency and optimize its thermal field distribution has become the central issue explored in this article. As the "volumetric specific power" of the high-speed motorized spindle is relatively high and the "high-temperature area" of high-speed spindle is mainly concentrated in the front bearing and motor part, active cooling is usually required. The most effective cooling method is to set a continuous liquid coolant around the heat source [3-4]. Donmez[5] proposed a low-cost, abnormal shape silicon tube with small crevices and using compressed air to stabilize the temperature to reduce the machine tool's thermal error, which improved the thermal displacement by $30 \%$. Nevertheless, the cooling effect of air is not as good as water.

Many ways to optimize engineering problems were found in nature. The blood circulation system and respiratory system of mammals have advanced heat and 
material transfer efficiency. This geometric structure is the result of evolution in nature, and its structural theory is the primary goal of research. The geometric structure constructed according to the structural theory may obtain advanced heat and material transfer performance. This structure has essential applications in the fields of electronic equipment cooling and fuel cell material transfer. The tree-shaped microchannel network thermal exchanger was researched and developed to improve the cooling system's material transfer efficiency and the spindle system's thermal characteristics. Bejan [6] proposed a method of collecting and "directing" the generated heat to a point under a low conductivity volume of a given size. To minimize flow resistance, Bejan [7] theoretically determines each geometric detail and optimizes the component shape and pathwidth. These paths form completely deterministic tree networks. Subsequently, Bejan [8-9] gave the principles of natural and engineering construction.

Rectangular fractal tree-shaped microchannel network has been researched and developed, but the existing structural theory is not applicable for disc-shaped electronic chip. Wechsatol et al. [10] designed a disc-shaped fractal tree-shaped microchannel network with minimal flow resistance. To minimize the fluid flow pumping power in a disk-shaped tree network, Gosselin [11] optimized a tree-shaped structure, which can provide a design basis for the tree-shaped network.

Based on the fractal structure theory proposed by Bejan, Chen and Cheng et al. [12] studied tree-like microchannel networks to rectangular electronic chip cooling. Using advanced MEMS technology and fractal theory, electronic chips on silicon wafers were also cooled [13]. The experimental results showed that the fractal tree-like microchannel network has incomparable heat transfer efficiency compared with the conventional parallel microchannel under the same experimental conditions. Senn [14] applied the tree-like microchannel nets to improve the fluid distribution of fuel cells. The geometry of the polymer electrolyte fuel cell was optimized. It is found that the performance of the battery can be significantly improved by using the tree-like nets instead of the conventional snake channel in the rectangular system. Senn [15] further optimized the fractal design and applied the optimization results to the DMFC to obtain higher electrical density and net power density.

Alharbi and Pence [16] used 3D computational fluid dynamics to study the flow in fractal networks. The maximum temperature and pressure drop were compared between the fractal tree-shaped microchannel and the disk-shaped array straight channel. The results showed that the fractal tree-shaped microchannel network can provide a better cooling effect [17].

According to the restudied Murray's law of laminar flow [18], Jing et al. [19] optimized and analyzed the fractal tree-like microchannel network's electro-viscous flow consideration the surface charge and reached the minimum hydraulic resistance under the constraint of constant channel volume. Then they theoretically and numerically studied the electro-osmotic flow of the tree-like branching microchannel network [20].

Xia [21] designed a new type of fractal tree-like channels network heat sink for high-speed motorized spindle based on the fractal theory. Considering the microchannel wall's conjugate heat transfer, the heat transfer and pressure drop were numerically studied and verified by experiments. The results showed that the designed fractal tree-like microchannel network thermal exchanger has less pressure loss, more evenly distribution of the thermal field and greater COP than the conventional spiral channel network thermal exchanger.

The tree-shaped microchannel networks do not cover the thermal exchanger completely. The temperature gradient distribution is nonuniform. Whether the hydrodynamic characteristics of the arc-shaped tree-shaped network are affected. The three layers composite structure of fractal tree-shaped microchannel network thermal exchanger requires high machining precision and assembly of each layer, which increase the difficulty of assembly and the machining cost of motorized spindle. There is virtually no possibility of practical application. These issues still need to be explored and verified, and it is still far from practical application.

Therefore, this paper optimized a tree-shaped microchannel network's distribution on thermal exchanger of high-speed motorized spindle and structured a 3D model. By changing the thermal exchanger structure, the assembly method was optimized, therefore the machining precision requirement was reduced and the cost saving goal was achieved. The numerical analysis considered the conjugate heat transfer of the thermal exchanger. The hydrodynamic characteristics at the branch of the arc-shaped tree-shaped microchannel network are numerical analyzed and discussed. The temperature gradient of the thermal exchanger is identified and compared. The theoretical analysis result is checked by the experiment.

\section{Thermal exchanger design}

For the rectangular tree-shaped microchannel network 
thermal exchanger, two main structures have been built [22], as shown in Figure 1. The main difference between these two tree-shaped structures is the angle between the branches. In Figure 1(a), the branch angle is $90^{\circ}$, and in Figure 1(b), the branch angle is $180^{\circ}$.

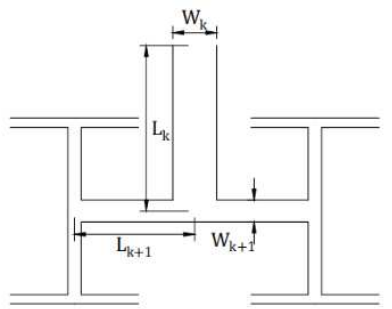

(a)

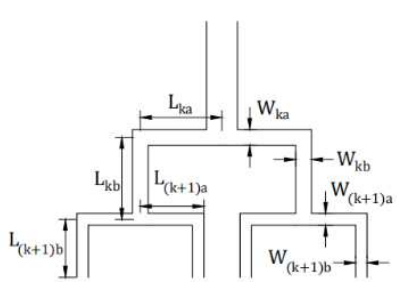

(b)
Figure 1 Two tree-shaped structures (a) The branch angle of $90^{\circ}$ (b) The branch angle of $180^{\circ}$

Based on the design concept of C. Xia [21], considering the particularity of the application object and the limitations of the structure in Figure 1(b), a tree-shaped structure with a branch angle of $90^{\circ}$ is adopted. As shown in Figure 1(a).

To generate the rectangular tree-shaped microchannel network in Figure 1(a), its geometric size should meet the following law of proportion [6]:

$$
\begin{aligned}
& \frac{L_{k+1}}{L_{k}}=\gamma, \\
& \frac{D_{k+1}}{D_{k}}=\beta,
\end{aligned}
$$

In this formula, $L_{k}$ represent the $k$-th channel length and $D_{k}$ represent the $k$-th channel hydraulic diameter, $k$ is the natural number. $\gamma$ and $\beta$ are constants, determined by the equations given by B.B. Merbrot [23]:

$$
\begin{aligned}
& \gamma=N^{-1 / D}, \\
& \beta=N^{-1 / 4},
\end{aligned}
$$

Where $N$ is expressed as the number of the next-level branch channels appearing at the end of the channel. As shown in Figure 1(a), the next-level channel is split into two branches, so $N=2$. $D$ is the channel length distribution size. According to the research of Chen YP [12], when $D=2$, the heat transfer efficiency is the highest, so $D=2$ is selected. $\Delta$ is the channel diameter. Murray [24] studied the optimal vascular diameter of blood flow in the vascular system, which is 3 . The diameter of the human lung tube trees which indicated by Mandelbrot [23] is 3. Bejan [25] proposes a $2^{1 / 3}$ tree diameter ratio that is best suited to laminar flow. Therefore $\Delta=3$.

In Figure 1(a), the lateral section of the channel is rectangle, and the $W_{k}$ is the $k$-th branch width. Therefore, the hydraulic diameter $D_{k}$ is expressed as:

$$
D_{k}=\frac{4 W_{k} h}{2\left(W_{k}+h\right)}
$$

Where $h$ is the channel depth.

The thermal exchanger of high-speed spindle is cylindrical. If the tree-shaped microchannel network in Figure 1(a) is to be evenly distributed on the cylindrical thermal exchanger, the tree-shaped microchannel must be adjusted in structure. The adjusted tree-shaped microchannel network structure is shown in Figure 2. This figure shows channels can be divided into axial channels and circumferential channels according to the distribution direction.

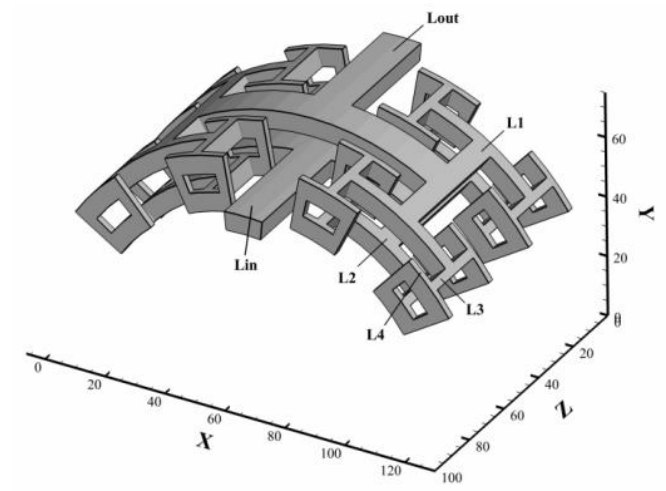

Figure 2 Cylindrical tree-shaped microchannel network structure

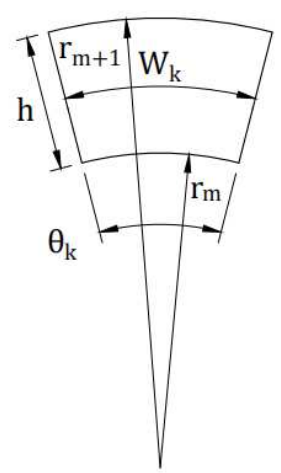

(a)

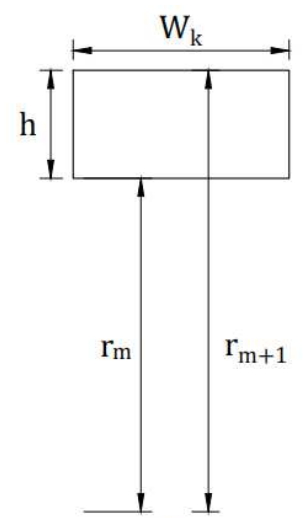

(b)
Figure 3 Lateral section shape (a) Axial channel lateral section (b) Circumferential channel lateral section

The axial channel length is denoted by $L_{k}$ ( $k$ is odd) and $L_{k}$ is linear. The lateral section shape is shown in 
Figure 3(a). The central angle of the lateral section is represented by $k$ ( $k$ is odd).

The circumferential channels length is denoted by $\dot{L}_{k}$ (k is even) and $\dot{L}_{k}$ is arc. The lateral section shape is shown in Figure 3(b). $r_{m+1}$ is the outside diameter; $r_{m}$ is the inside diameter $(m=1,3)$.

The channel length of the tree-shaped microchannel network thermal exchanger shall meet the following conditions:

$$
\begin{gathered}
L_{i n}=\frac{L}{2}, \\
W_{i n}=W_{0}, \\
L_{1}+L_{3}+L_{2 m-1}+\mathrm{L}+L_{k}<\frac{L}{2}(k=2 m-1), \\
L_{0}+L_{2}+\dot{L}_{2 m}+\mathrm{L}+\dot{L}_{k}<\frac{1}{4} \pi\left(r_{m}+r_{m+1}\right)(k=2 m), \\
L_{3}+L_{2 \mathrm{~m}-1}+\mathrm{L}+L_{k}<L_{1}(k=2 m-1), \\
\dot{L}_{2}+\dot{L}_{2 m}+\mathrm{L}+\dot{L}_{k}<\dot{L}_{0}(k=2 m),
\end{gathered}
$$

Equation (6) determines the length of the afflux channel. Equation (7) determines the channel width. Inequalities (8) and (9) make the channel sufficiently distributed on the thermal exchanger. Inequalities (10) and (11) prevent the channels from overlapping at the highest branch.

The cylindrical tubular tree-shaped microchannel thermal exchanger constructed by the above parameters is shown in Figure 4.

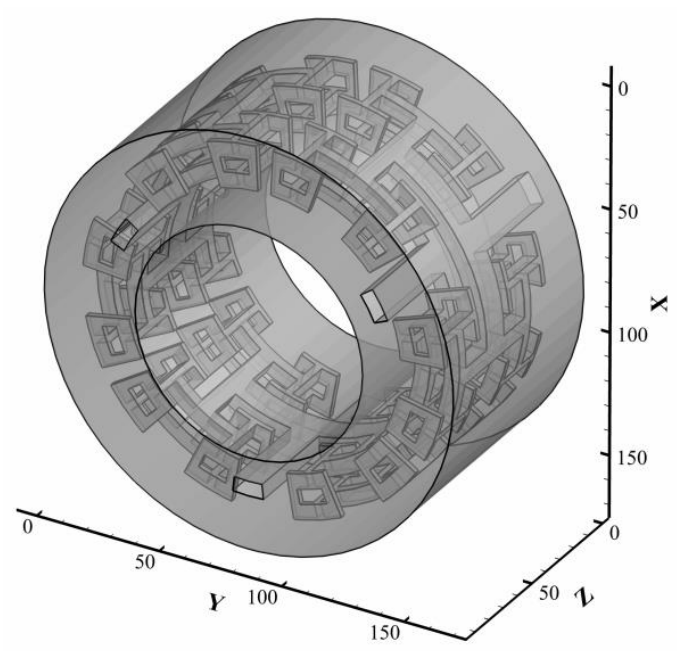

Figure 4 Cylindrical tree-shaped microchannel network thermal exchanger

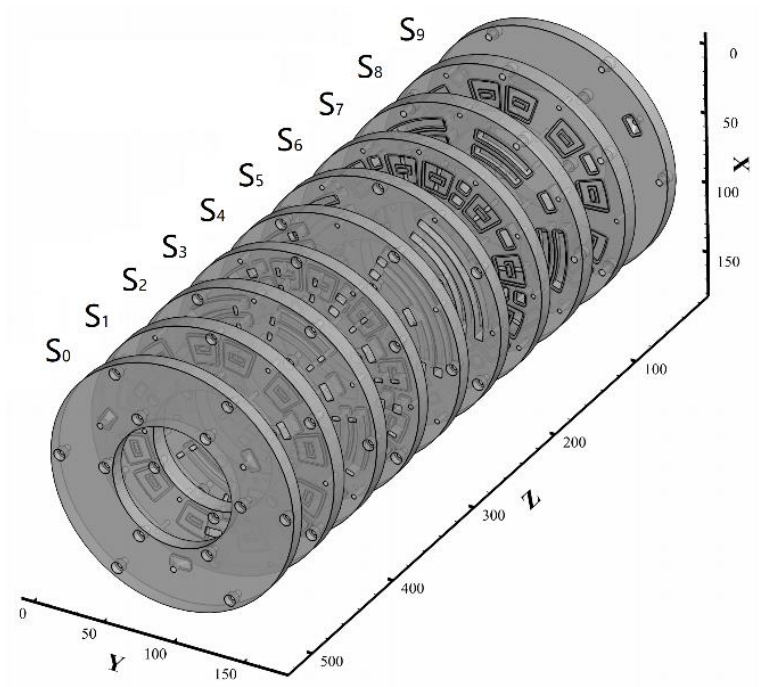

Figure 5 The segmented structure of tree-shaped microchannel network thermal exchanger

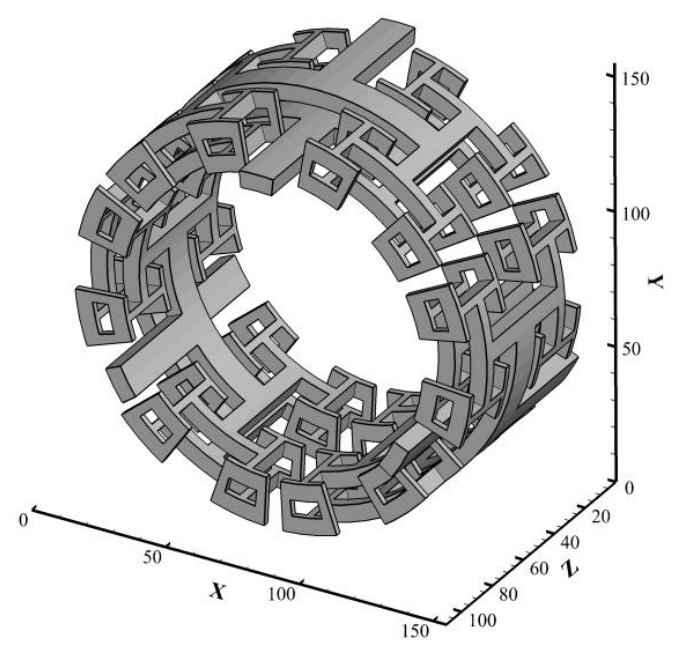

Figure 6 Tree-shaped microchannel network distribution map

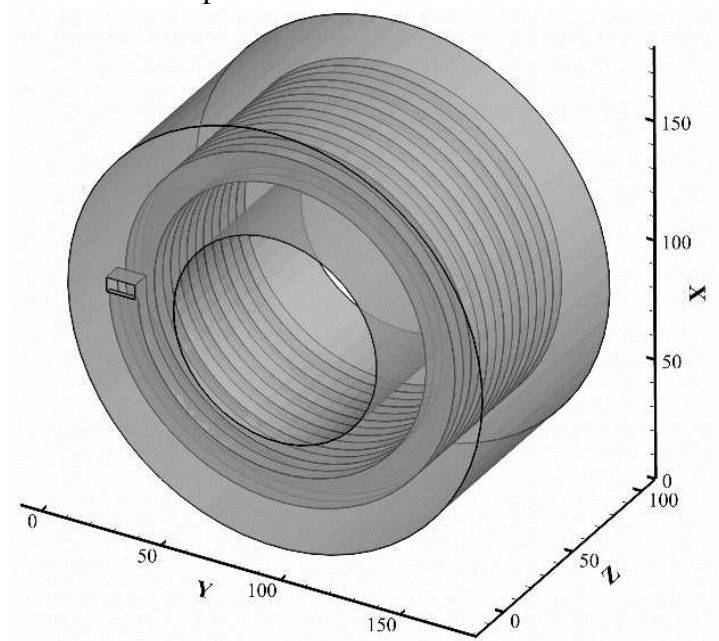

Figure 7 The conventional spiral thermal exchanger 
According to Figure 5, the cylindrical-shaped thermal exchanger's segmented structure is divided into eight segments. The segments were bolted together, and sealing rings were used on each contact surface. The length of each segment is symmetric at $Z=46 \mathrm{~mm}$. The afflux channel and the efflux channel have the same structure. The afflux channel and the efflux channel point in opposite directions. The highest branch end of the efflux channel and the afflux channel are connected by radial channels to form a complete circulation channel network. In Figure 6, a complete cylindrical thermal exchanger was constituted by three identical circulation channel networks.

Table 1 Tree-shaped microchannel network thermal exchanger structure parameters list

\begin{tabular}{|c|c|c|c|c|c|c|c|}
\hline \multicolumn{2}{|c|}{ Heat exchange area } & \multirow{2}{*}{\multicolumn{2}{|c|}{$\begin{array}{c}101963.79 \mathrm{~mm}^{2} \\
R_{1}=46.5 \mathrm{~mm} \\
R_{2}=83.5 \mathrm{~mm} \\
L=92 \mathrm{~mm}\end{array}$}} & \multirow{2}{*}{\multicolumn{2}{|c|}{ 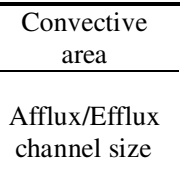 }} & \multirow{2}{*}{\multicolumn{2}{|c|}{$\begin{array}{c}133466.37 \mathrm{~mm}^{3} \\
h=6 \mathrm{~mm} \\
W=12 \mathrm{~mm}\end{array}$}} \\
\hline \multicolumn{2}{|c|}{ Thermal exchanger size } & & & & & & \\
\hline $\begin{array}{r}\text { Thern } \\
\text { segment }\end{array}$ & $\begin{array}{l}\text { exchanger } \\
\text { structure size }\end{array}$ & \multicolumn{3}{|c|}{$\begin{array}{l}S_{1}=S_{8}=12.56 \mathrm{~mm} \\
S_{2}=S_{7}=12.58 \mathrm{~mm} \\
S_{3}=S_{6}=10.42 \mathrm{~mm} \\
S_{4}=S_{5}=10.44 \mathrm{~mm} \\
S_{0}=S_{9}=10.00 \mathrm{~mm}\end{array}$} & \multicolumn{3}{|c|}{$\begin{array}{c}\text { Afflux channel size: } \\
\qquad \begin{array}{c}r_{1}=56 \mathrm{~mm} \\
r_{2}=62 \mathrm{~mm}\end{array} \\
\text { Efflux channel size: } \\
r_{3}=68 \mathrm{~mm} \\
r_{4}=74 \mathrm{~mm}\end{array}$} \\
\hline \multirow[t]{8}{*}{$\begin{array}{c}\text { channel } \\
\text { size }\end{array}$} & $k$ & in & 0 & 1 & 2 & 3 & 4 \\
\hline & $L_{k} / \mathrm{mm}$ & 46 & 32.52 & 23 & 16.26 & 11.5 & 8.13 \\
\hline & $D_{k} / m m$ & 8 & 8 & 6.32 & 4.99 & 3.94 & 3.12 \\
\hline & $\mathrm{h} / \mathrm{mm}$ & 6 & 6 & 6 & 6 & 6 & 6 \\
\hline & $W_{k} / m m$ & 12 & 12 & 6.68 & 4.28 & 2.93 & 2.18 \\
\hline & $\theta_{1} /^{\circ}$ in & 11.65 & - & 6.49 & - & 2.85 & - \\
\hline & $\sigma_{k} \quad$ out & 9.68 & - & 5.39 & - & 2.36 & - \\
\hline & $\omega_{k} / \circ$ & - & 33.58 & - & 15.79 & - & 7.90 \\
\hline
\end{tabular}

According to above theory, a tree-shaped microchannel network thermal exchanger was constructed. $L$ is the length, $2 R_{l}$ is the inside diameter, and $2 R_{2}$ is the outside diameter. Detailed structure parameters are displayed in Table 1.

In Figure 7, the conventional spiral thermal exchanger with the same channel lateral section size and heat transfer area was used as the control group. Detailed structure parameters are displayed in Table 2.

Table 2 Conventional spiral thermal exchanger structure parameters list

\begin{tabular}{cccc}
\hline Inner diameter & Outer diameter & Length & Heat exchange area \\
\hline $93 \mathrm{~mm}$ & $167 \mathrm{~mm}$ & $92 \mathrm{~mm}$ & $101983.75 \mathrm{~mm}^{2}$ \\
\hline
\end{tabular}

\section{Mathematical model}

The temperature gradient distribution and hydrodynamic characteristics of the thermal exchanger were analyzed by ANSYS-FLUENT. For the solid part, the thermal exchanger material and the heat source material are Al-alloy. Set a uniform ring-shaped heat source on the $R_{l}$ surface, the remaining faces are heat-insulated. Water liquid was selected as the fluid part. Set a constant mass flow rate at the fluid inlet, change the fluid flow velocity at the inlet and set the barometric pressure as the outlet pressure boundary condition. The fluid properties are shown in Table 3.

Table 3 Thermophysical properties of fluid

\begin{tabular}{cccc}
\hline Fluid & $\rho\left(\mathrm{kg} \cdot \mathrm{m}^{-3}\right)$ & $c_{p}\left(J \cdot \mathrm{kg}^{-1} \cdot \mathrm{K}^{-1}\right)$ & $\lambda\left(\mathrm{W} \cdot \mathrm{m}^{-1} \cdot \mathrm{K}^{-1}\right)$ \\
\hline water-liquid & 998.2 & $4.183 \times 10^{3}$ & $59.89 \times 10^{-2}$ \\
\hline
\end{tabular}

To simplify the thermodynamic model, the ideal boundary conditions were adopted:

- Stable laminar flow and heat transfer;

- The properties of solids and fluids are constant;

- Ignore gravity;

- No heat radiation and convection heat transfer.

The numerical calculation conclusions are obtained through the following governing equation:

On the fluid side

Continuity:

$$
\nabla \cdot \stackrel{1}{V}=0
$$

Law of conservation of momentum equation:

$$
\stackrel{1}{\rho_{f}}(V \cdot \nabla V)=-\nabla P+\mu_{f} \nabla^{2} V,
$$

Law of conservation of energy equation:

$$
\rho_{f} c_{p, f}(V \cdot \nabla T)=\lambda_{f} \nabla^{2} T,
$$

On the solid side

Energy equation:

$$
\lambda_{s} \nabla^{2} T=0,
$$

Boundary conditions

No sliding on the wall:

$$
u_{\Gamma}=v_{\Gamma}=w_{\Gamma}=0,
$$

Apply a uniform flow rate at the entrance of the channel: Constant heat flow on $R_{I}$ surface:

$$
u=\frac{\mu R e}{\rho_{f} D_{\text {in }}}, v=0, w=0,
$$

Inlet fluid temperature: $T_{i n}=293.15 \mathrm{~K}$

Considering the continuous coupled heat transfer between solid and fluid, solid and solid in the thermal exchanger, it can be expressed as:

$$
T_{s, \Gamma}=T_{f, \Gamma},-\lambda_{s}\left(\left.\frac{\partial T_{s}}{\partial n}\right|_{\Gamma}\right)=-\lambda_{f}\left(\left.\frac{\partial T_{f}}{\partial n}\right|_{\Gamma}\right),
$$

Different grid types and sizes were used to test grid 
independence. Experimental results show that the final calculation results are not depended on mesh type and size. The numerical analysis converges to $1 \times 10^{-6}$.

\section{Manufacture and Experimental setup}

According to the tree-shaped microchannel network thermal exchanger structure designed in this paper, two kinds of thermal exchanger structures are processed on the same Al-alloy bar using the DMG DMU50 five-axis machining center. The tree-shaped microchannel network thermal exchanger structure is shown in Figure. 8 and the setup scheme of the experimental device is shown in Figure. 9. As shown in Figure 9, the cooling water was set at $20^{\circ} \mathrm{C}$ and flowed out of the precision water cooling machine through the thermal exchanger and returned to the precision water cooling machine to complete a cooling cycle. The flow rate of cooling water was adjusted at Reynolds number 800 and 2300 by the internal valve of the precision water cooling machine. A constant heat source was provided by a uniform annular heat source inside the thermal exchanger. The thermal exchanger was coated with insulating cotton to reduce heat exchange with surrounding environment. Sensors for measuring temperature and pressure were installed at the inlet and outlet of the thermal exchanger. For the tree-shaped microchannel network thermal exchanger, $T_{1} \sim T_{3}$ temperature sensors were placed in the shell between two tree-shaped micro-channels, and $T_{4} \sim T_{6}$ temperature sensors were placed in the shell above the inlet and outlet of the tree-shaped microchannel. The specific layout is shown in Figure 10. The position of temperature sensors in the spiral thermal exchanger was the same. The temperature sensors used in this paper were M6-K, and the pressure transducers were MIK-PX300. The measured data were transmitted to a computer for processing through the Data Acquisition System.

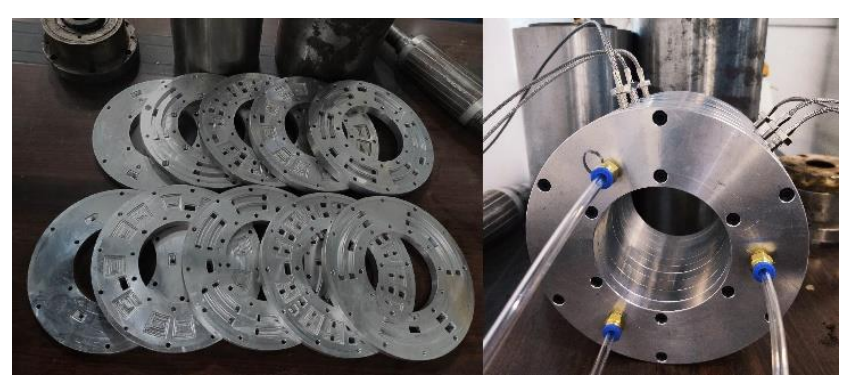

Figure 8 The tree-shaped thermal exchanger structure

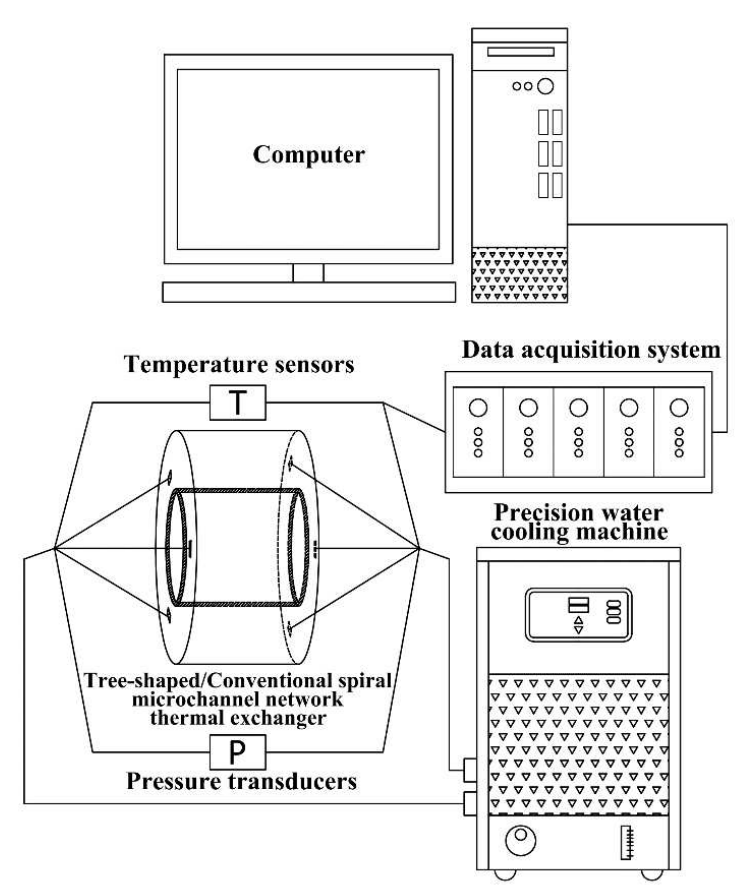

Figure 9 The setup scheme of the experimental device

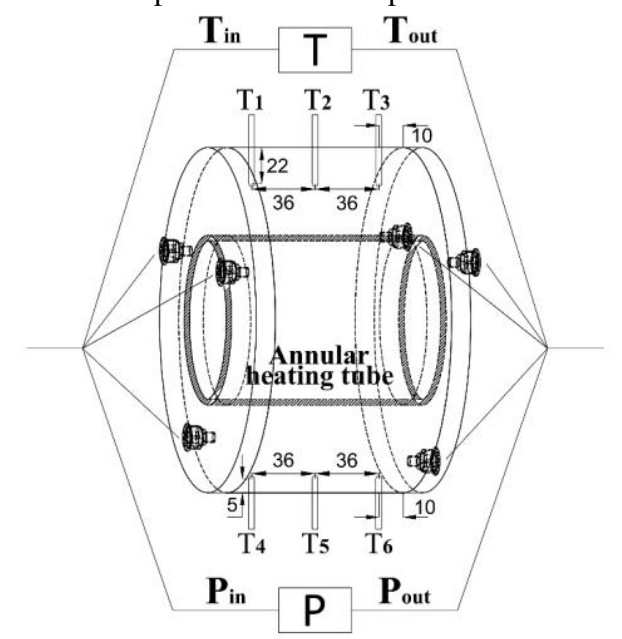

Figure 10 The specific layout of the sensors

\section{Results and Discussion}

The experimental results and the numerical analysis results were compared as shown in Table 4 and Table 5. Combined with the comparison results, the properties of the tree-shaped microchannel thermal exchanger were discussed as follows.

\subsection{Temperature gradient distribution}

The thermal exchanger temperature gradient and the uniformity of thermal field distribution directly affect the 
machining accuracy of high-speed motorized spindle. Considering the conjugate heat transfer of the wall, the thermal field of the thermal exchanger can be accurately judged. The temperature gradient is expressed by equation (12):

$$
\Delta T=T_{\max }-T_{\min },
$$

Where temperature gradient is the $\Delta T$, the thermal field maximum temperature is $T_{\max }$, the thermal field maximum temperature is $T_{\min }$.
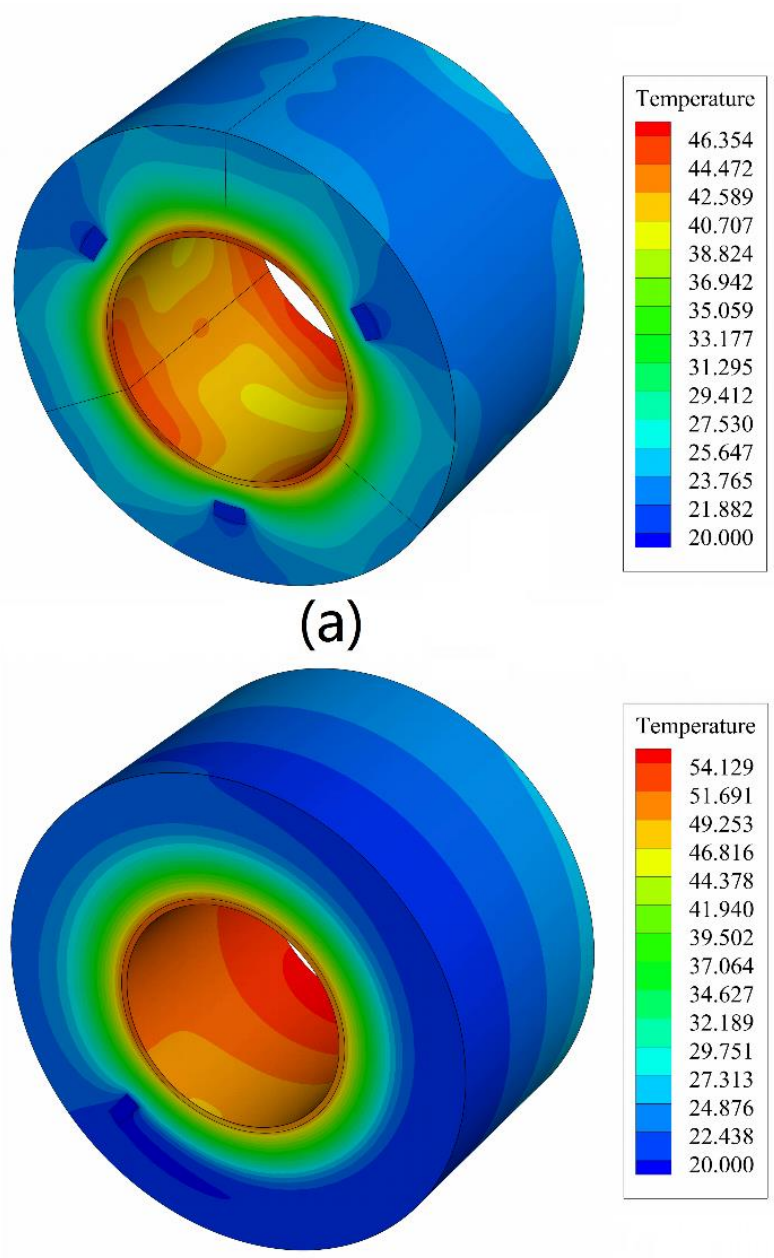

(b)

Figure 11 Comparison of thermal field distribution (a) Thermal field distribution of tree-shaped thermal exchanger (b) Thermal field distribution of conventional spiral thermal exchanger

The thermal field distribution of the tree-shaped microchannel network thermal exchanger and the conventional spiral thermal exchanger is shown in Figure 11(a) and Figure 11(b). The thermal field of the tree-shaped microchannel network thermal exchanger is more evenly distributed in the axial direction with periodicity and smaller temperature gradients (Figure 12). However, the high-temperature area of conventional spiral thermal exchanger is mainly concentrated in the thermal exchanger flow channel outlet. The temperature distribution is uneven and a massive gradient change in the axial direction.

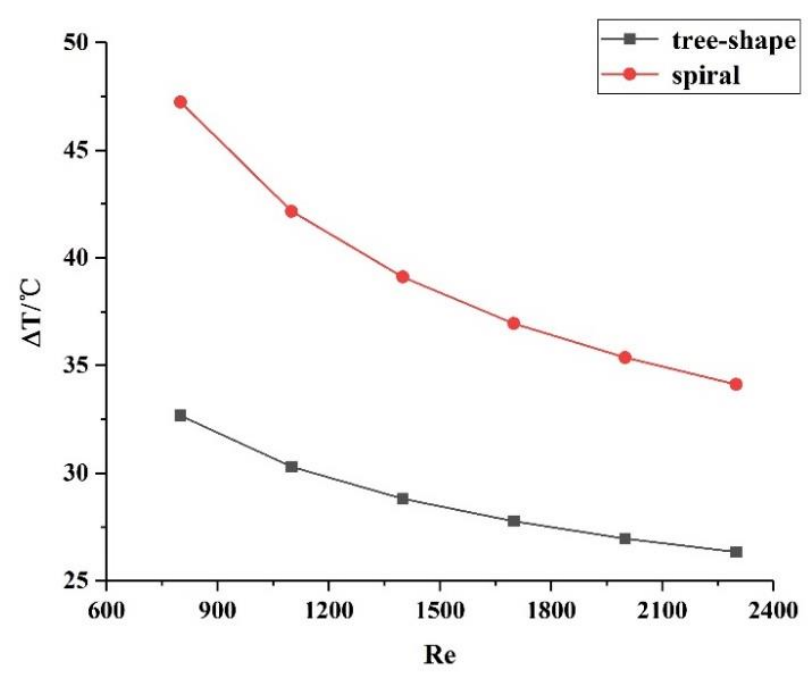

Figure 12 Comparison of temperature gradient between tree-shaped thermal exchanger and spiral thermal exchanger

From the average temperature of thermal exchanger (Figure 13), the average temperature of the tree-shaped microchannel network thermal exchanger is significantly lower than that of the conventional spiral thermal exchanger under the different Reynolds numbers, which shows high-speed motorized spindle using the tree-shaped microchannel network thermal exchanger can have higher processing accuracy under the same processing conditions.

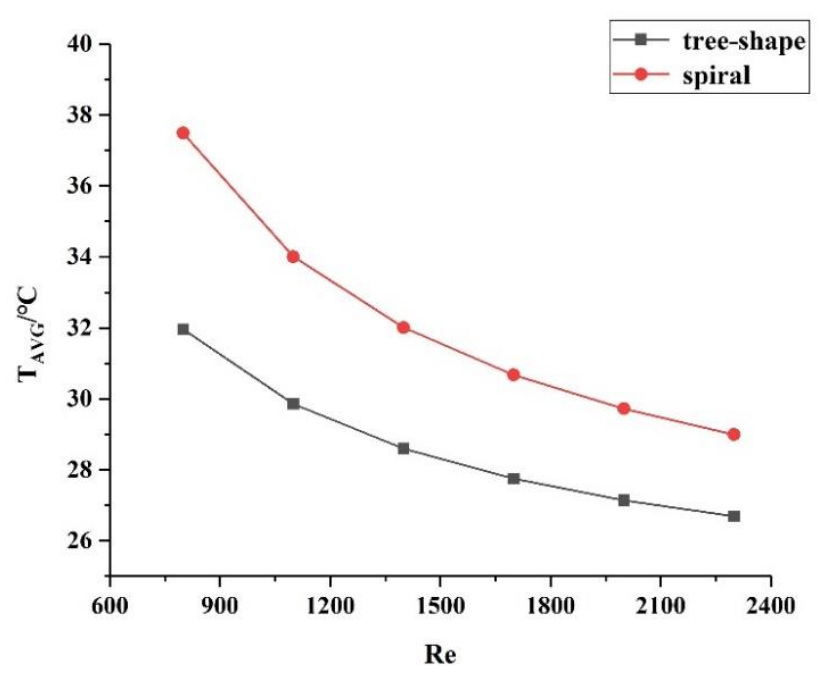

Figure 13 Comparison of average temperature between 
tree-shaped thermal exchanger and spiral thermal exchanger

\subsection{Hydrodynamic characteristics}

\subsubsection{Laminar mixing}

The cooling water dynamic characteristics was analyzed when $R e=2300$, which is seen from Figure 14(a). In the afflux channel, the diffluence flow of the cooling water (from $k$-th to $(k+1)$-th channel level) will form a radial vortex at the inner corner (Figure 14.a-a). The cooling water with a higher flow rate on the outer wall is continuously transported into the inner corner and mixed with the liquid near the inner corner wall for sufficient heat exchange. The flow velocity decreases at each branch. At the efflux channel in Figure 14(b), the confluence flow (from $(k+1)$-th to $k$-th channel level) increases the cooling water flow rate. And the radial vortex and axial vortex formed near the wall of the confluence (Figure 14.b-b). The cooling water has sufficient heat exchange. Flow out of the thermal exchanger at a higher flow rate. The cooling water through a complete tree-shaped microchannel network has experienced the process of diffluence flow deceleration and confluence flow acceleration.

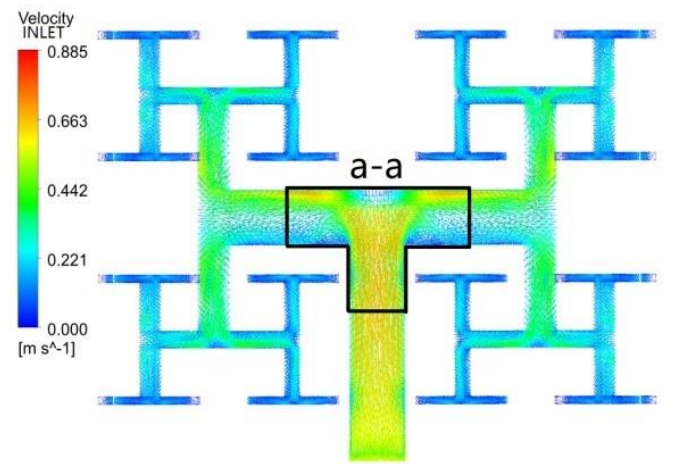

(a)

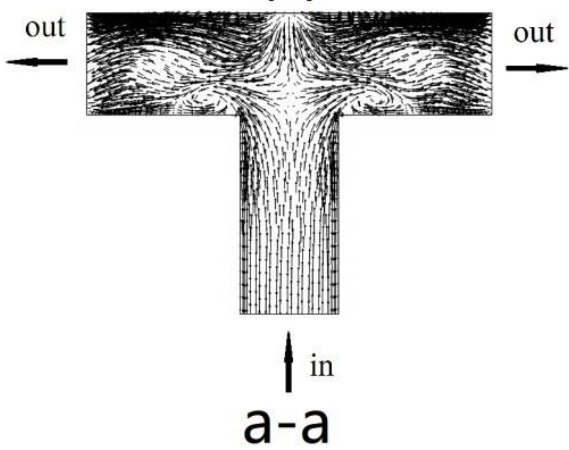

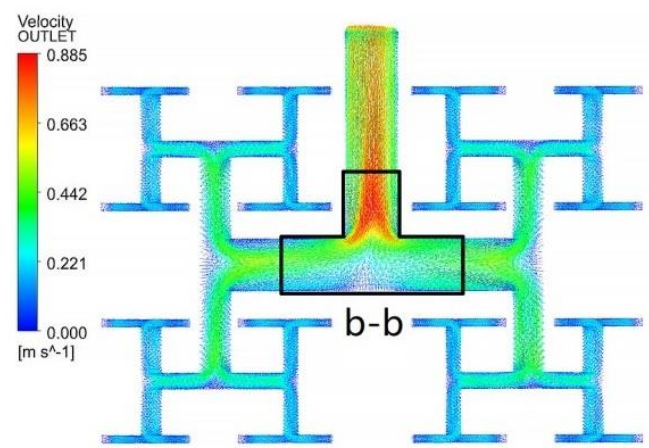

(b)

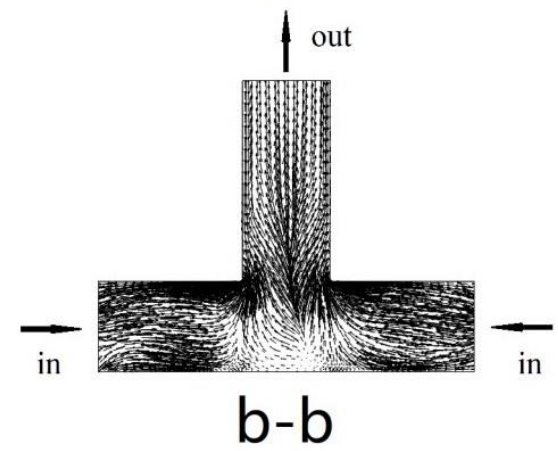

Figure 14 Laminar mixing in tree-shaped thermal exchanger (a) Flow condition of tree-shaped thermal exchanger at $R=59 \mathrm{~mm}$ (b) Flow condition of tree-shaped thermal exchanger at $R=71 \mathrm{~mm}$ a-a. The vortex at the inner corner of the afflux channel $\mathbf{b}-\mathbf{b}$. The vortex of the efflux channel

\subsubsection{Pressure drop}

Pressure drop, one of the important indicators to evaluate the performance of the thermal exchanger, is used to measure how much pumping power is needed to flow through a complete waterway. The pressure drop is expressed by equation (13):

$$
\Delta p=p_{\text {in }}-p_{\text {out }},
$$

Where $\Delta p$ is the pressure drop, $p_{\text {in }}$ is the afflux channel's pressure, $p_{\text {out }}$ is the efflux channel's pressure. The pressure drop comparison between the tree-shaped microchannel network thermal exchanger and the conventional spiral thermal exchanger is shown in Figure 15. This shows the tree-shaped microchannel network thermal exchanger's pressure drop is smaller than the conventional spiral thermal exchanger. The tree-shaped thermal exchanger requires less pumping power. 


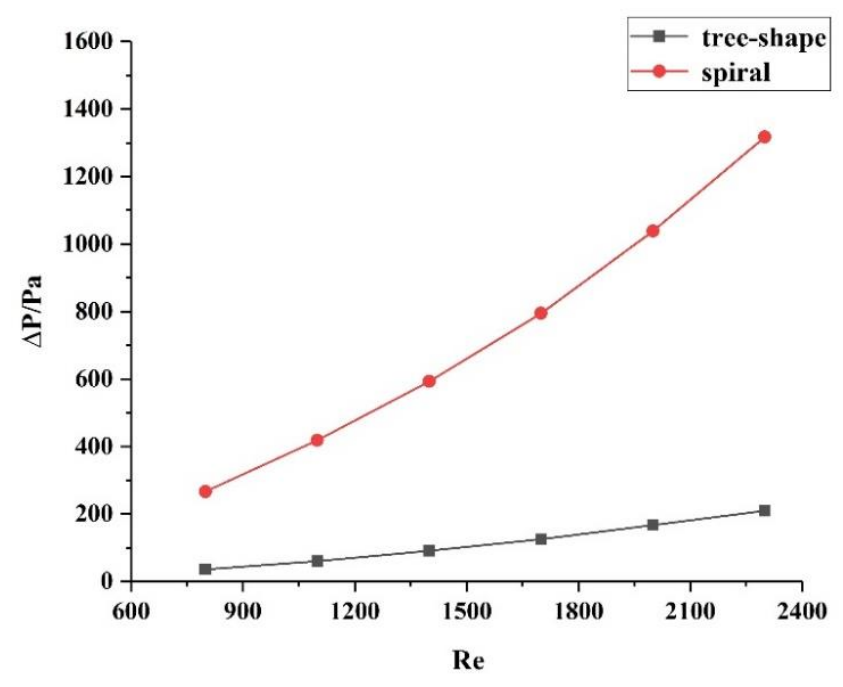

Figure 15 Comparison of pressure drop between tree-shaped thermal exchanger and spiral thermal exchanger

\subsection{Evaluation of heat transfer efficiency}

The COP is another index to measure the cooling performance of the thermal exchanger. It is expressed as the specific value of total heat transfer rate to fluid pumping power under constant temperature conditions. As equation (14):

$$
C O P=\frac{Q}{q_{v} \Delta p}
$$

Figure 16 shows the performance coefficient comparison boss the tree-shaped microchannel network thermal exchanger and the conventional spiral thermal exchanger under different Reynolds numbers. The COP of conventional spiral thermal exchanger is basically one-fifth of the tree-shaped thermal exchanger. The tree-shaped microchannel network thermal exchanger has better heat exchange performance.

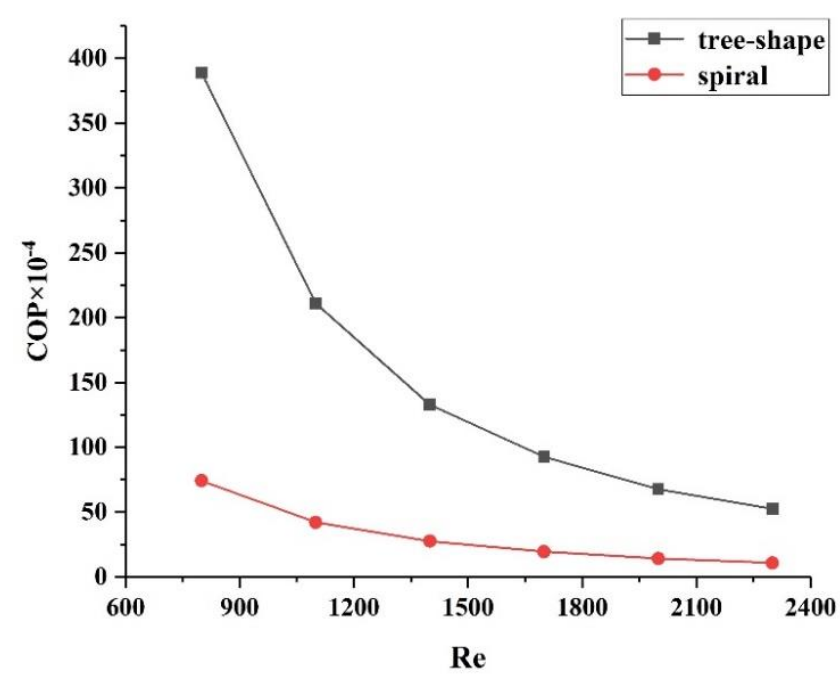

Figure 16 Comparison of performance coefficient between tree-shaped thermal exchanger and spiral thermal exchanger

\section{Conclusions}

(1) According to the construction theory of mammalian circulation and respiratory system, a tree-shaped microchannel network thermal exchanger is applied to the high-speed motorized spindle, which has practical application value.

(2) Considering the conjugate heat transfer between spindle stator, thermal exchanger and cooling water, a 3D fluid thermodynamic model is established. Both thermal exchangers have an identical heat exchange area and inlet size. The hydrodynamic characteristics, pressure loss, temperature gradient distribution and COP are compared.

(3) The numerical analysis results indicated that the new structure proposed in this paper has more vortices in the cooling water during the heat exchange process and can carry out sufficient heat exchange. The COP is fivefold that of a conventional spiral thermal exchanger, with less pumping power required, less temperature gradient changes and more uniform thermal field distribution.

(4) The relevant experimental results are consistent with the numerical analysis results, which verifies the theoretical analysis's correctness.

(5) The tree-shaped thermal exchanger structure designed in this paper can reduce the machining precision requirement and simplify the spindle structure. It can be used directly as the spindle shell and shorten the spindle production cycle and relatively improve the machining accuracy of spindle. Therefore it will have a broad application prospect in spindle heat transfer. 


\section{Declaration}

\section{Acknowledgements}

The authors sincerely thanks to the master candidate Chao Ma for his help on the numerical calculation equipment.

\section{Funding}

This research is supported by NSFC Grant Numbers 51975388 , Liaoning Natural Science Foundation 2019-MS-266. The Project is also sponsored by "Liaoning BaiQianWan Talents Program” .

\section{Availability of data and materials}

The datasets used and/or analysed during the current study are available from the corresponding author on reasonable request.

\section{Authors' contributions}

The author' contributions are as follows: $\mathrm{SH}$ was in charge of the whole trial; $\mathrm{ZC}$ wrote the manuscript; $\mathrm{YH}$ processed part of the data. WN embellished the content of the article. All authors read and approved the final manuscript.

\section{Competing interests}

The authors declare no competing financial interests.

\section{Consent for publication}

Not applicable

\section{Ethics approval and consent to participate}

Not applicable

\section{Nomenclature.}

$L_{k}=$ channel length, $\mathrm{mm}$

$\dot{L}_{k}^{k}=$ arc channel length, $m m$

$L_{\text {in/out }}=$ inlet/outlet channel length, $\mathrm{mm}$

$D_{k}=$ hydraulic diameter

$N=$ channel branches number

$D=$ channel length distribution size

$\Delta=$ channel diameter size

$W_{k}=$ branch channel width of the $\mathrm{k}$-th branch layer, $\mathrm{mm}$

$h=$ channel depth, $m m$

$L=$ thermal exchanger length, $m m$

$R_{m}=$ thermal exchanger inner and outer radius, $\mathrm{mm}$

$r_{m}=$ channel inner and outer radius, $\mathrm{mm}$

$S_{m}=$ the length of each segment, $\mathrm{mm}$

$\theta_{k}=$ the central angle corresponding to the sector channel, ${ }^{\circ}$

$\omega_{k}=\dot{L}_{k}$ corresponding central angle, ${ }^{\circ}$

$u, v, w=$ velocity components in $\mathrm{x}, \mathrm{y}, \mathrm{z}$ directions, $m / s$

$\rho=$ density, $\mathrm{kg} / \mathrm{m}^{3}$

$c_{p}=$ specific heat capacity, $J /(\mathrm{kg} \cdot \mathrm{K})$

$Q=$ total thermal transfer rate of fluid flow

$q_{v}=$ volume flow rate

$\lambda=$ Thermal Conductivity, $W /(m \cdot K)$

$T=$ temperature, $K$

$p=$ pressure, $P$

\section{Subscript.}

$k=$ number of branches

$m=$ positive integer

in =inlet

out $=$ outlet

$f=$ fluid

$s=$ solid

$\Gamma=$ channel surface

$A V G=$ average

\section{References}

[1] Josef, M., Jerzy, J., Eckart, U., M., Alkan, D., Wolfgang, K., Frank, H., Klaus, W., Toshimichi, M., Paul, S., Robert, S., Christian, B., Timo, W., \& Konrad, W. (2012). Thermal issues in machine tools. CIRP Annals, 61(2), 771-791.

[2] Jedrzejewski, J., Kwasny, W., Kowal, Z., \& Modrzycki, W. (2008). Precise model of HSC Machining Centre for aerospace parts machining. https://www.researchgate.net/publication/265082390_PRECISE_M ODEL_OF_HSC_MACHINING_CENTRE_FOR_AEROSPACE_P ARTS_MACHINING.pdf.

[3] Bryan, J.B., Donaldson, R.R., Clouser, R.W., \& Blewett, W.H. (1973). Reduction of Machine Tool Spindle Growth. U.S. Department of Energy Office of Scientific and Technical Information. Technical Report. https://www.osti.gov/biblio/4479736. Published 29 March 1974.

[4] Bryan, J.B., Carter, D.L., Clouser, R.W., \& Hamilton, J.H. (1982). Order of Magnitude Improvement in Thermal Stability with use of Liquid Shower on a general purpose Measuring Machine. U.S. Department of Energy Office of Scientific and Technical Information. Conference. https://www.osti.gov/biblio/5384541. Published 6 May 1982.

[5] Donmez, M.A., Hahn, M.H., \& Soons, J.A. (2007). A novel cooling system to reduce thermally-induced errors of machine tools. CIRP Annals, 56(1), 521-524.

[6] Bejan A. (1997). Constructal-theory network of conducting paths for cooling a heat generating volume. International Journal of Heat and Mass Transfer, 799-816.

[7] Bejan, A., Errera, M.R. (1997). Deterministic tree networks for fluid flow: geometry for minimal flow resistance between a volume and one point. Fractals, 5(4), 685-695.

[8] Bejan, A., \& Lorente, S. (2004). The constructal law and the thermodynamics of flow systems with configuration. Int $\mathrm{J}$ Heat Mass Transfer, 47(14-16), 3203-3214. 
[9] Bejan, A., \& Lorente, S. (2006). Constructal theory of generation of configuration in nature and engineering. Journal of Applied Physics, 100(4), 1-27.

[10] Wechsatol, W., Lorente, S., \& Bejan, A. (2002). Optimal tree-shaped networks for fluid flow in a disc-shaped body. Int $\mathrm{J}$ Heat Mass Transfer, 45(25), 4911-4924.

[11] Gosselin, L. (2007). Optimization of tree-shaped fluid networks with size limitations. International Journal of Thermal Science, 46(5), 434-443.

[12] Chen, Y.P., \& Cheng, P. (2002). Heat transfer and pressure drop in fractal tree-like microchannel nets. International Journal Heat and Mass Transfer, 45(13), 2643-2648.

[13] Chen, Y., \& Cheng, P. (2005). An experimental investigation on the thermal efficiency of fractal tree-like microchannel nets. International Communications in Heat and Mass Transfer, 32(7), 931-938.

[14] Senn, S.M., \& Poulikakos, D. (2004). Laminar mixing, heat transfer and pressure drop in tree-like microchannel nets and their application for thermal management in polymer electrolyte fuel cells. Journal of Power Sources, 130(1-2), 178-191.

[15] Senn, S.M., \& Poulikakos, D. (2006). Pyramidal direct methanol fuel cells. International Journal of Heat and Mass Transfer, 49(7-8), 1516-1528.

[16] Alharbi, A.Y., Pence, D.V., \& Cullion, R.N. (2003). Fluid Flow Through Microscale Fractal-Like Branching Channel Networks. The American Society of Mechanical Engineering, Journal of Fluids Engineering, 125(6), 1051-1057.

[17] Pence, D.V. (2002). Reduced pumping power and wall temperature in microchannel heat sinks with fractal-like branching channel networks. Microscale Thermophysical Engineering, 6(4), 319-330.

[18] Jing, D., Song, S., Pan, Y. \& Wang, X. (2018). Optimal fractal tree-like microchannel networks with slip for laminar-flow-modified Murray's law, Beilstein Journal of Nanotechnology, 9, 482-489.

[19] Jing, D., He, L., \& Wang, X. (2018). Optimization analysis of fractal tree-like microchannel network for electroviscous flow to realize minimum hydraulic resistance, International Journal of Heat and Mass Transfer, 125, 749-755.

[20] Jing, D., Yi, \& S.S. (2019). Electroosmotic Flow in Tree-like Branching Microchannel Network. Fractals, 27(6). doi:10.1142/S02 $18348 X 19500956$.

[21] Xia, C.H., Fu, J.Z., Lai, J.T., Yao, X.H., \& Chen, Z.C. (2015). Conjugate heat transfer in fractal tree-like channels network heat sink for high-speed motorized spindle cooling. Applied Thermal Engineering, 90, 1032-1042.

[22] Wang, X.Q., Mujumdar, A.S., \& Yap, C. (2006). Numerical analysis of blockage and optimization of heat transfer performance of fractal-like microchannel nets. Journal Electronic Packaging, 128(1), $38-45$.
[23] Mandelbrot, B.B. (1983). The fractal geometry of nature/ B.B. Mandelbrot. Am. J. Phys. 51(3), 286-286. doi: 10.1119/1.13295.

[24] Murray, C.D. (1926). The physiological principle of minimum work. I. The vascular system and the cost of blood volume. Proc Natl Acad Sci USA. 12(3), 207-214. doi: 10.1073/pnas.12.3.207.

[25] Bejan, A., Rocha, LAO., \& Lorente S. (2000). Thermodynamic optimization of geometry: T-and Y-shaped constructs of fluid streams. International Journal of Thermal Sciences, 39(9-11), 949960.

\section{Biographical notes}

Song-Hua Li, born in 1977 , is currently a professor and a $\mathrm{PhD}$ candidate supervisor at National-Local Joint Engineering Laboratory of NC Machining Equipment and Technology of High-Grade Stone, Shenyang Jianzhu University, China. His research interests include high performance $\mathrm{CNC}$ machine tool spindle system, high efficiency and precision machining technology and equipment for intractable materials, high-grade stone CNC machining equipment and technology and modern construction engineering equipment and technology.

Tel: +86-024-24690319; E-mail: lisonghua@sjzu.edu.cn

Zi-Chen Zhao, born in 1995, is currently a master candidate at School of Mechanical Engineering, Shenyang Jianzhu University, China.

E-mail: syjzdxzzc@hotmail.com

Yong-Hua Wang, born in 1990, is currently a $\mathrm{PhD}$ candidate at School of Mechanical Engineering, Shenyang Jianzhu University, China.

E-mail: yonghua0514@163.com

Wei-Nan Wang, born in 1989 , is currently a $\mathrm{PhD}$ candidate at School of Mechanical Engineering, Shenyang Jianzhu University, China.

E-mail: robinwang@sjzu.edu.cn

\section{Appendix}

Appendix and supplement both mean material added at the end of a book. An appendix gives useful additional information, but even without it the rest of the book is complete: In the appendix are forty detailed charts. A supplement, bound in the book or published separately, is given for comparison, as an enhancement, to provide corrections, to present later information, and the like: A yearly supplement is issue. 
Table 4 Tree-shaped thermal exchanger structure parameters list

\begin{tabular}{ccccccc}
\hline \multicolumn{5}{c}{ Se $=800$} & \multicolumn{5}{c}{$\mathrm{Re}=2300$} \\
$P_{\text {in }}$ & 35.218 & 35.57 & -0.352 & 210.316 & 209.26 & 1.056 \\
$P_{\text {out }}$ & 0.124 & 0.14 & -0.016 & 0.832 & 0.86 & -0.037 \\
$T_{\text {in }}$ & 20 & 20 & 0 & 20 & 20 & 0 \\
$T_{\text {out }}$ & 32.231 & 29.490 & 2.741 & 26.128 & 23.910 & 2.218 \\
$T_{1}$ & 33.017 & 32.640 & 0.377 & 26.752 & 26.430 & 0.322 \\
$T_{2}$ & 33.426 & 32.840 & 0.586 & 27.574 & 27.090 & 0.484 \\
$T_{3}$ & 33.553 & 33.070 & 0.483 & 26.729 & 26.390 & 0.339 \\
$T_{4}$ & 26.766 & 26.440 & 0.326 & 22.633 & 22.390 & 0.243 \\
$T_{5}$ & 26.514 & 26.170 & 0.344 & 22.327 & 21.960 & 0.367 \\
$T_{6}$ & 28.442 & 28.360 & 0.082 & 23.131 & 22.700 & 0.431 \\
\hline
\end{tabular}

Table 5 Conventional spiral thermal exchanger structure parameters list

\begin{tabular}{lcccccc}
\hline & \multicolumn{5}{c}{ Re $=800$} & \multicolumn{3}{c}{ Re $=2300$} \\
\hline$P_{\text {in }}$ & 280.392 & 281.79 & -1.398 & 1418.828 & 1417.41 & 1.418 \\
$P_{\text {out }}$ & 14.367 & 14.22 & 0.147 & 101.869 & 102.38 & -0.511 \\
$T_{\text {in }}$ & 20 & 20 & 0 & 20 & 20 & 0 \\
$T_{\text {out }}$ & 45.293 & 42.350 & 2.943 & 31.123 & 28.990 & 2.133 \\
$T_{1}$ & 42.757 & 42.220 & 0.537 & 35.571 & 35.110 & 0.461 \\
$T_{2}$ & 48.682 & 47.810 & 0.972 & 37.866 & 37.410 & 0.456 \\
$T_{3}$ & 54.559 & 53.670 & 0.889 & 41.209 & 40.690 & 0.519 \\
$T_{4}$ & 27.033 & 26.590 & 0.443 & 22.503 & 22.220 & 0.283 \\
$T_{5}$ & 30.668 & 30.140 & 0.528 & 23.418 & 23.100 & 0.318 \\
$T_{6}$ & 36.052 & 35.450 & 0.602 & 25.536 & 25.230 & 0.306 \\
\hline
\end{tabular}


Figures

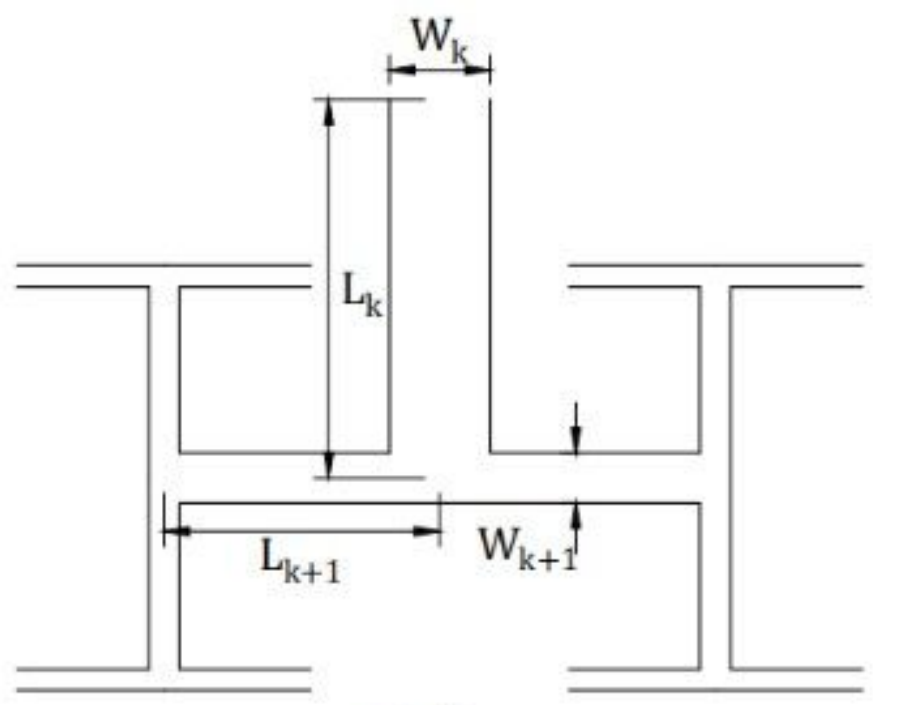

(a)

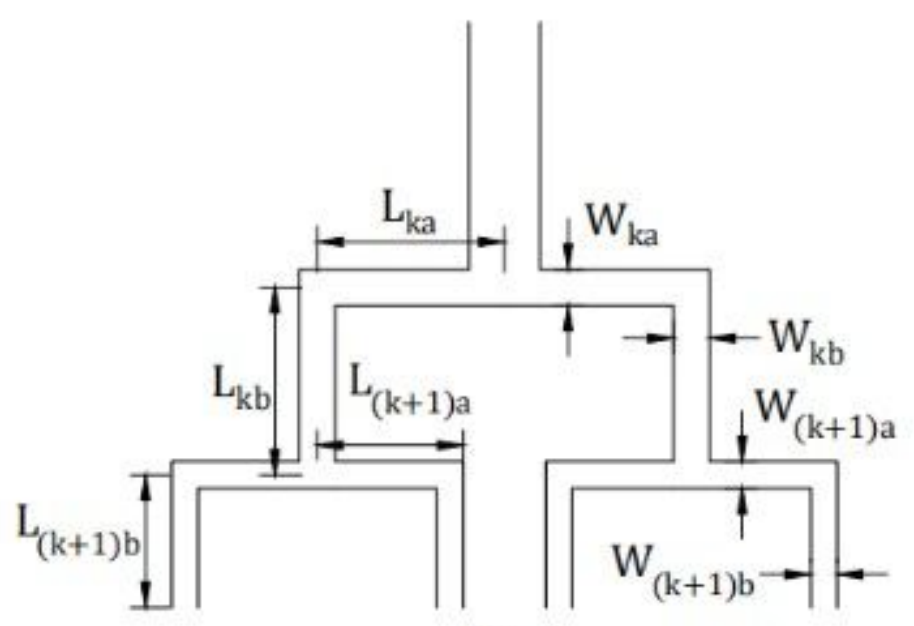

(b)

Figure 1

Two tree-shaped structures (a) The branch angle of $90^{\circ}(\mathrm{b})$ The branch angle of $180^{\circ}$

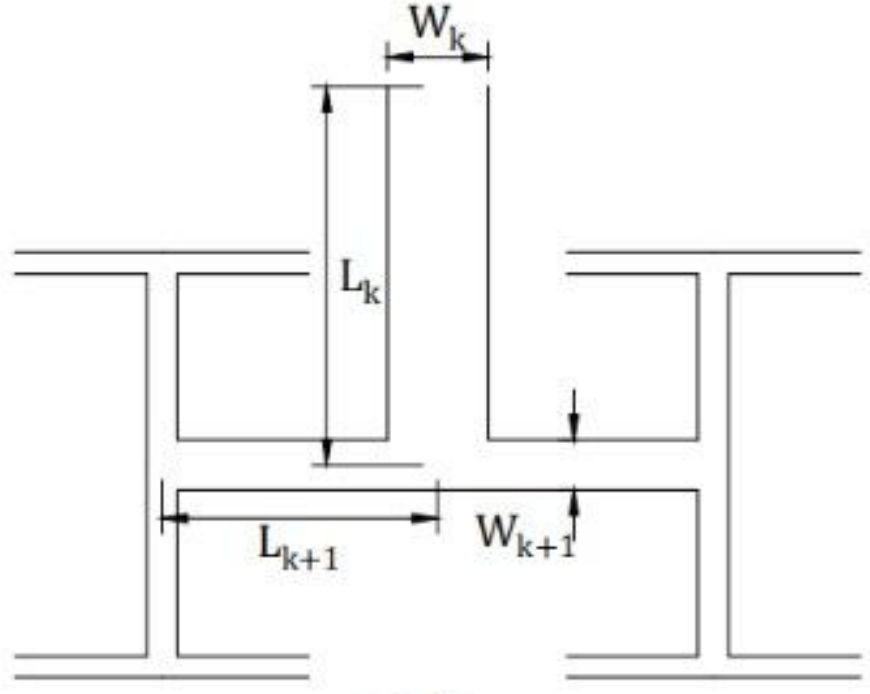

(a)

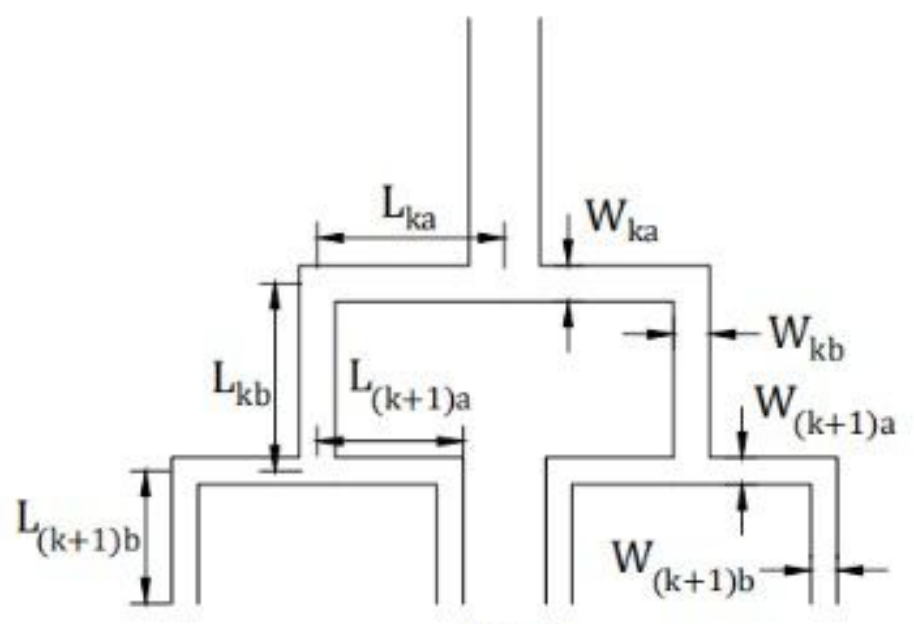

(b)

Figure 1

Two tree-shaped structures (a) The branch angle of $90^{\circ}$ (b) The branch angle of $180^{\circ}$ 


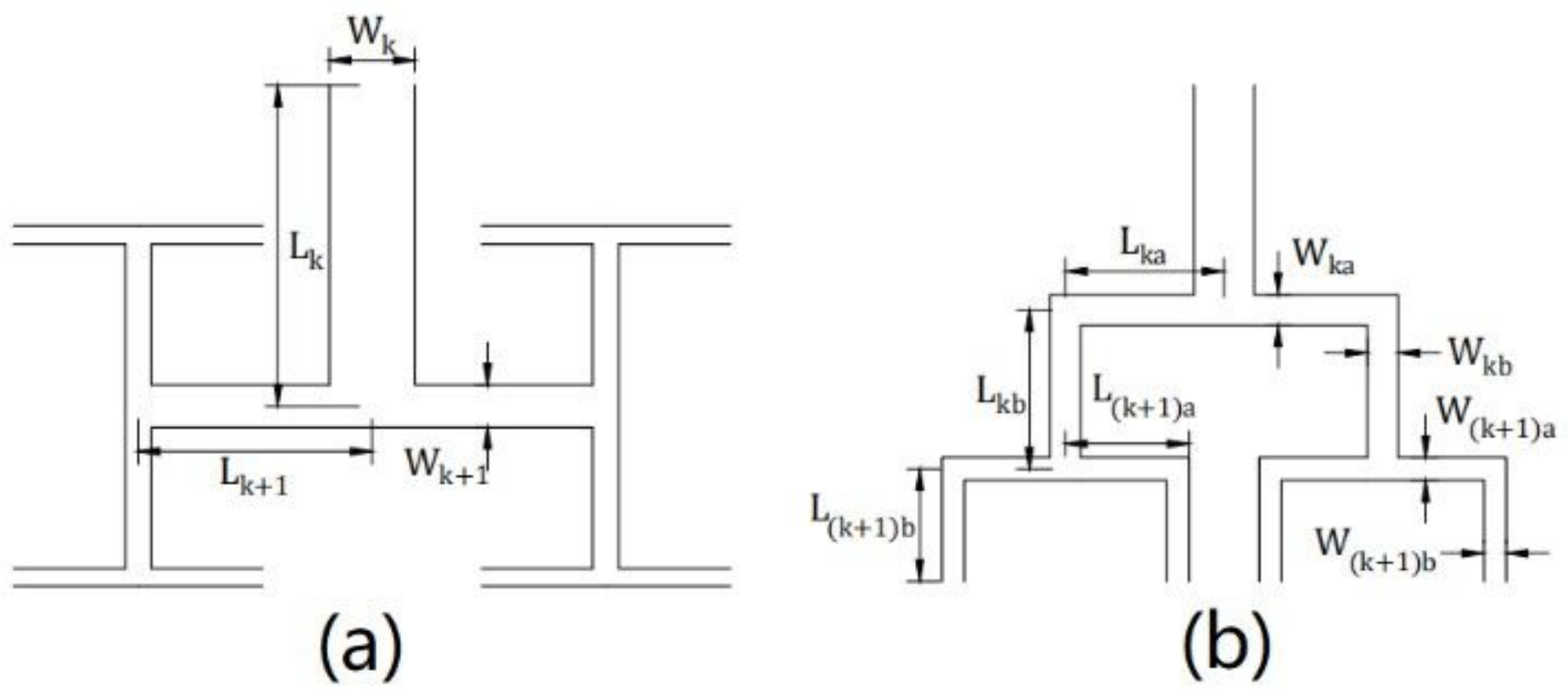

Figure 1

Two tree-shaped structures (a) The branch angle of $90^{\circ}$ (b) The branch angle of $180^{\circ}$ 


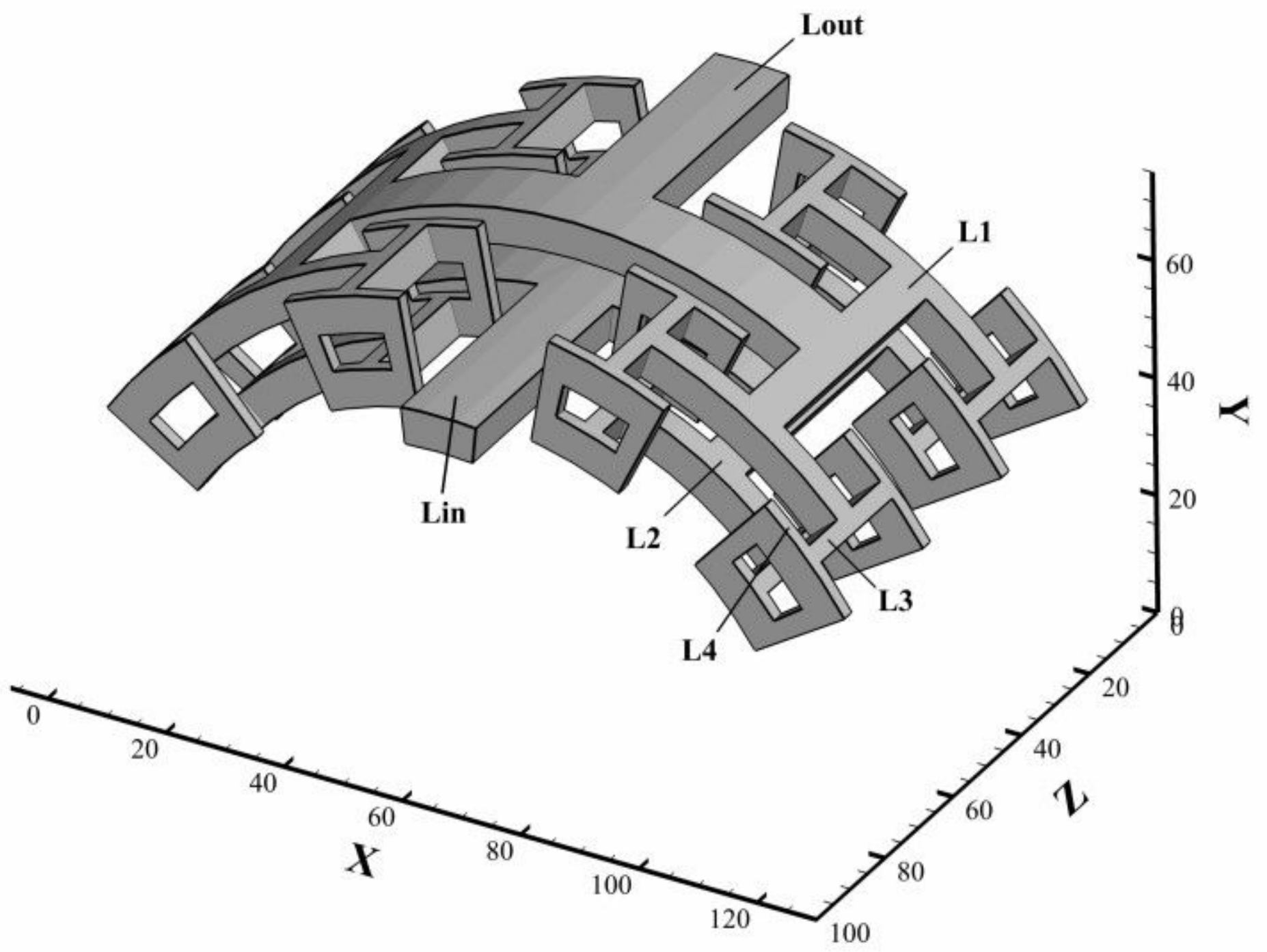

Figure 2

Cylindrical tree-shaped microchannel network structure 


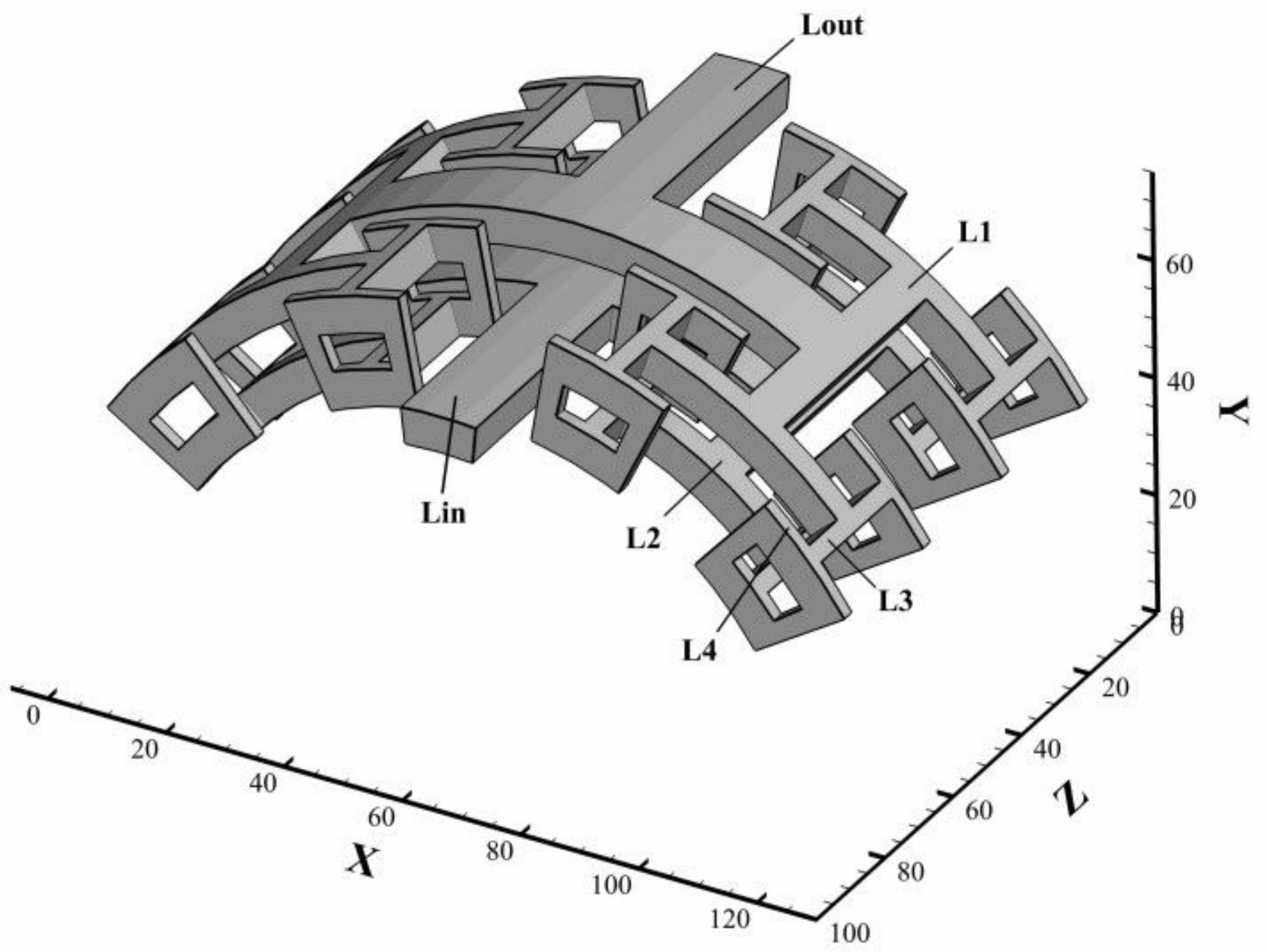

Figure 2

Cylindrical tree-shaped microchannel network structure 


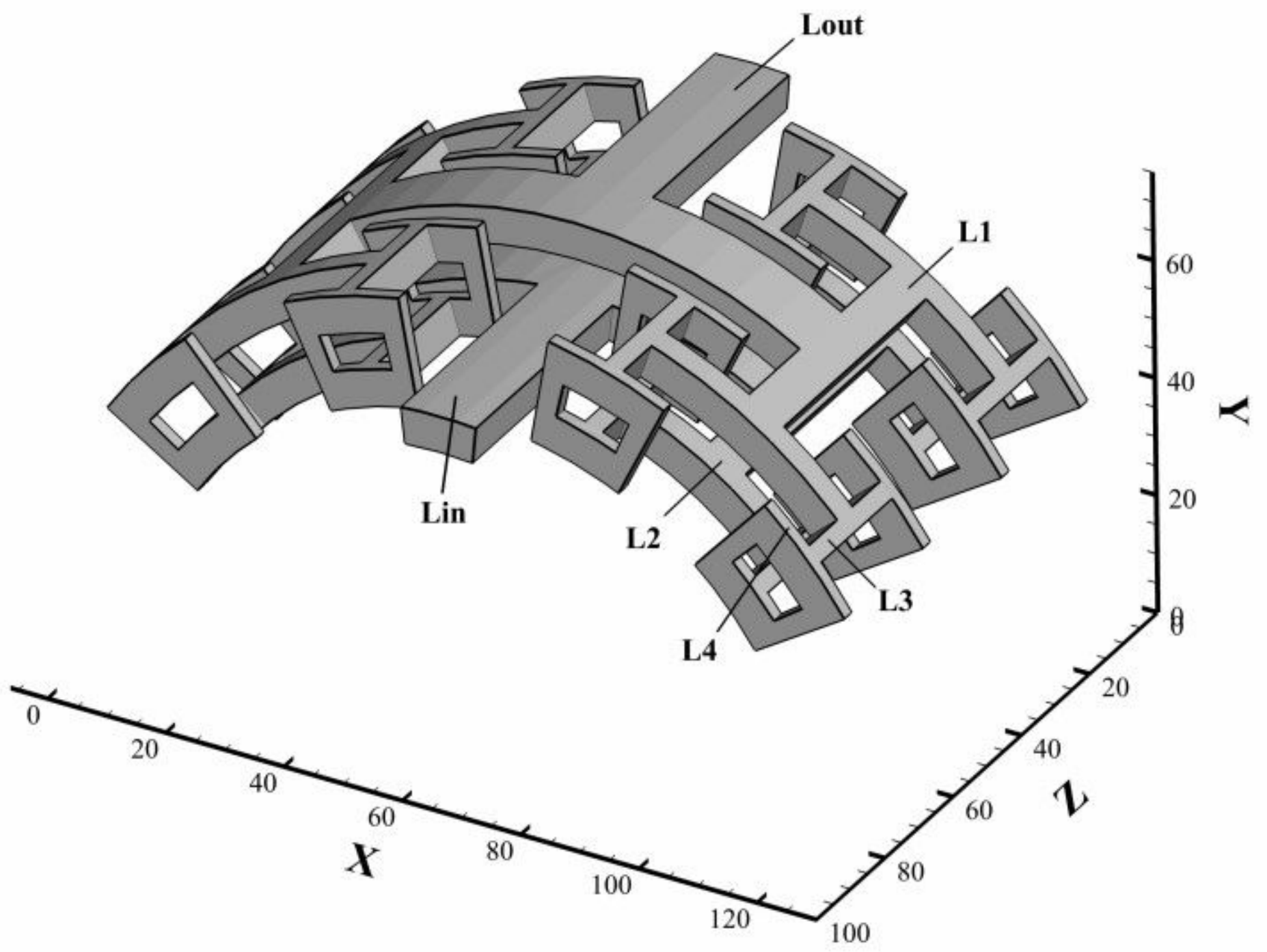

Figure 2

Cylindrical tree-shaped microchannel network structure 

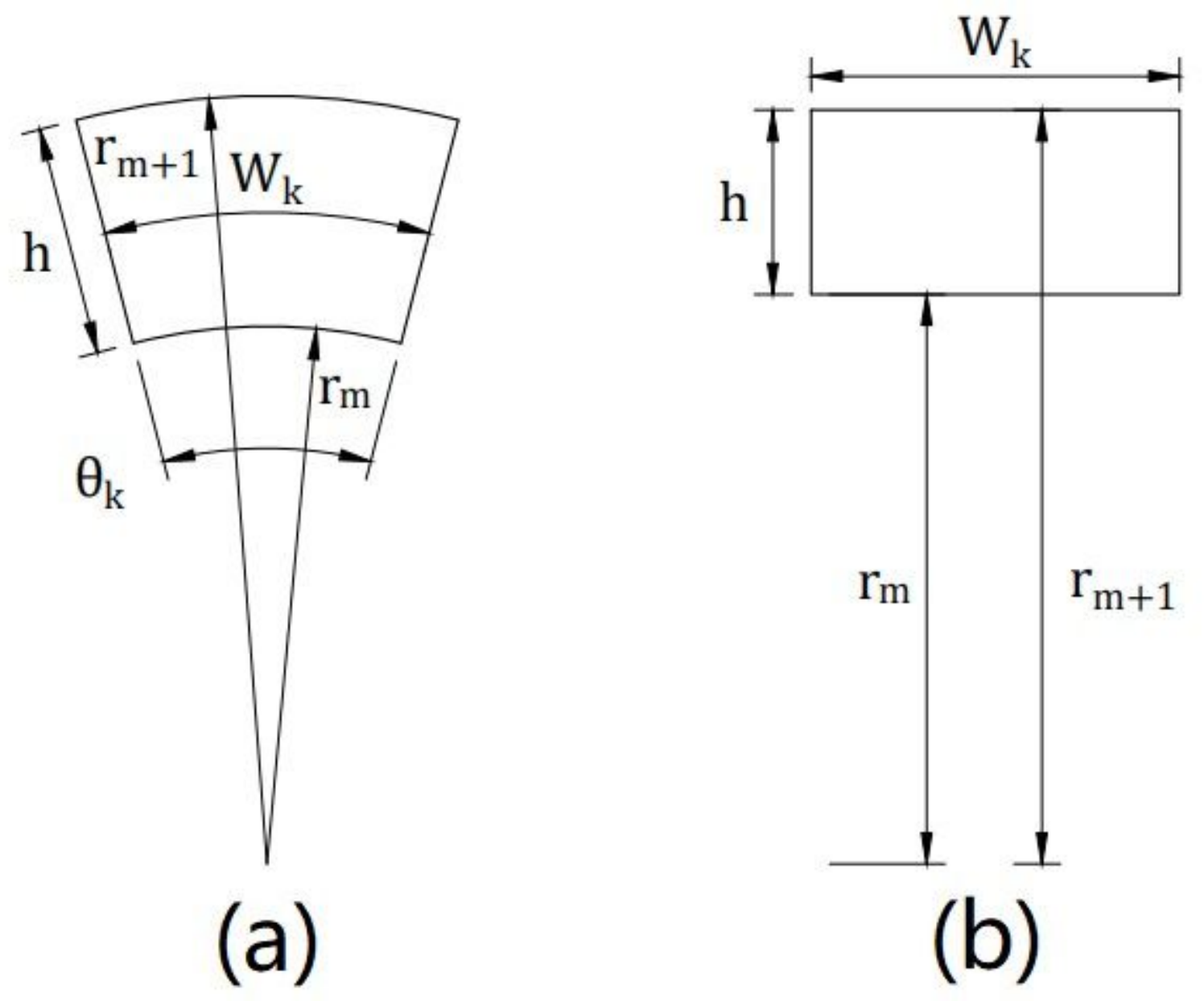

Figure 3

Lateral section shape (a) Axial channel lateral section (b) Circumferential channel lateral section 

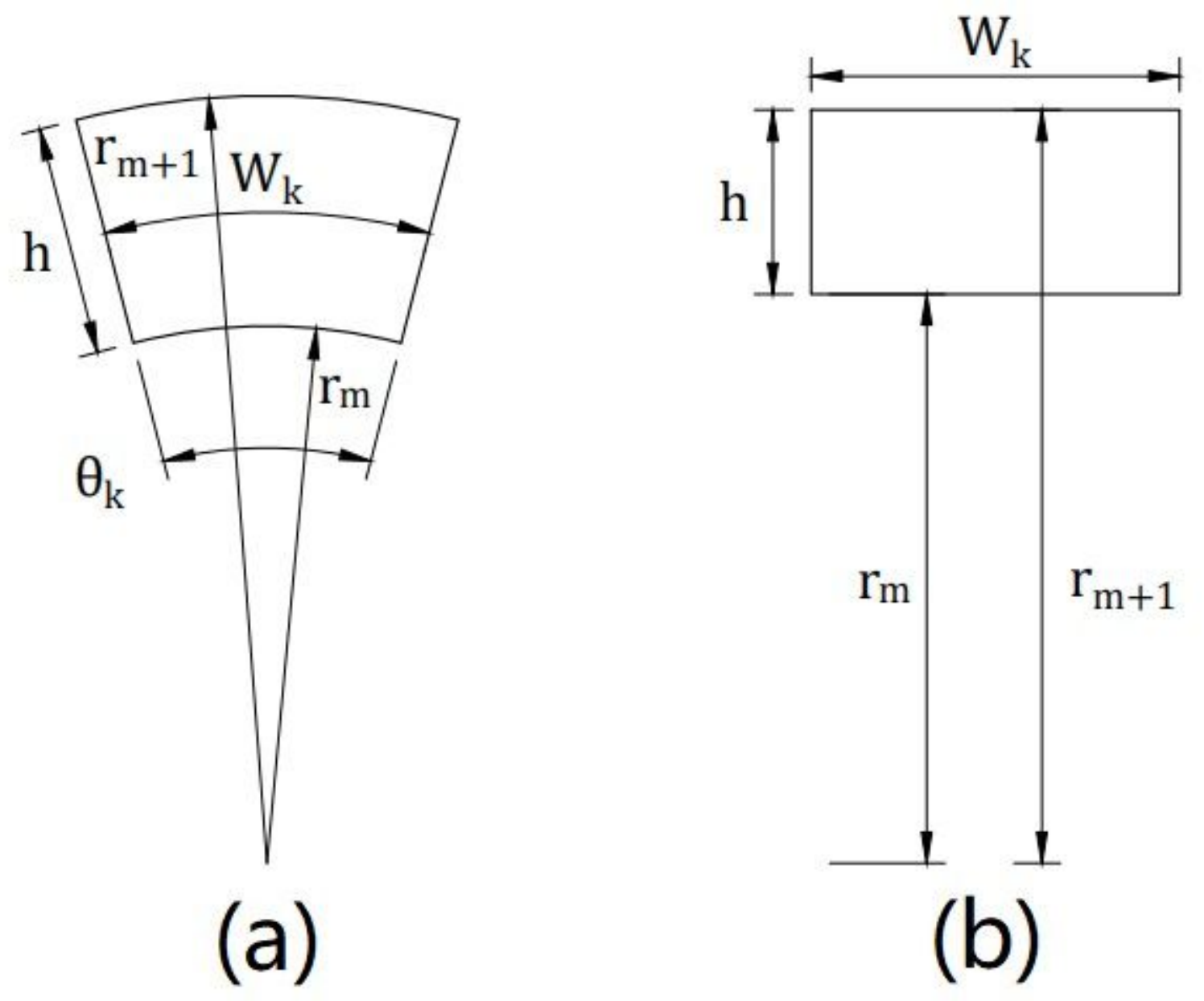

Figure 3

Lateral section shape (a) Axial channel lateral section (b) Circumferential channel lateral section 

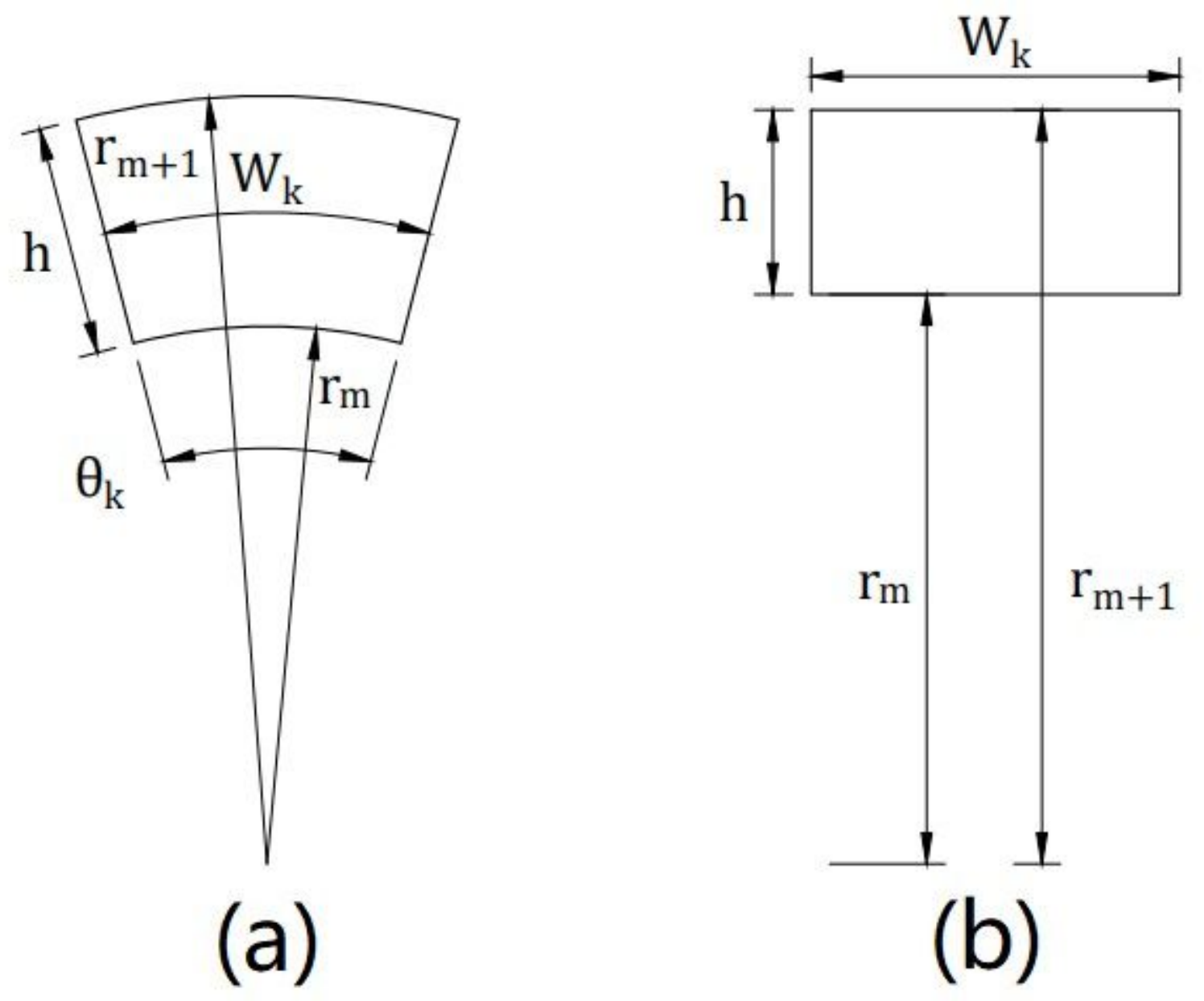

Figure 3

Lateral section shape (a) Axial channel lateral section (b) Circumferential channel lateral section 


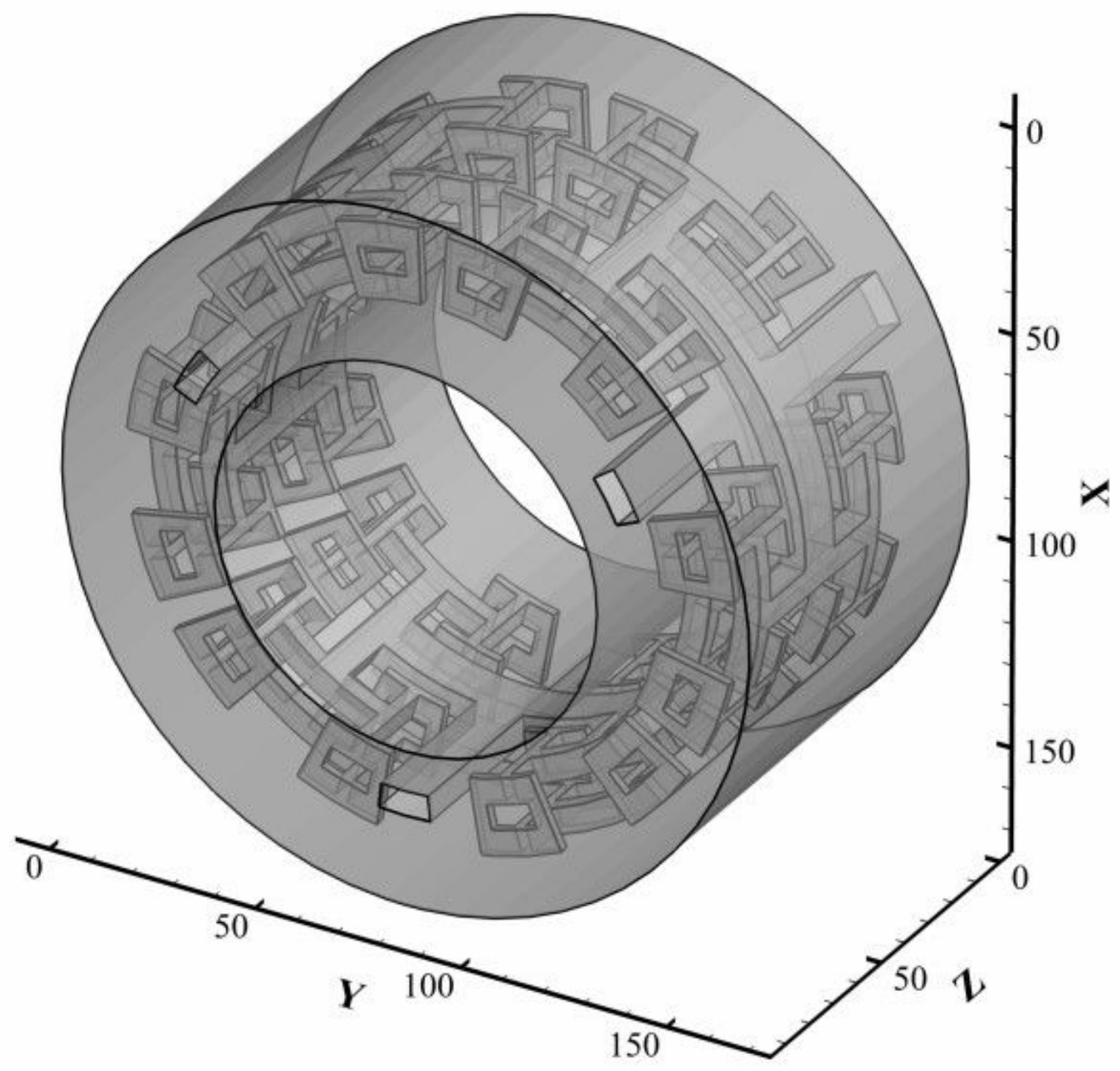

Figure 4

Cylindrical tree-shaped microchannel network thermal exchanger 


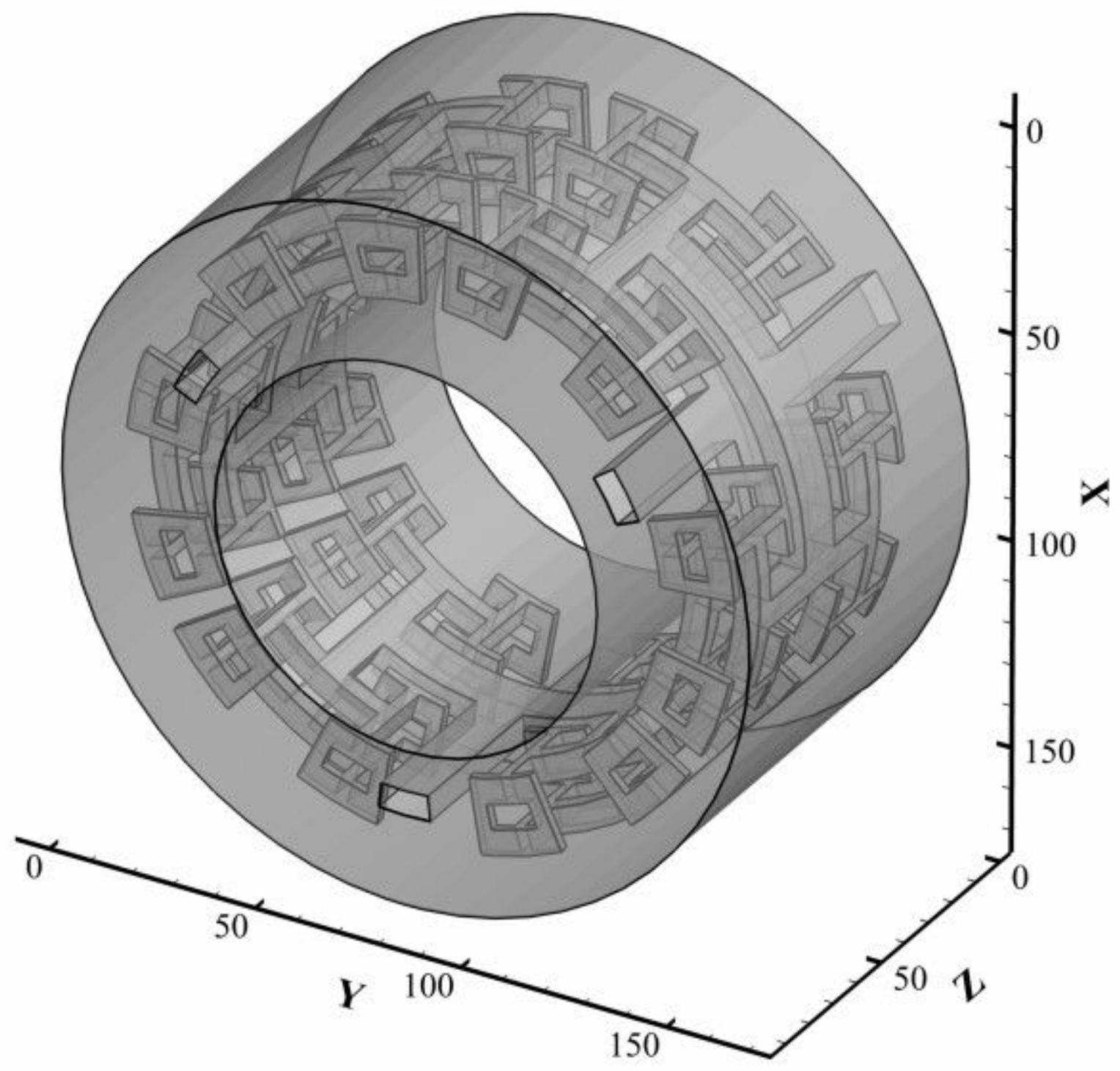

Figure 4

Cylindrical tree-shaped microchannel network thermal exchanger 


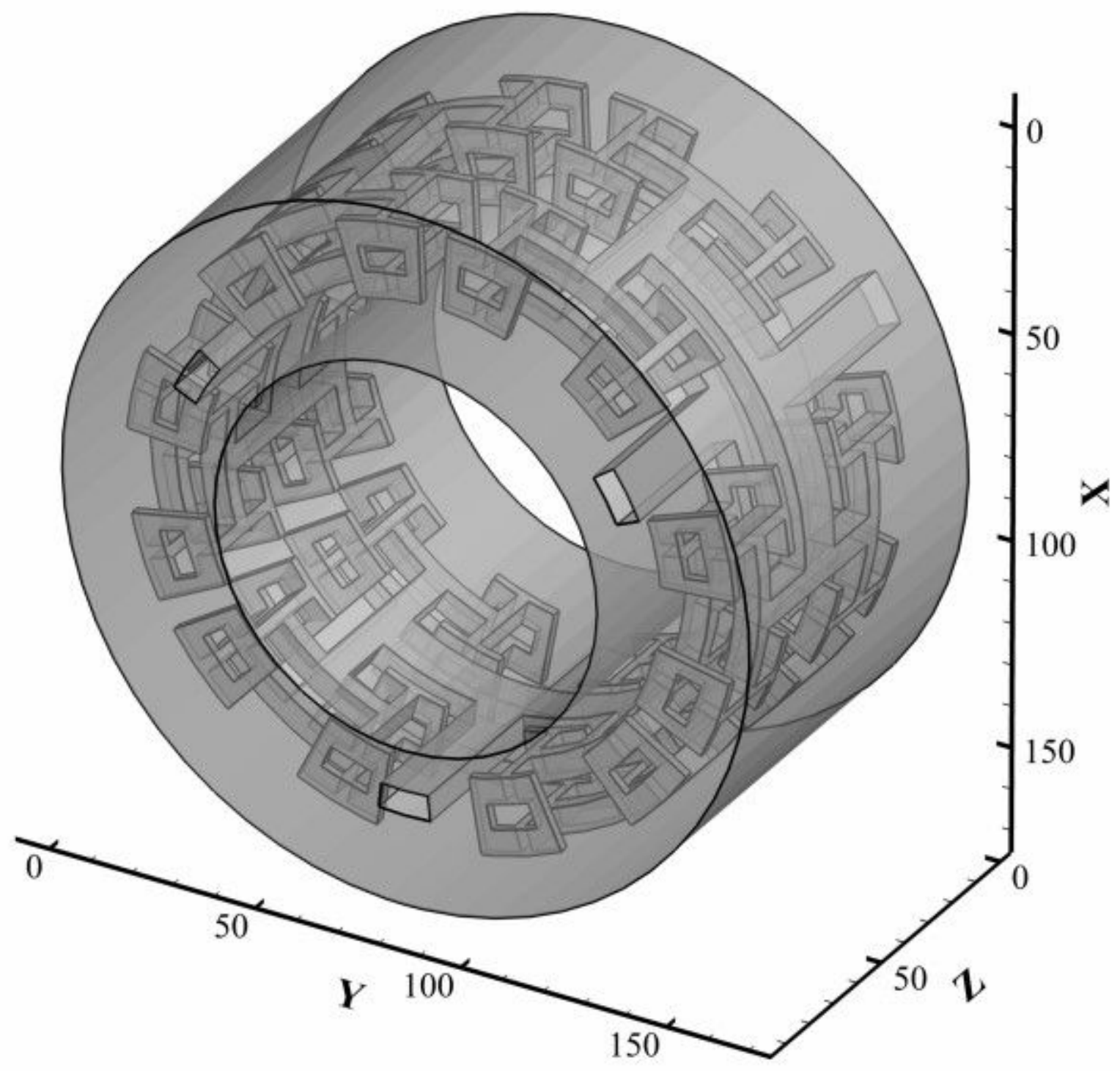

Figure 4

Cylindrical tree-shaped microchannel network thermal exchanger 


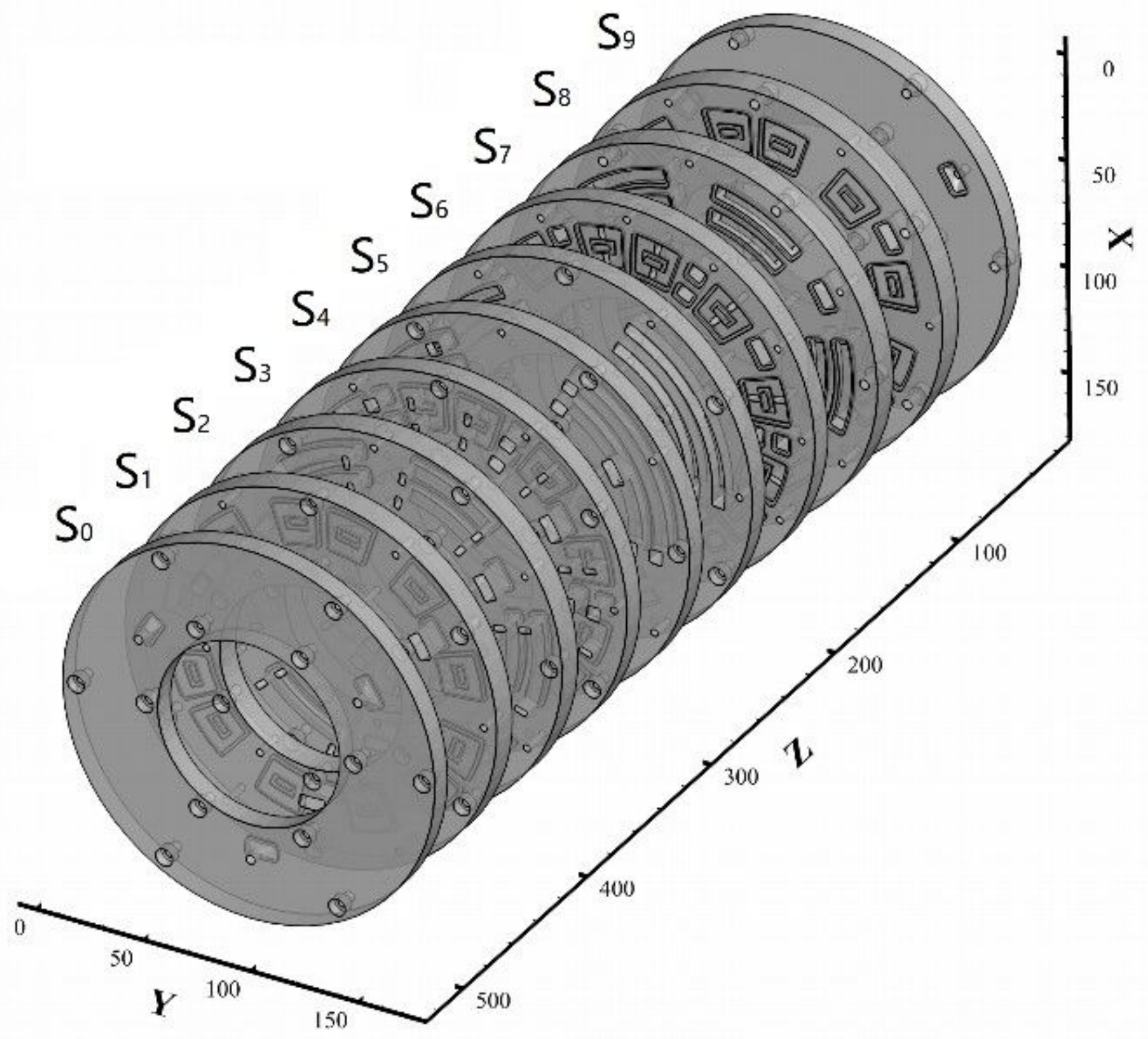

Figure 5

The segmented structure of tree-shaped microchannel network thermal exchanger 


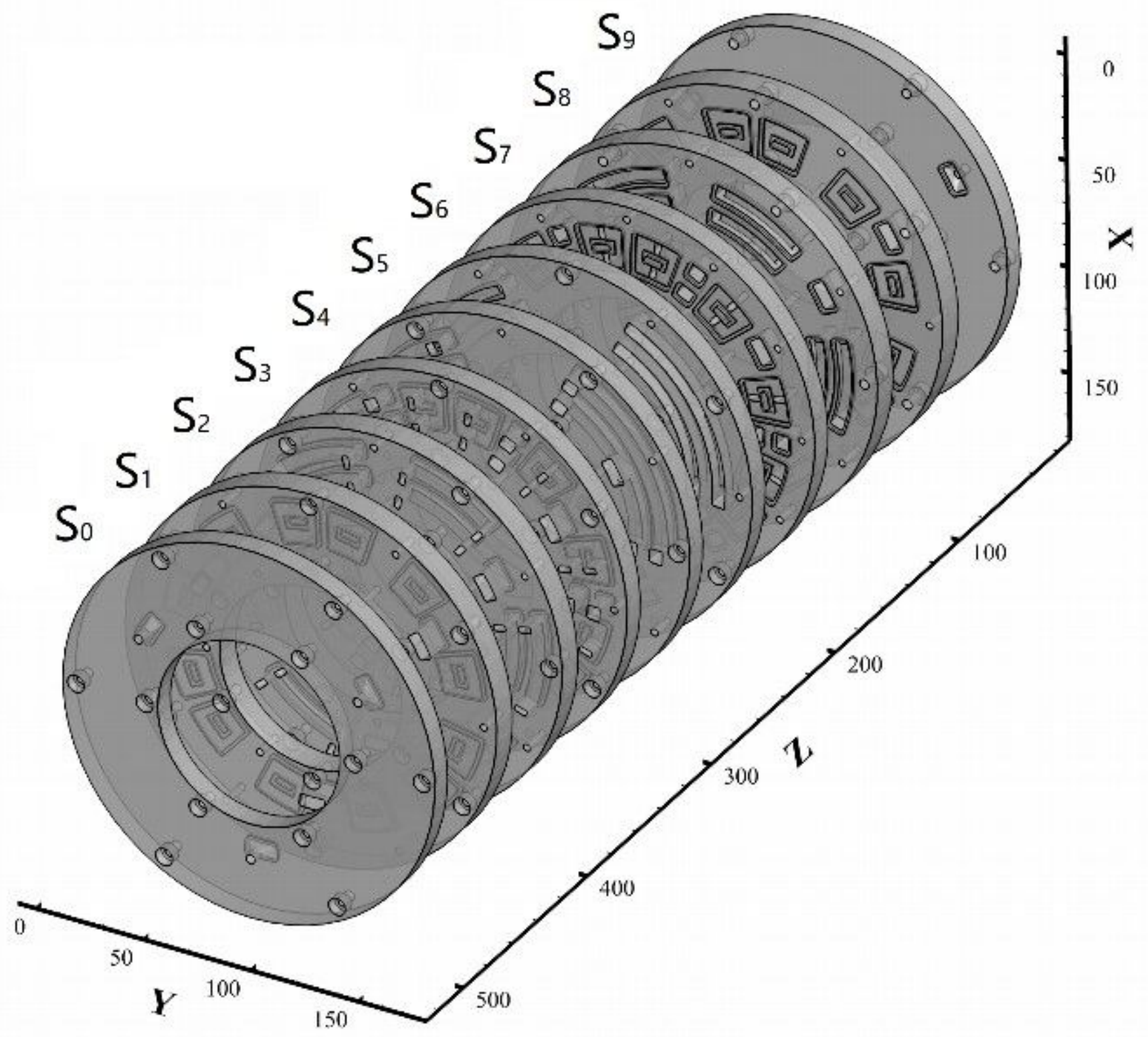

Figure 5

The segmented structure of tree-shaped microchannel network thermal exchanger 


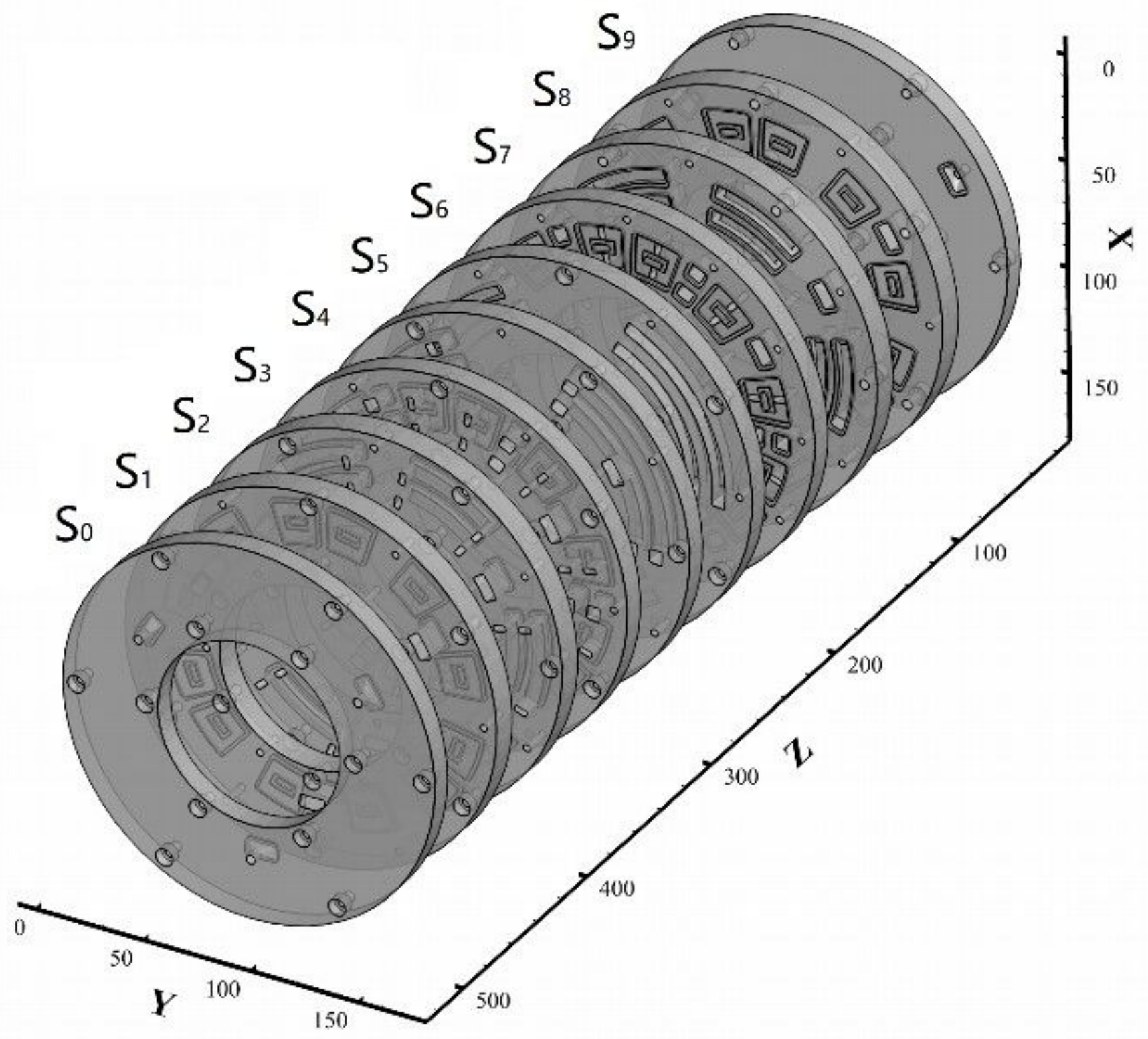

Figure 5

The segmented structure of tree-shaped microchannel network thermal exchanger 


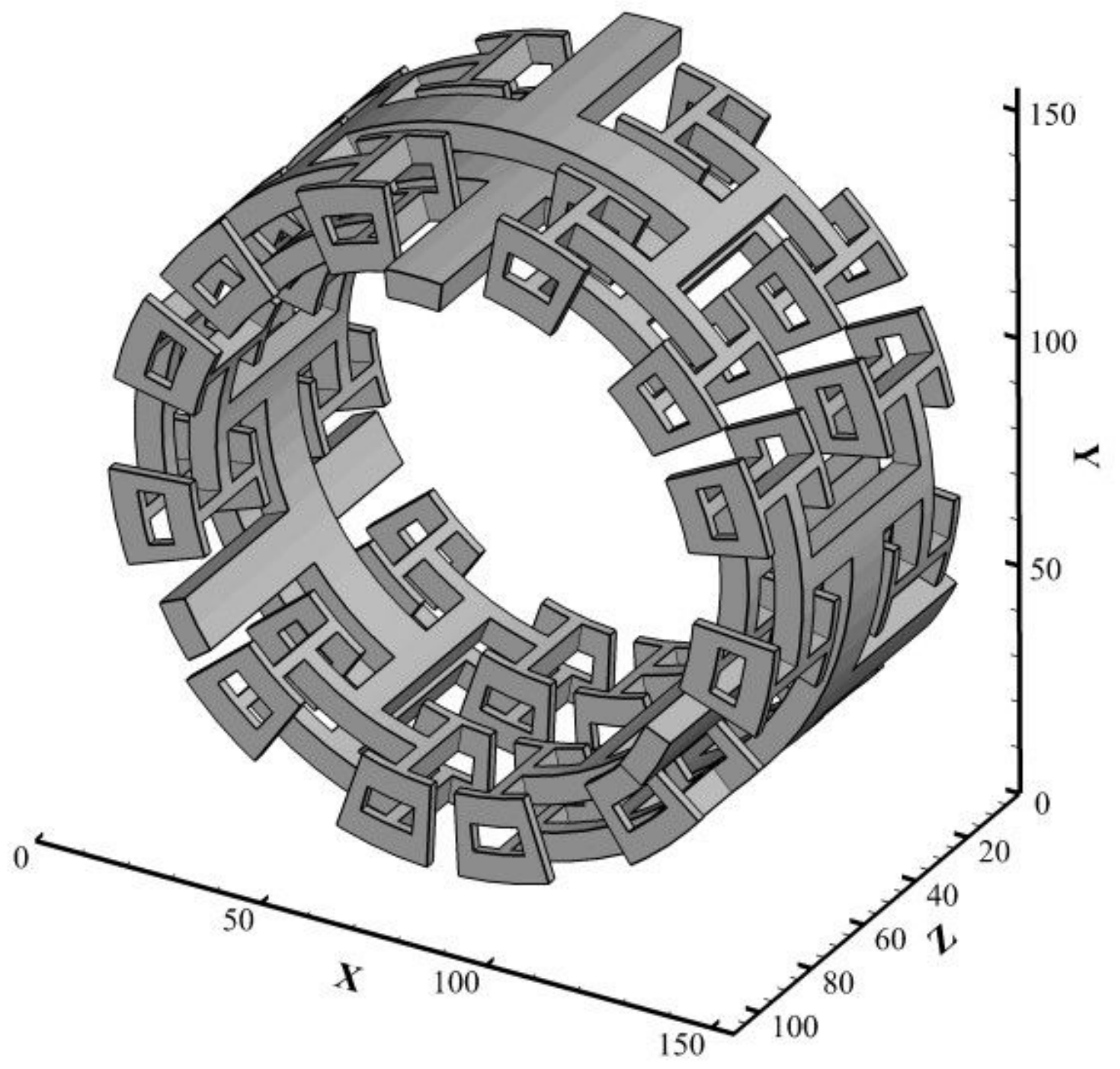

Figure 6

Tree-shaped microchannel network distribution map 


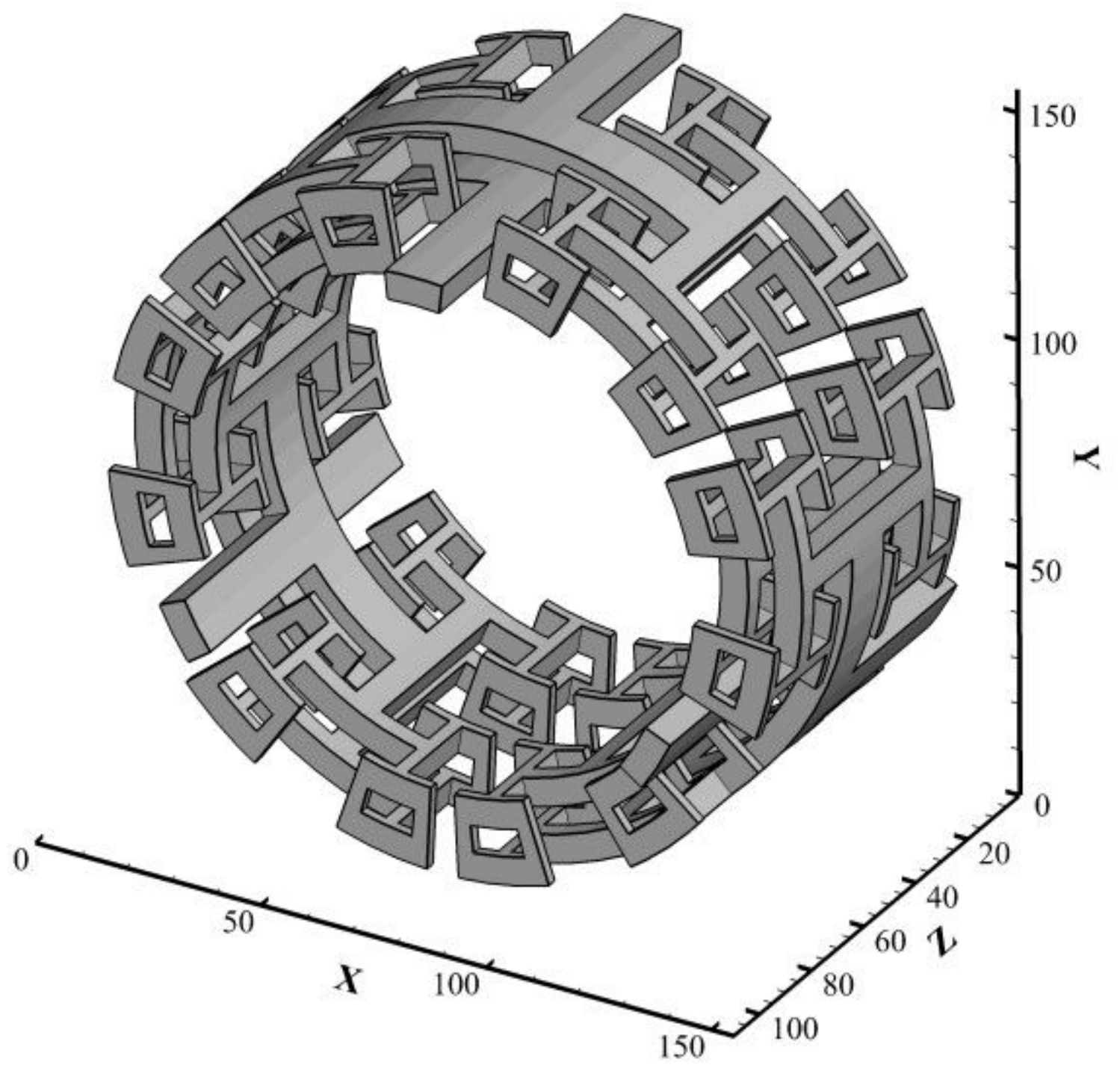

Figure 6

Tree-shaped microchannel network distribution map 


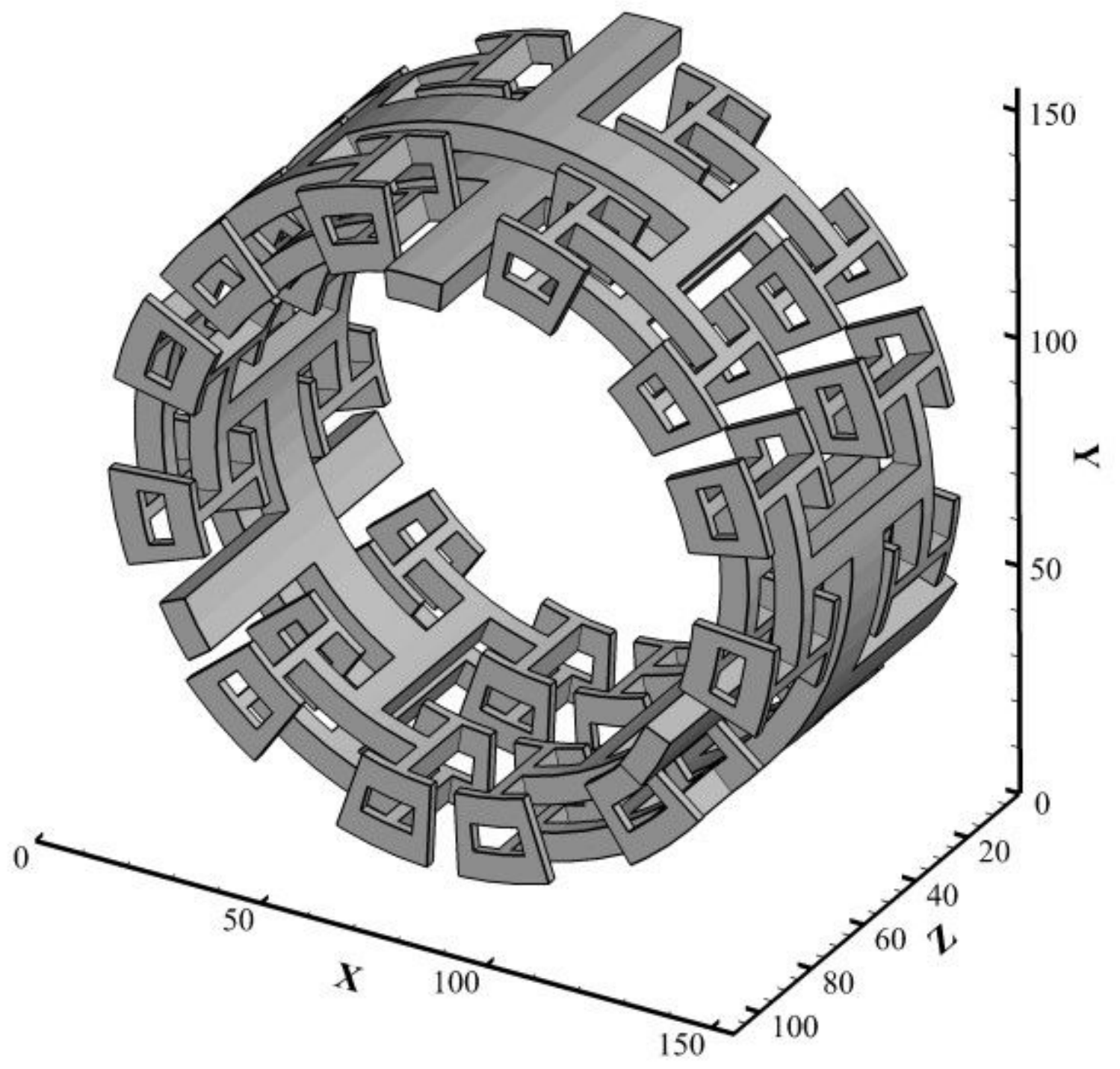

Figure 6

Tree-shaped microchannel network distribution map 


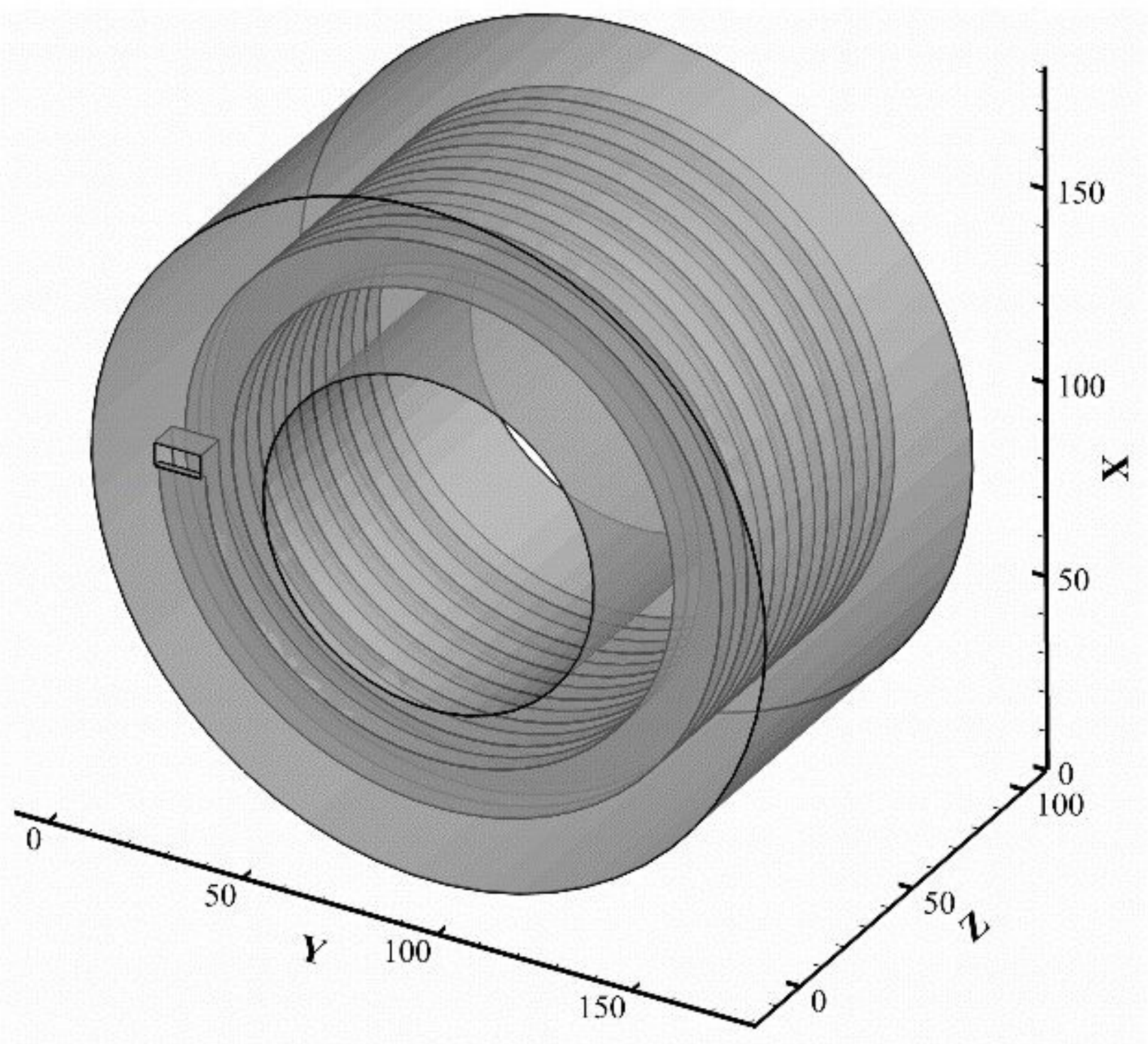

Figure 7

The conventional spiral thermal exchanger 


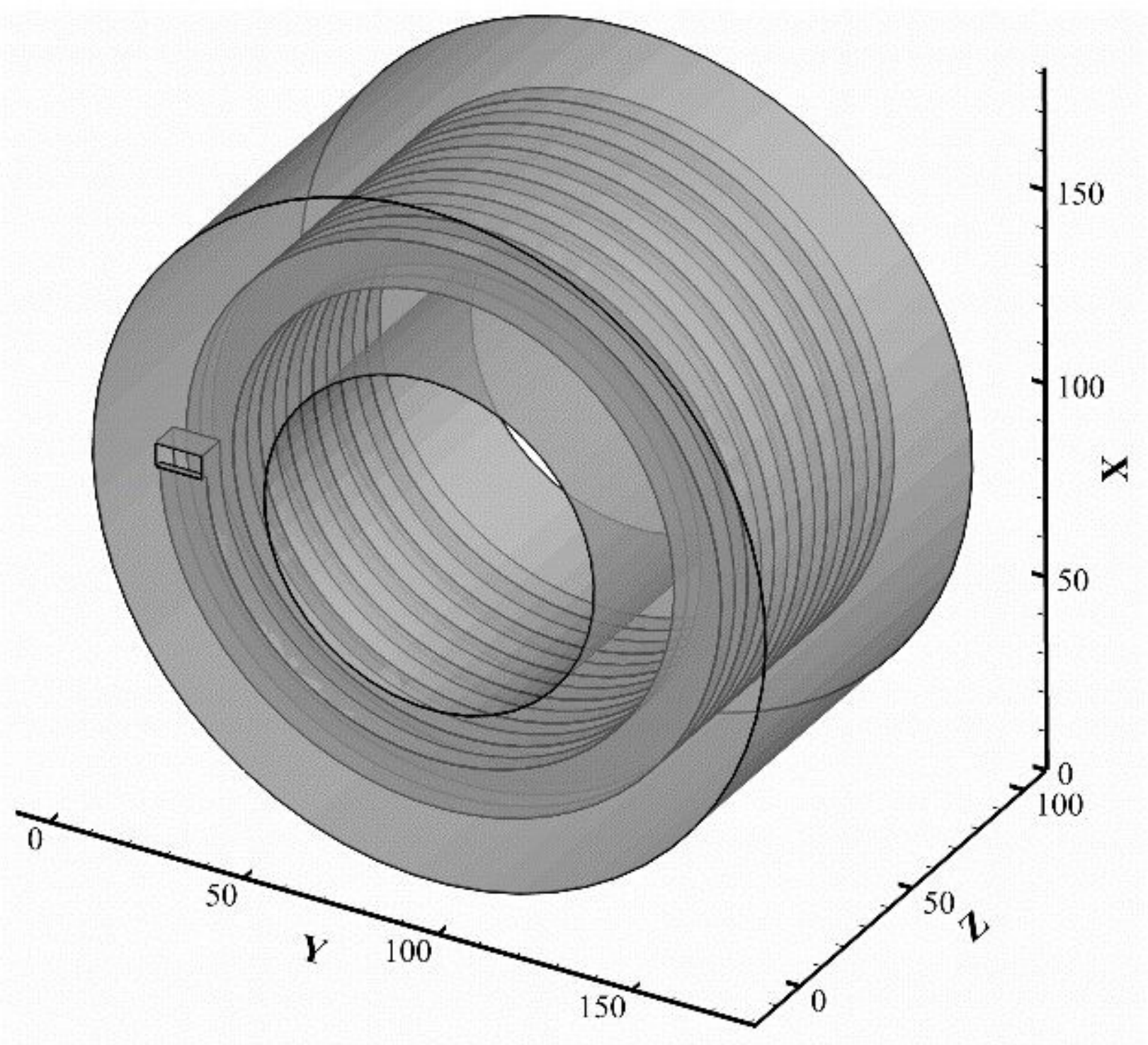

Figure 7

The conventional spiral thermal exchanger 


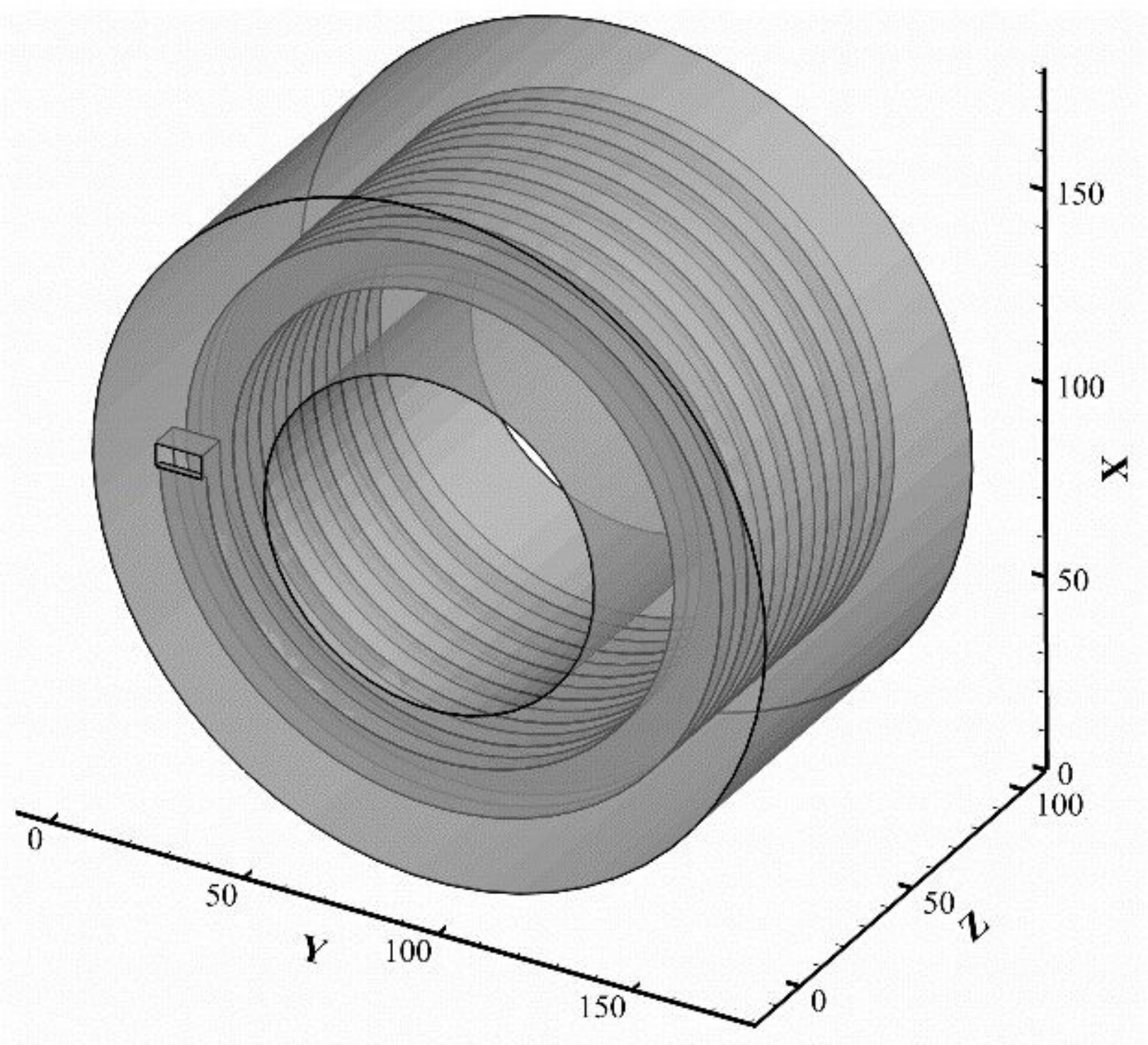

Figure 7

The conventional spiral thermal exchanger 


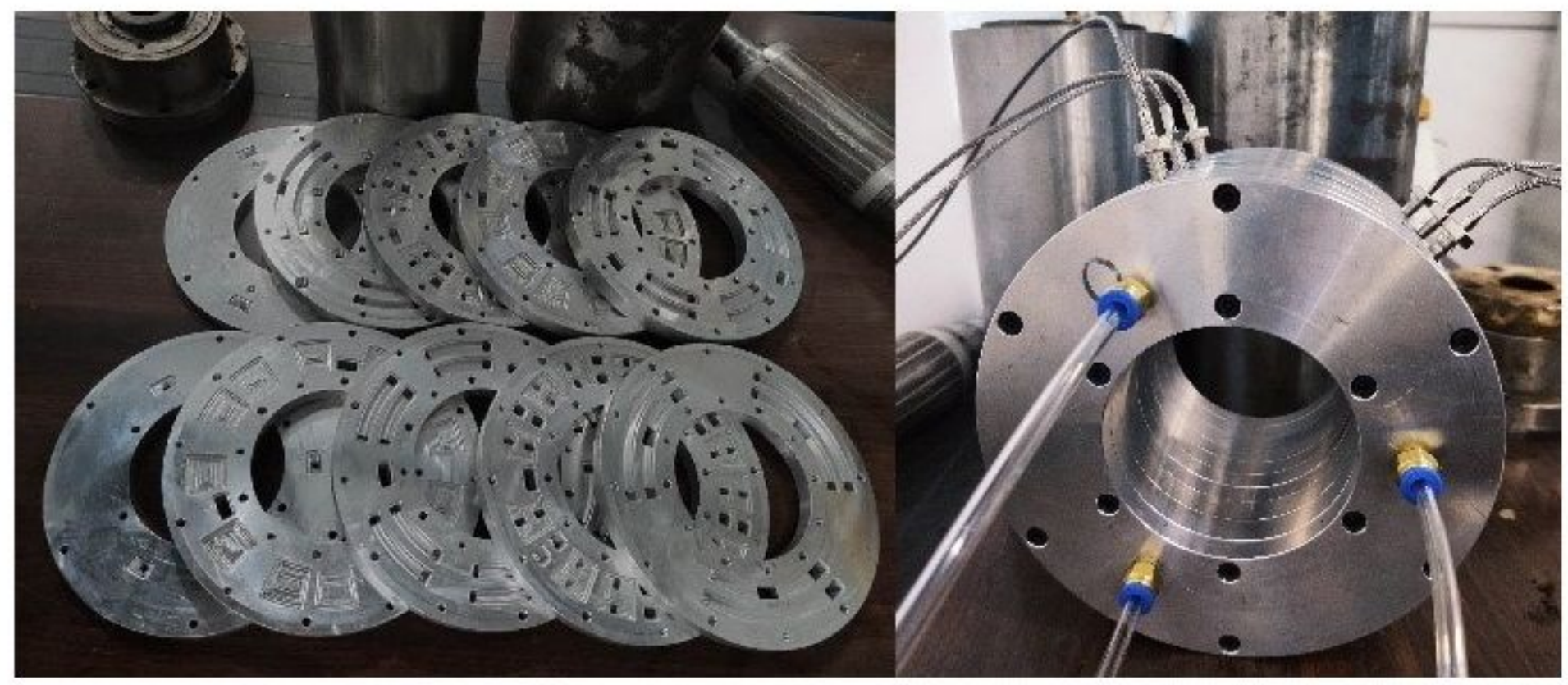

Figure 8

The tree-shaped thermal exchanger structure

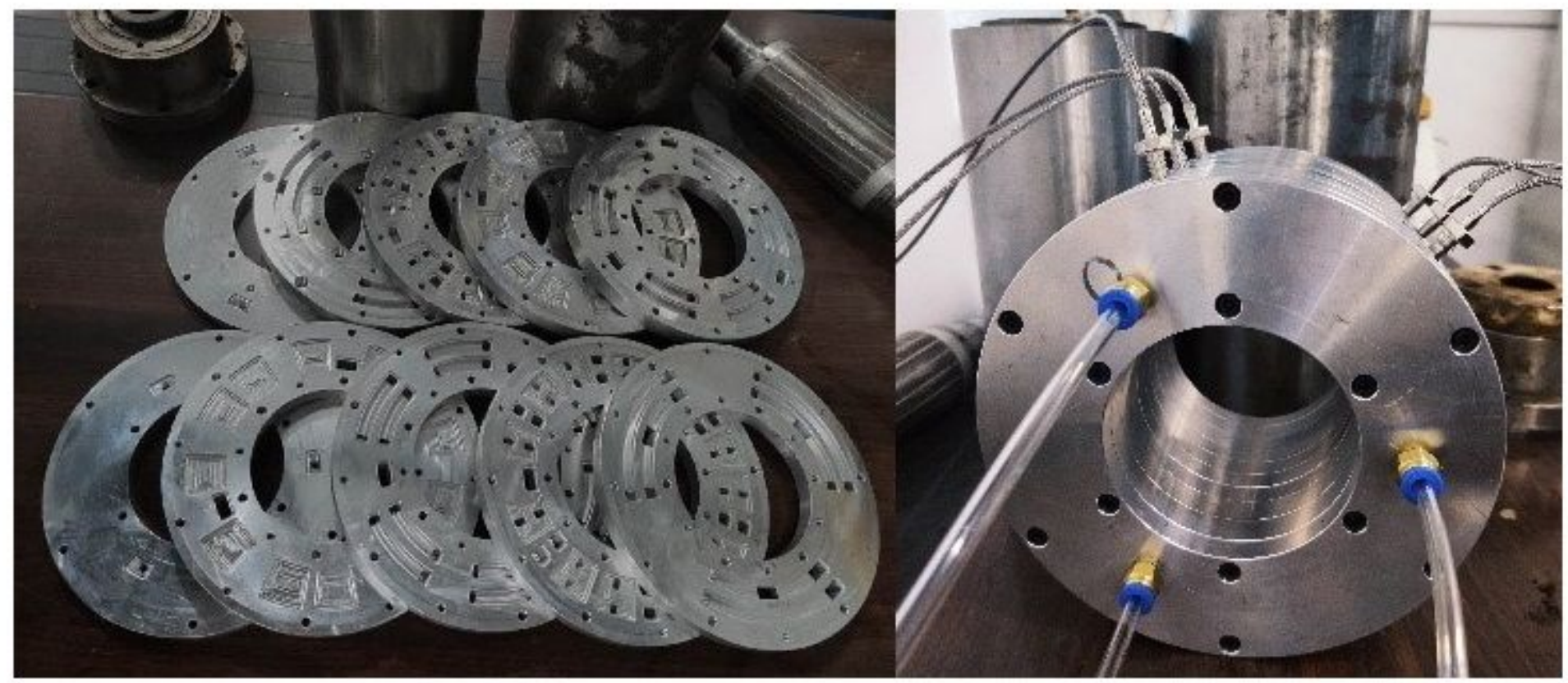

Figure 8

The tree-shaped thermal exchanger structure 


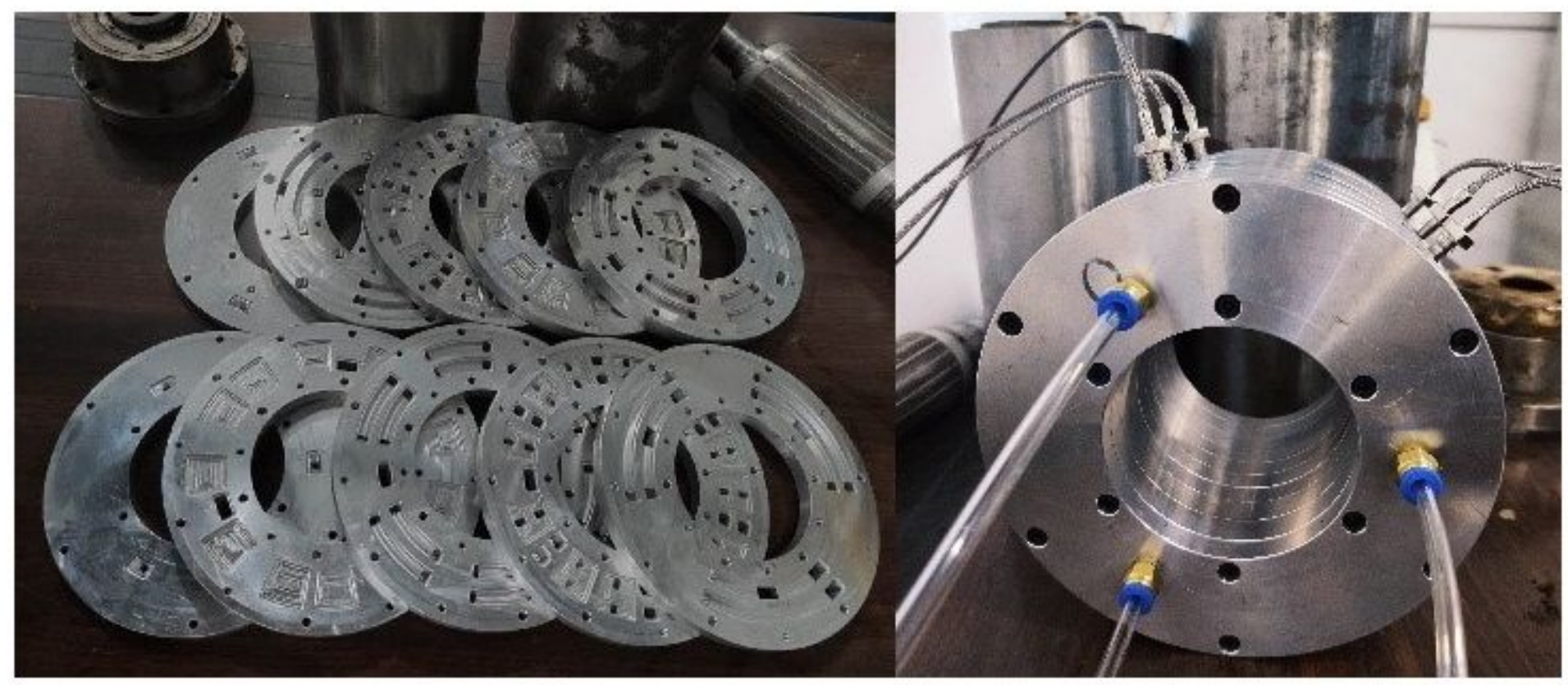

Figure 8

The tree-shaped thermal exchanger structure 

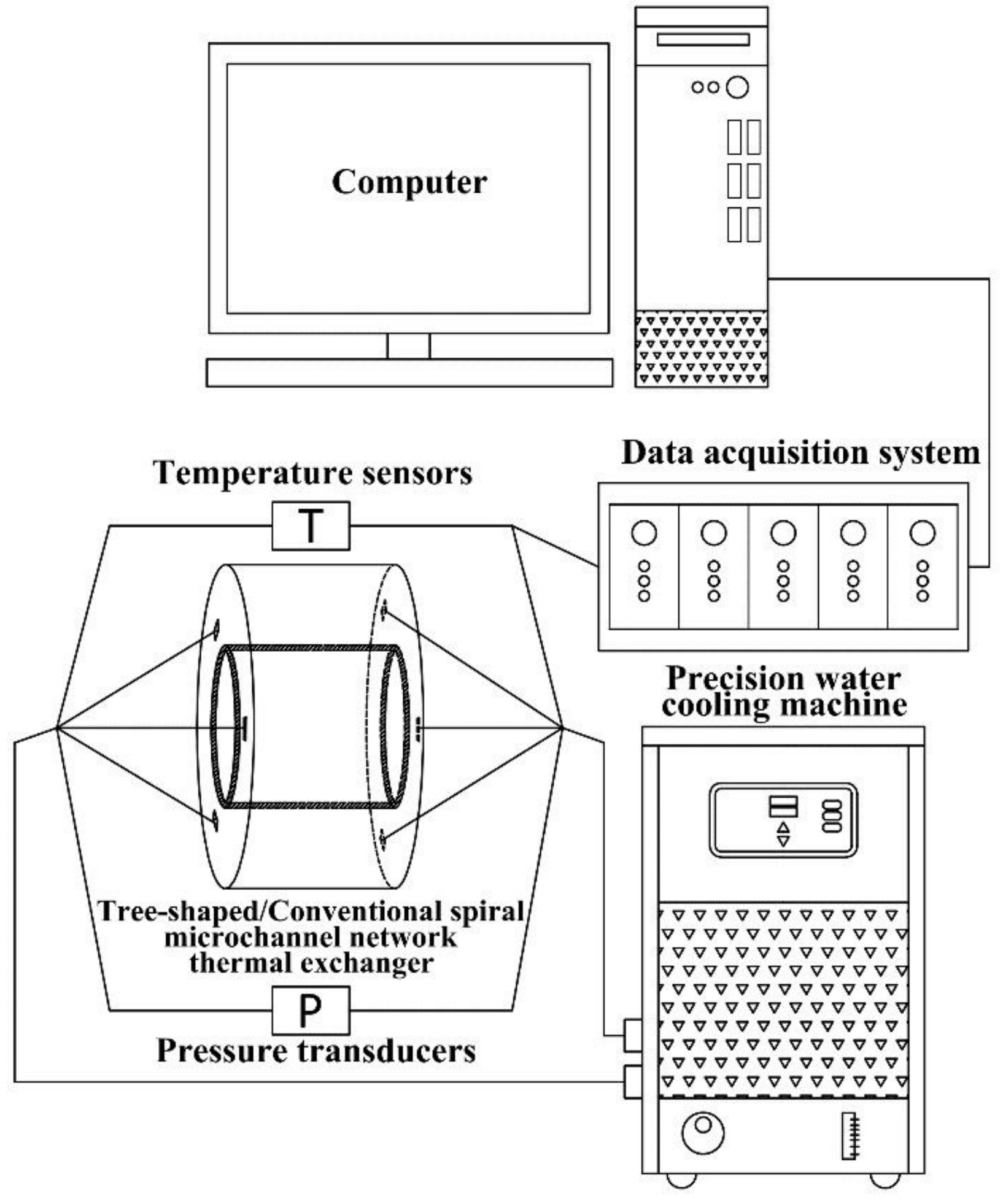

Figure 9

The setup scheme of the experimental device 

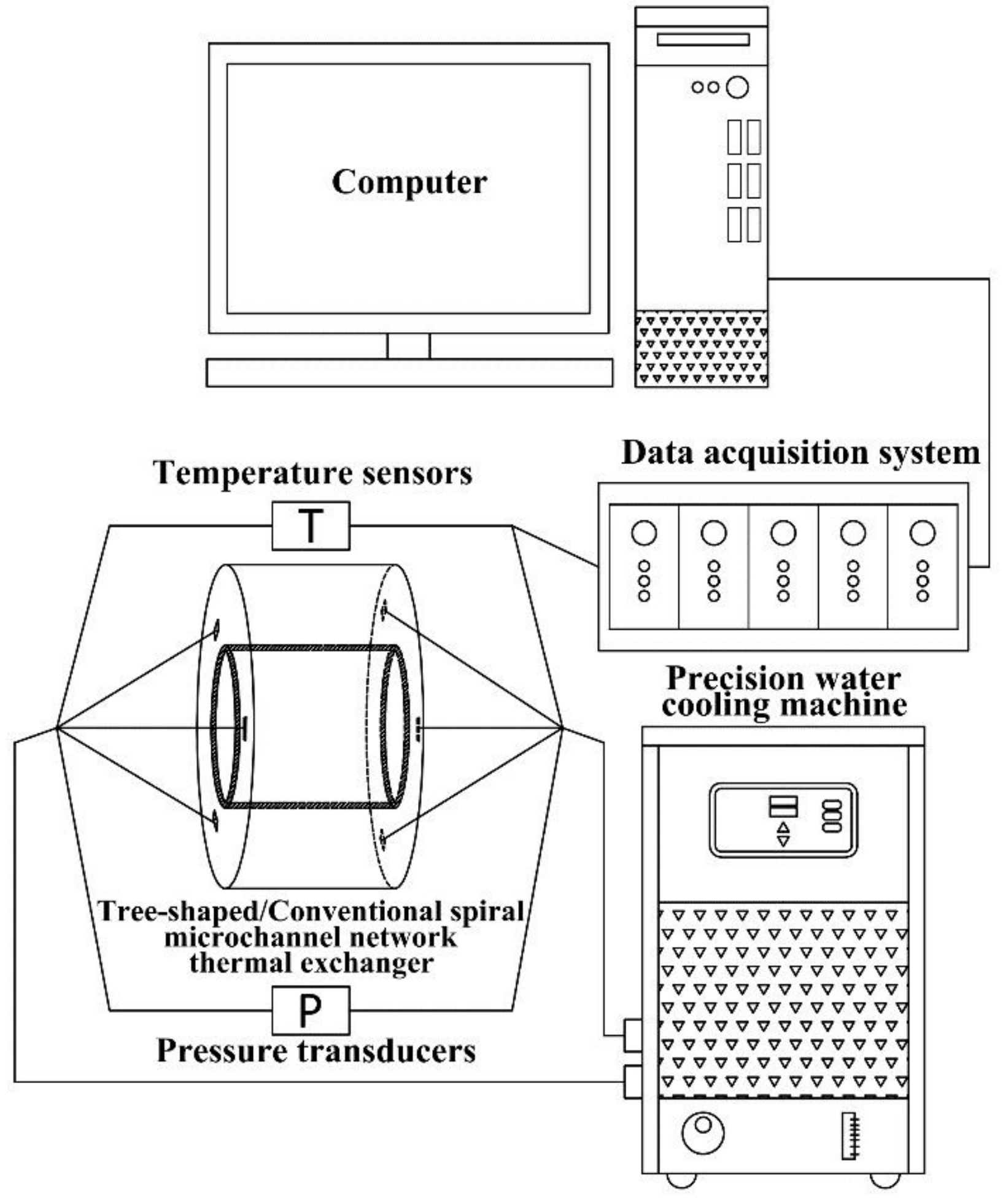

Figure 9

The setup scheme of the experimental device 

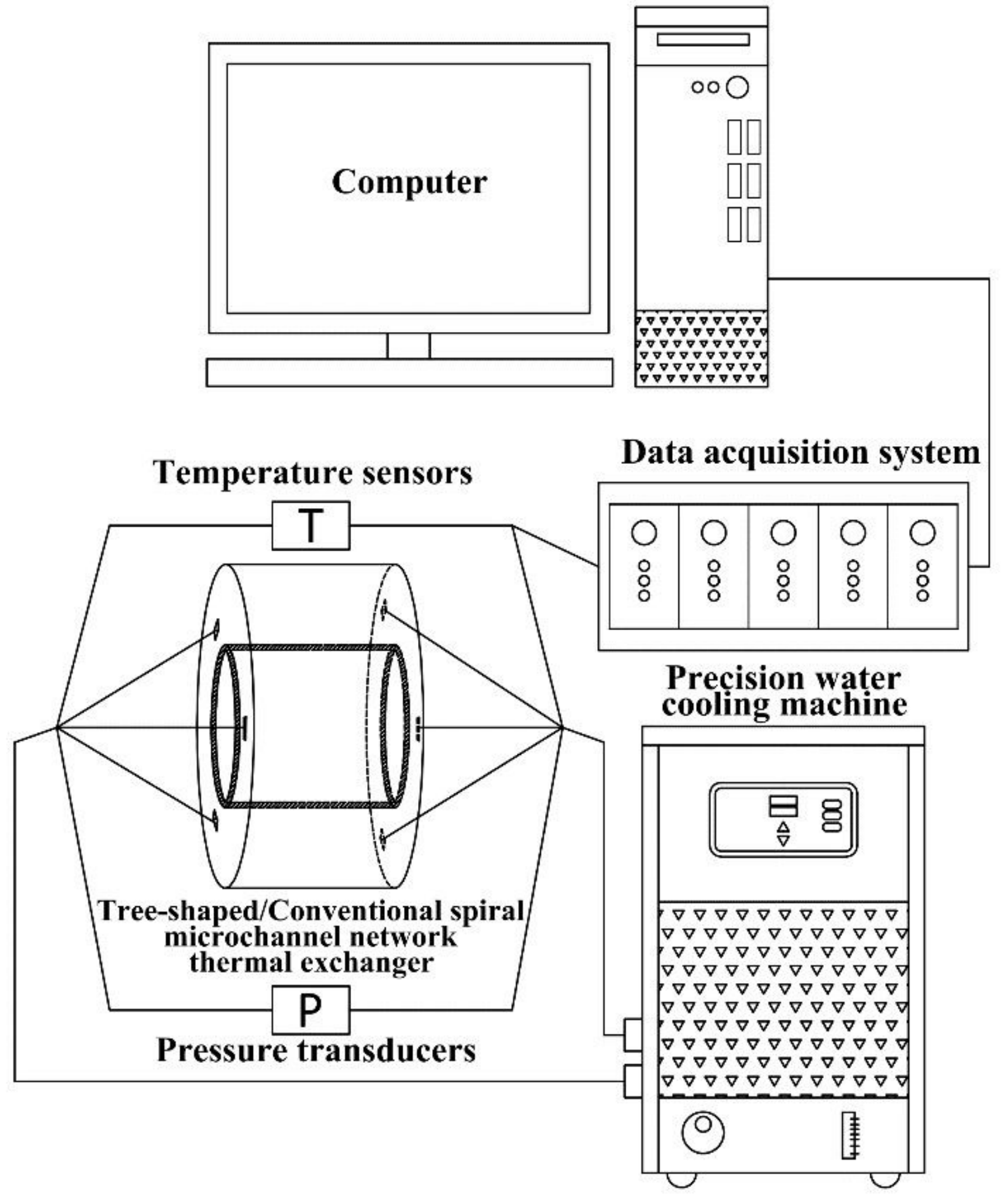

Figure 9

The setup scheme of the experimental device 


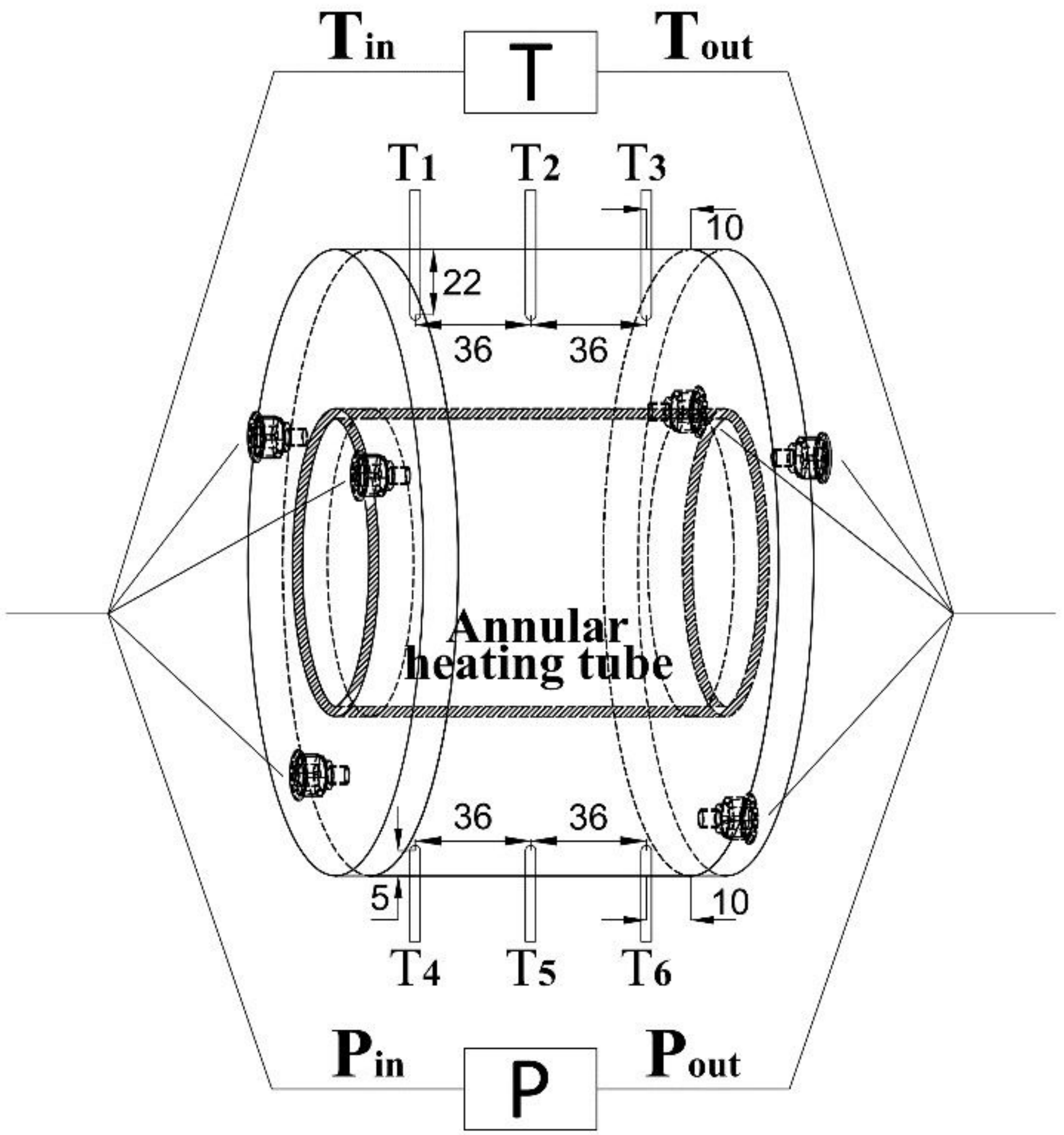

Figure 10

The specific layout of the sensors 


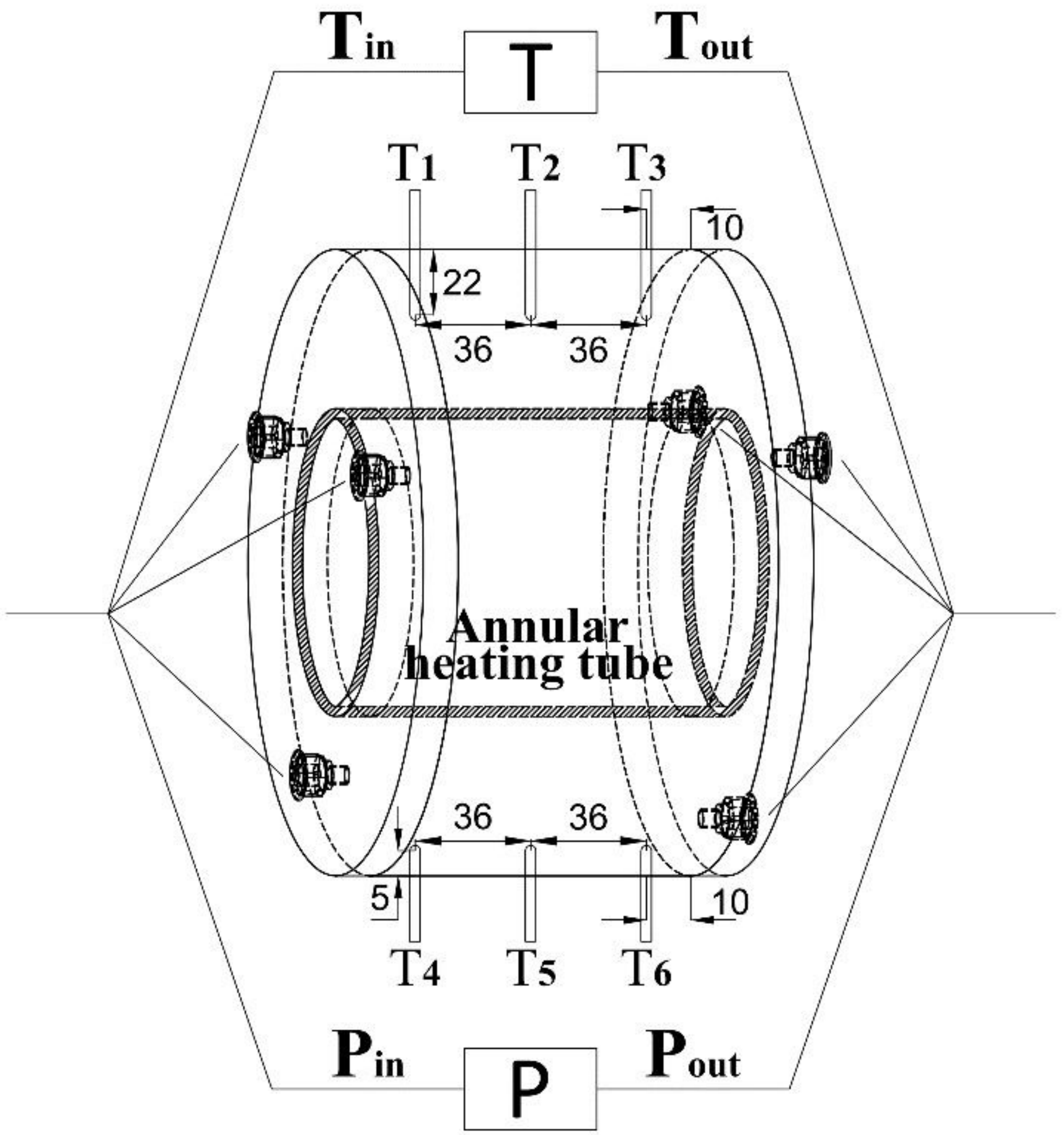

Figure 10

The specific layout of the sensors 


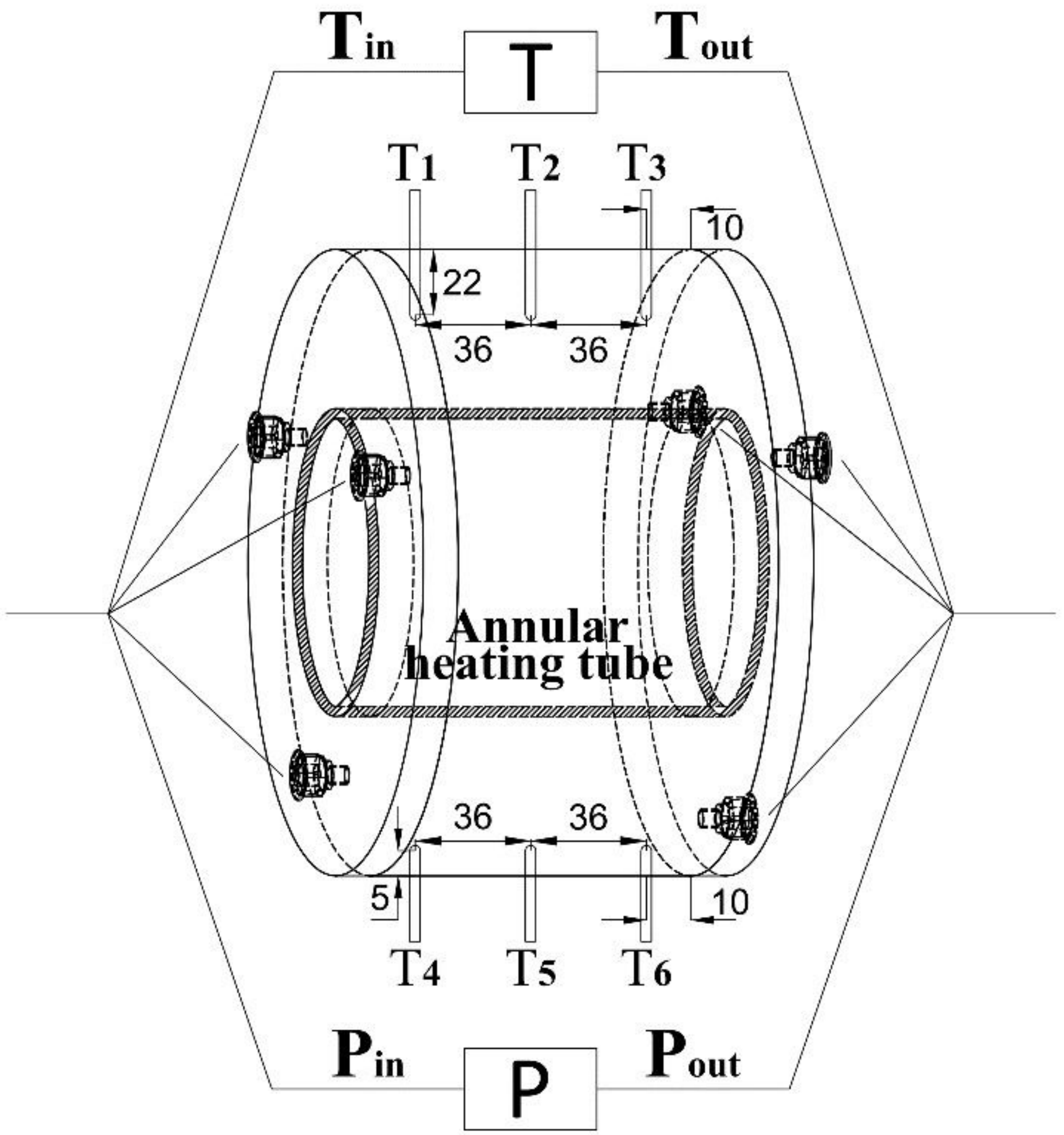

Figure 10

The specific layout of the sensors 

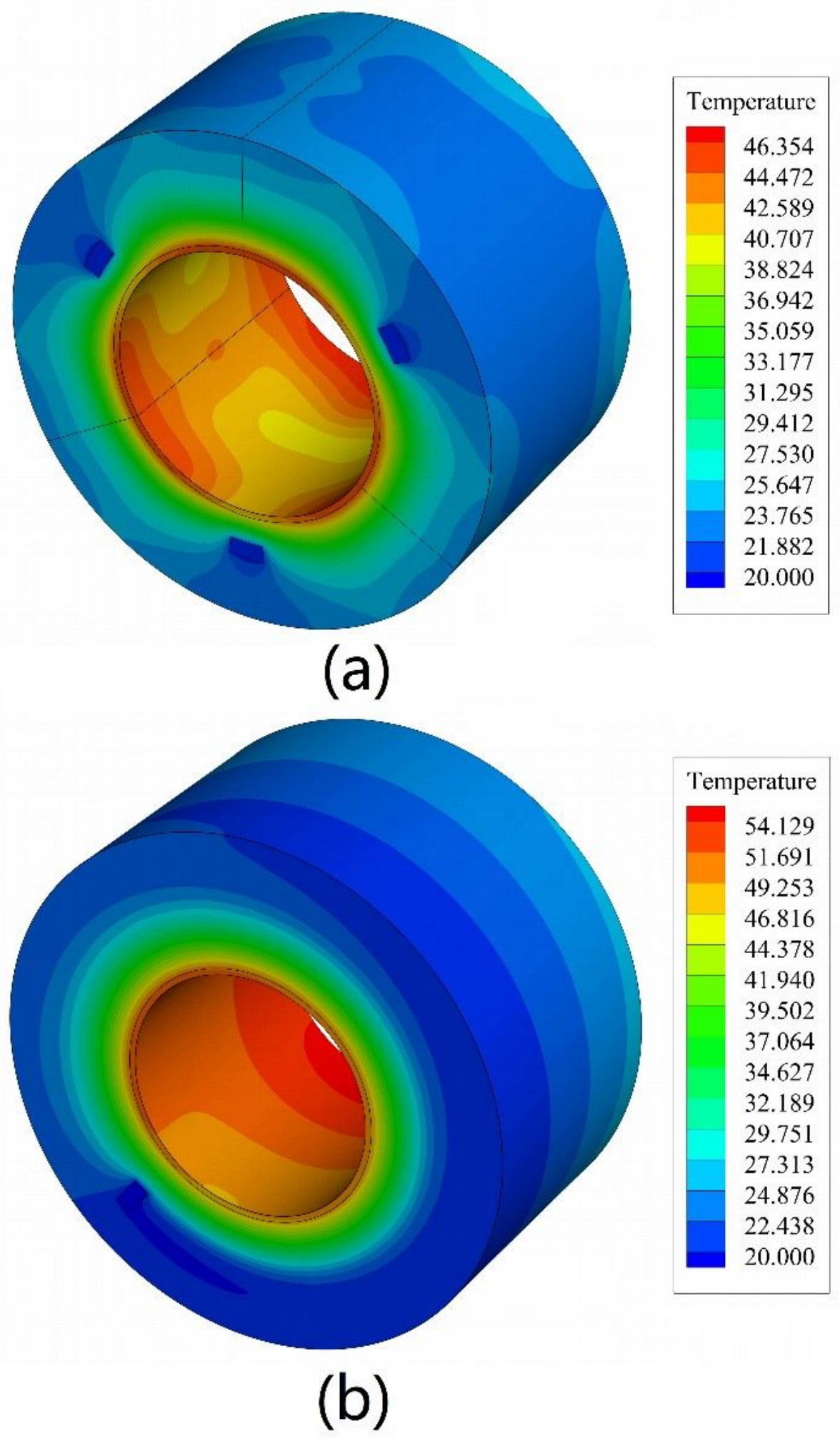

Figure 11

Comparison of thermal field distribution (a) Thermal field distribution of tree-shaped thermal exchanger (b) Thermal field distribution of conventional spiral thermal exchanger 

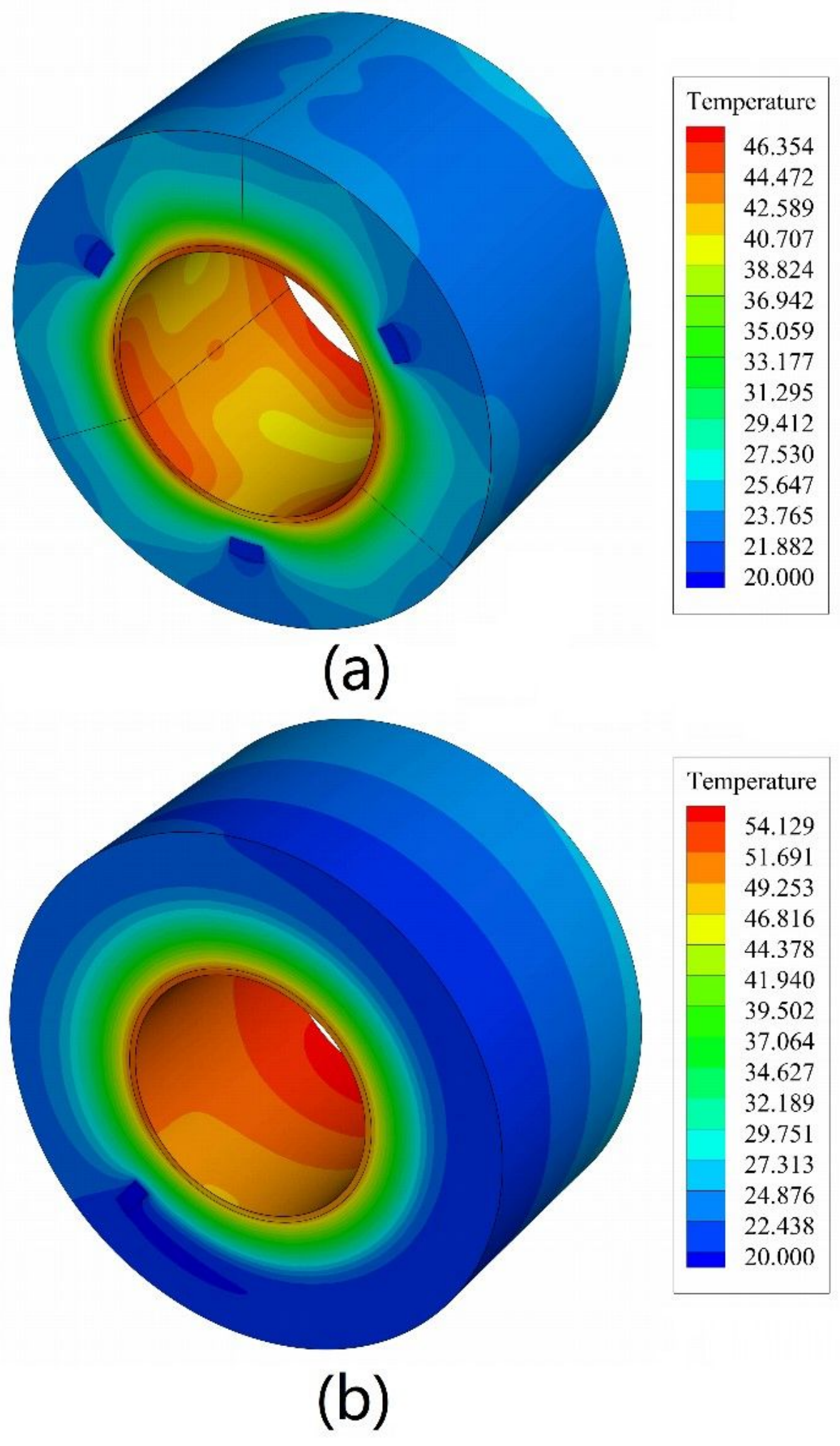

Figure 11

Comparison of thermal field distribution (a) Thermal field distribution of tree-shaped thermal exchanger (b) Thermal field distribution of conventional spiral thermal exchanger 

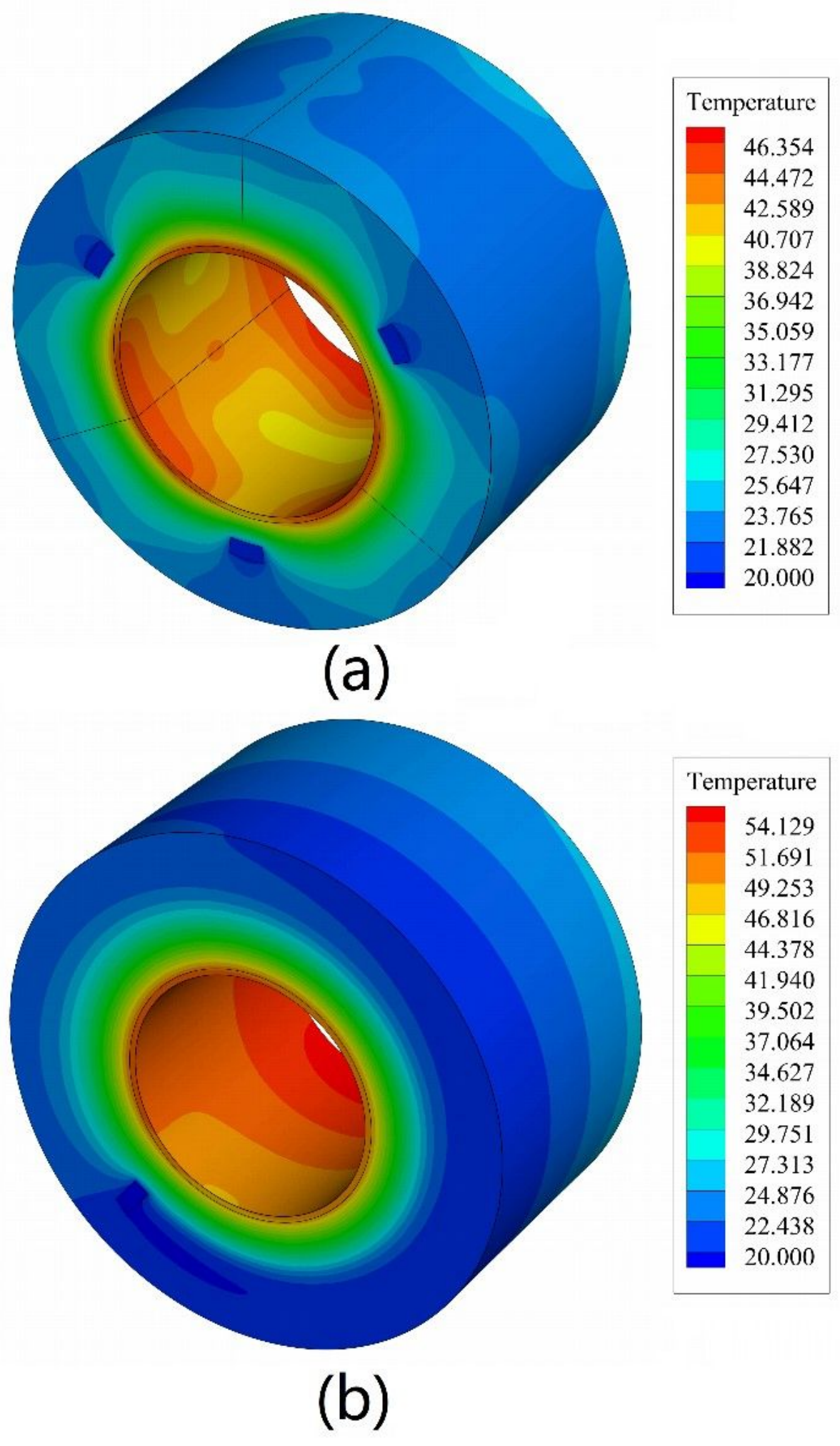

Figure 11

Comparison of thermal field distribution (a) Thermal field distribution of tree-shaped thermal exchanger (b) Thermal field distribution of conventional spiral thermal exchanger 


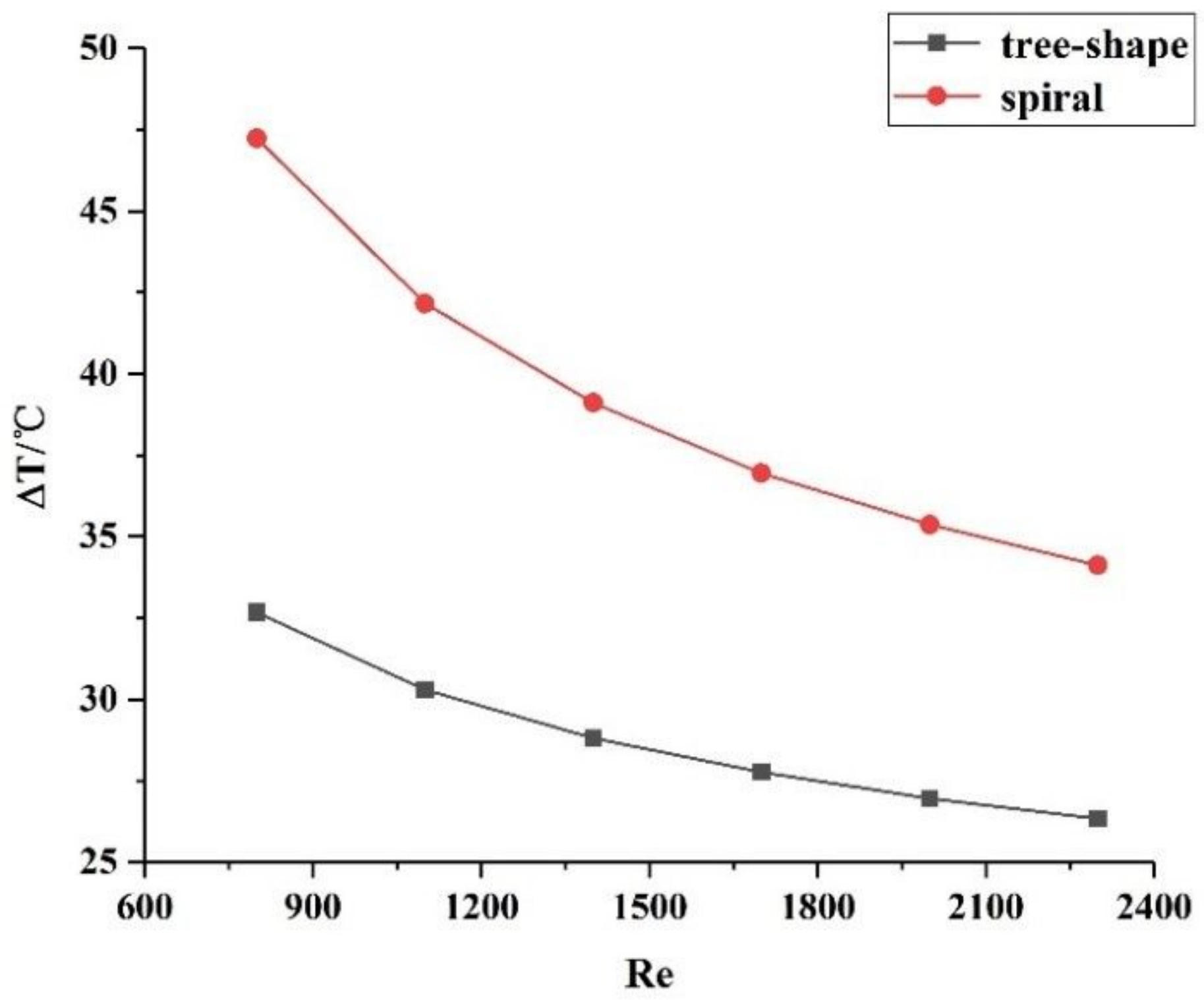

Figure 12

Comparison of temperature gradient between tree-shaped thermal exchanger and spiral thermal exchanger 


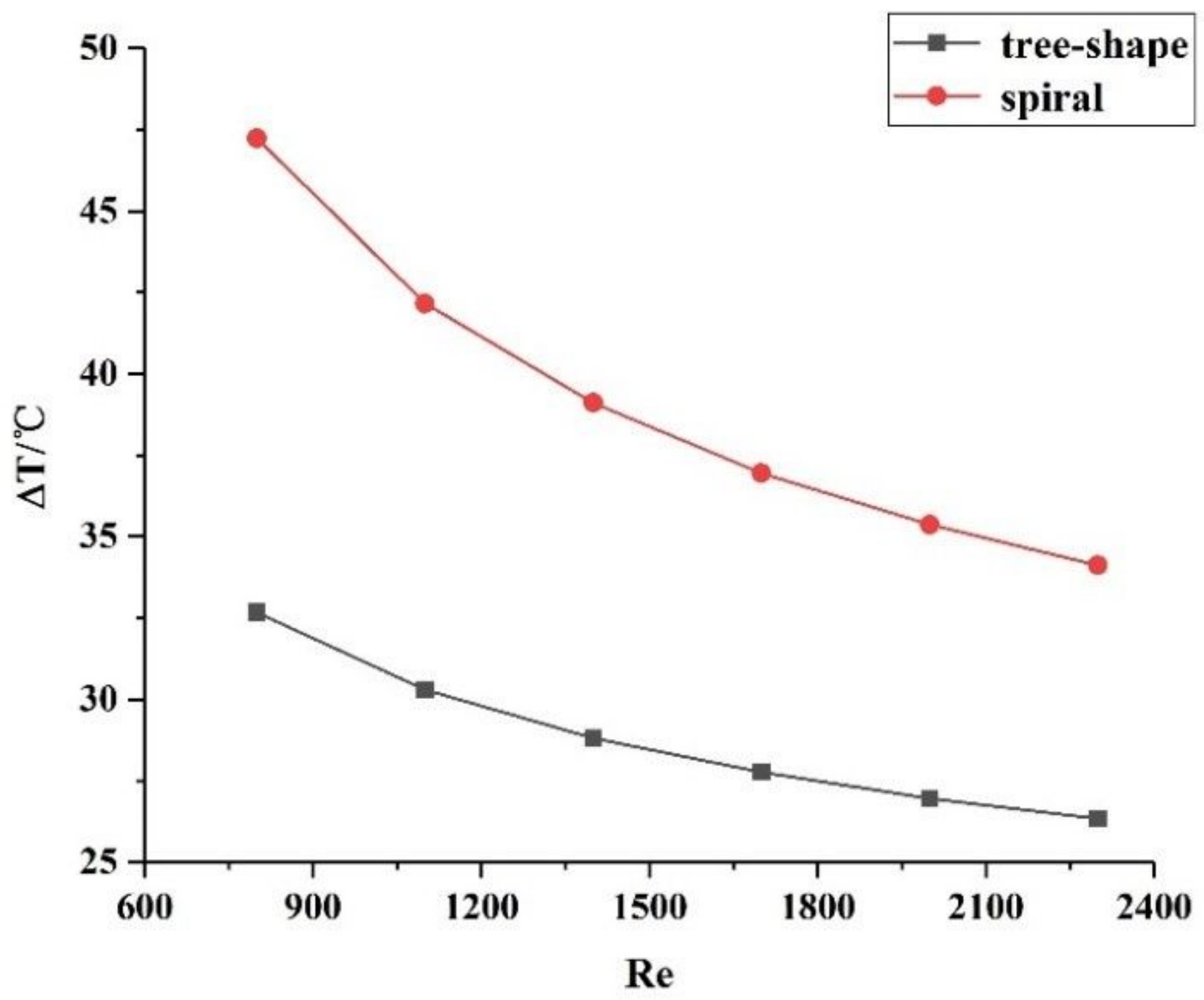

Figure 12

Comparison of temperature gradient between tree-shaped thermal exchanger and spiral thermal exchanger 


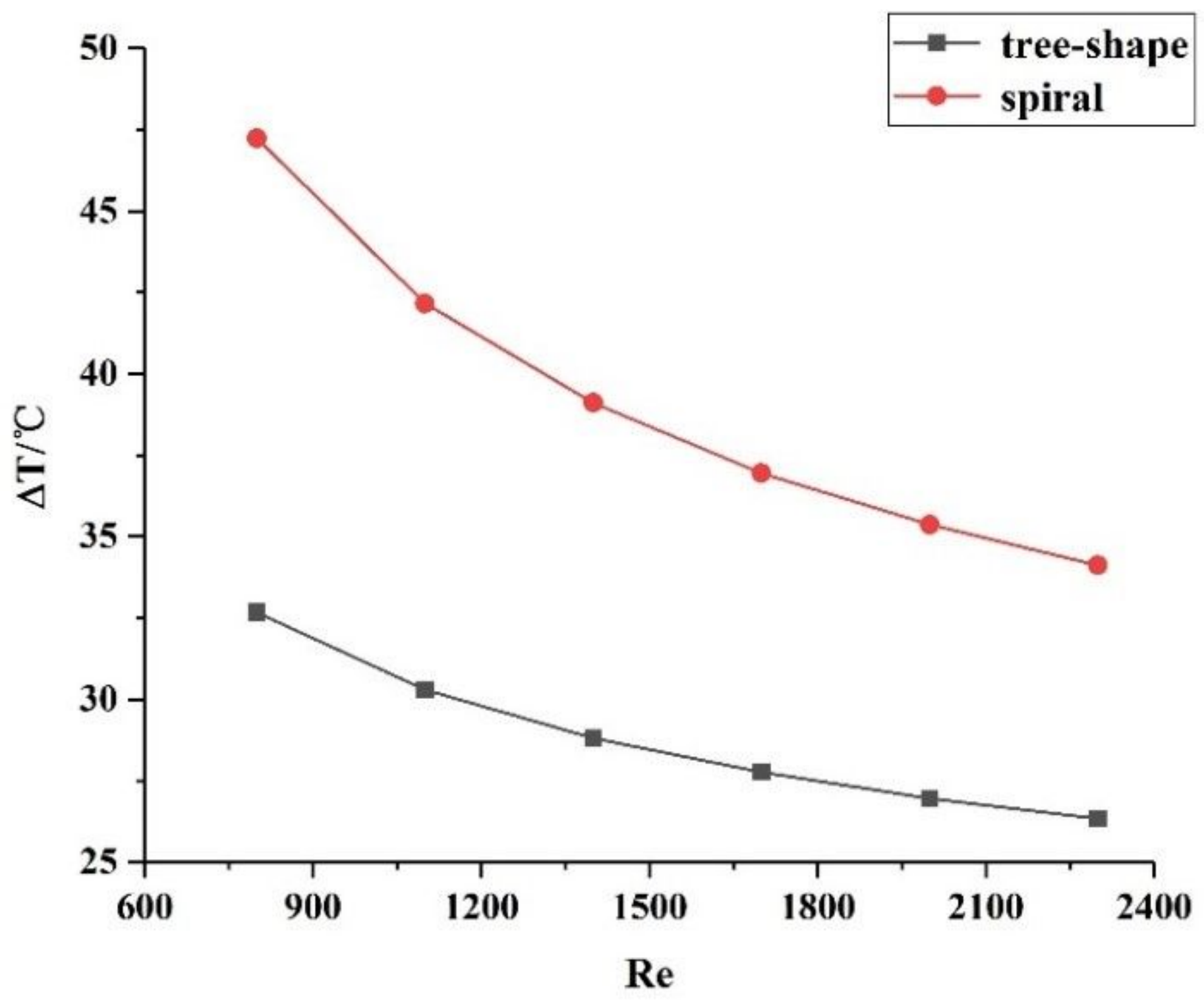

Figure 12

Comparison of temperature gradient between tree-shaped thermal exchanger and spiral thermal exchanger 


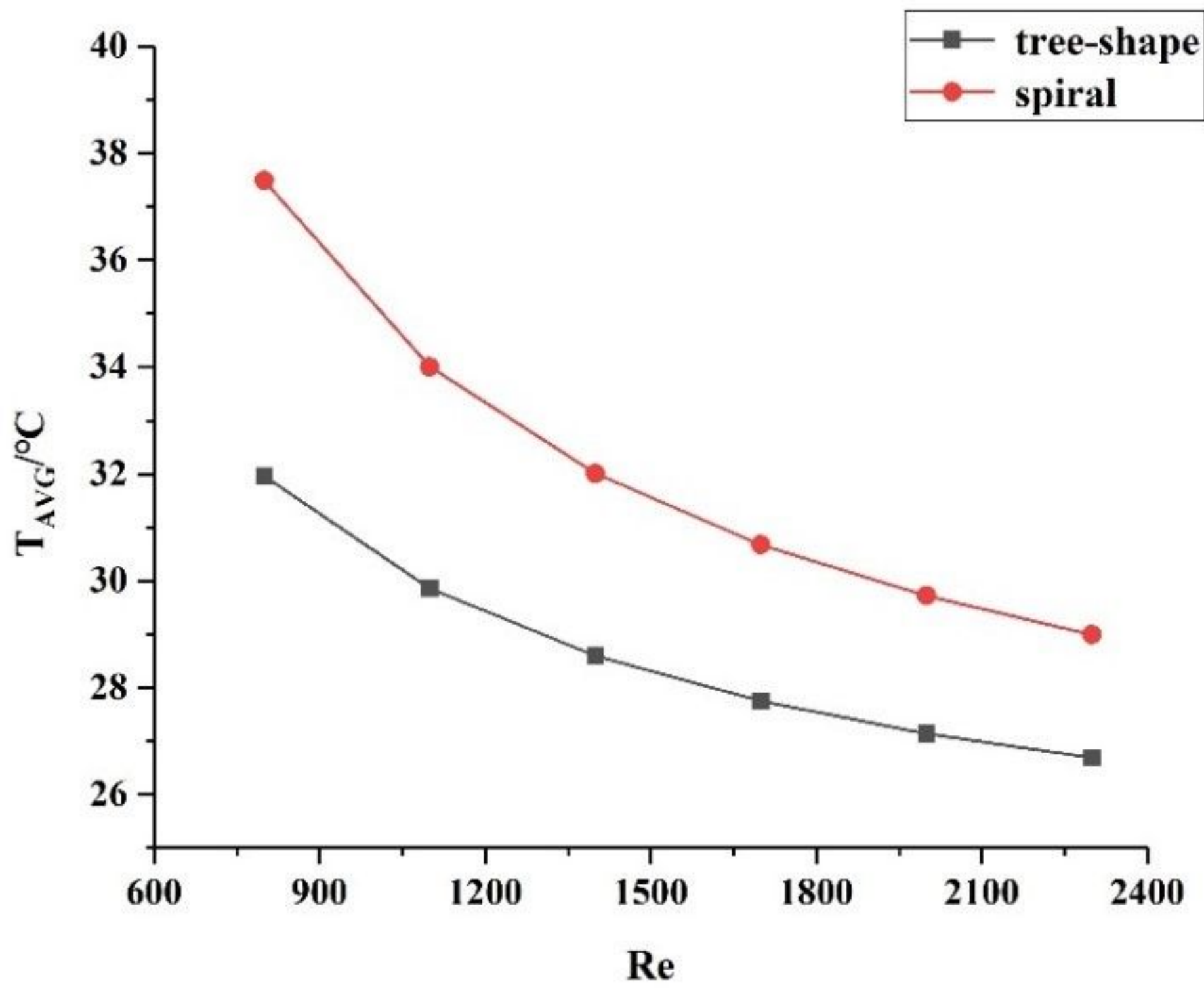

Figure 13

Comparison of average temperature between tree-shaped thermal exchanger and spiral thermal exchanger 


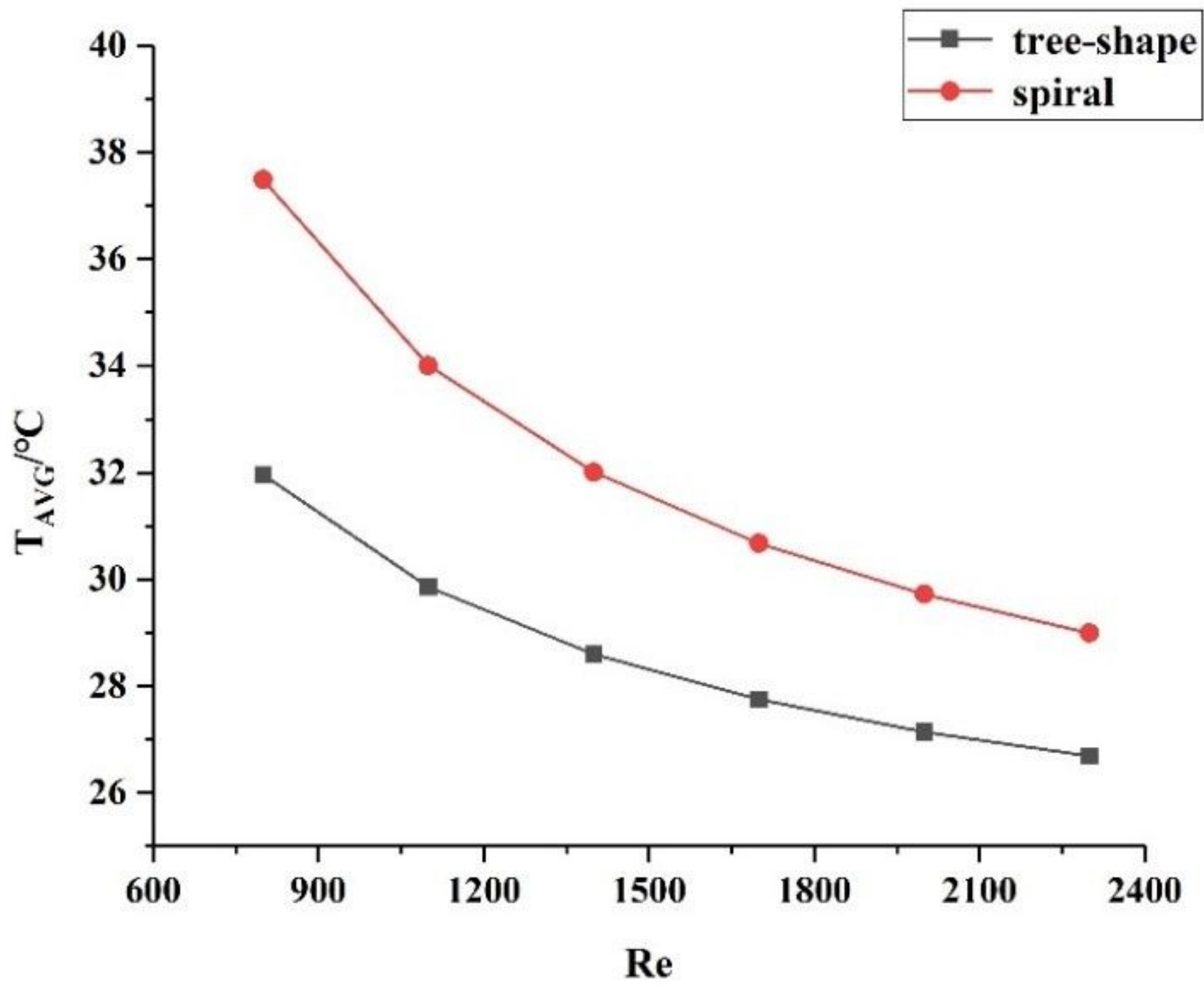

Figure 13

Comparison of average temperature between tree-shaped thermal exchanger and spiral thermal exchanger 


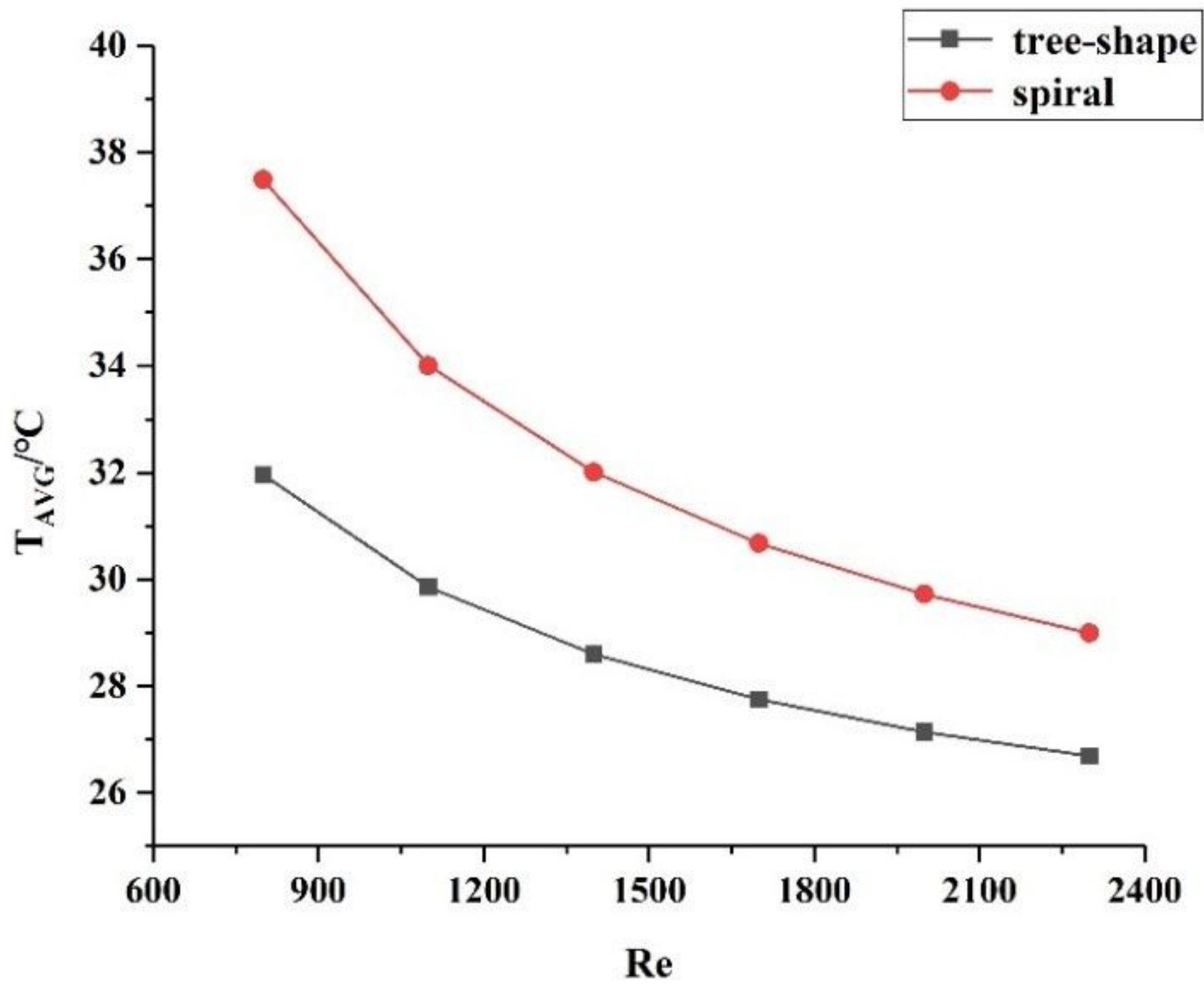

Figure 13

Comparison of average temperature between tree-shaped thermal exchanger and spiral thermal exchanger 


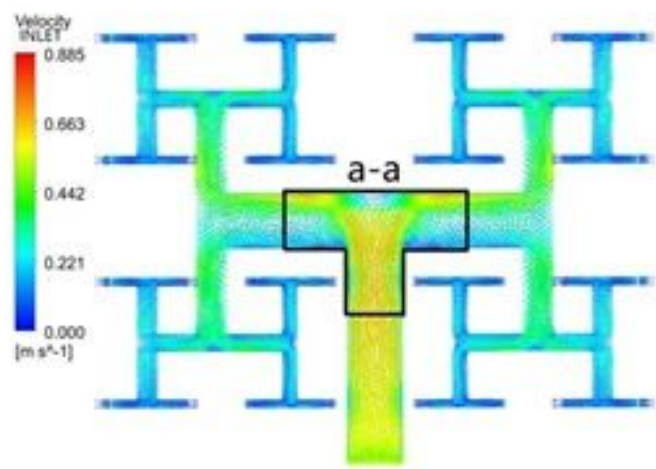

(a)

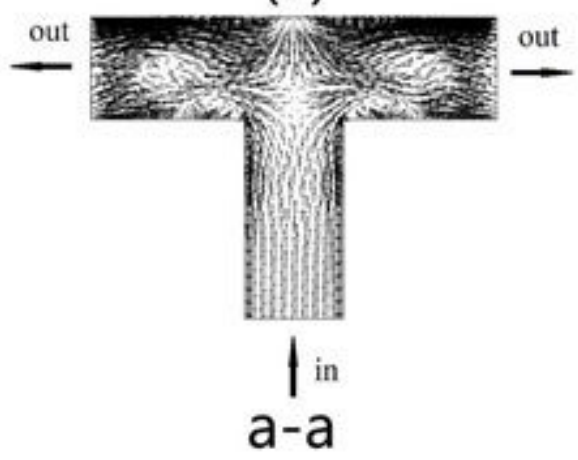

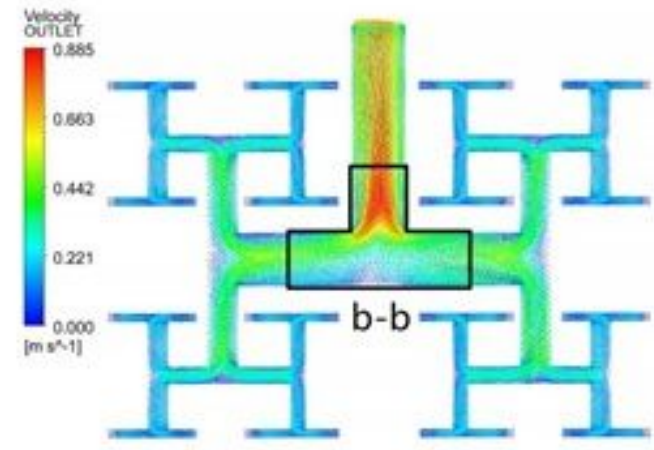

(b)

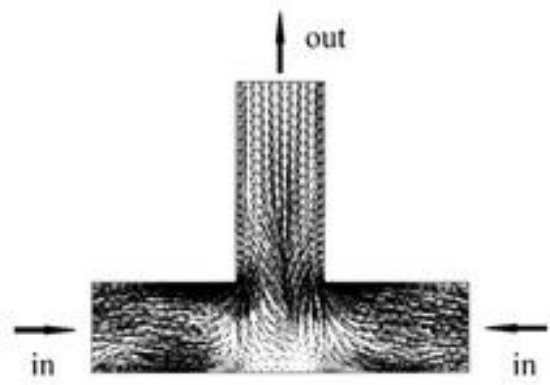

$b-b$

Figure 14

Laminar mixing in tree-shaped thermal exchanger (a) Flow condition of tree-shaped thermal exchanger at $\mathrm{R}=59 \mathrm{~mm}$ (b) Flow condition of tree-shaped thermal exchanger at $\mathrm{R}=71 \mathrm{~mm}$ a-a. The vortex at the inner corner of the afflux channel b-b. The vortex of the efflux channel

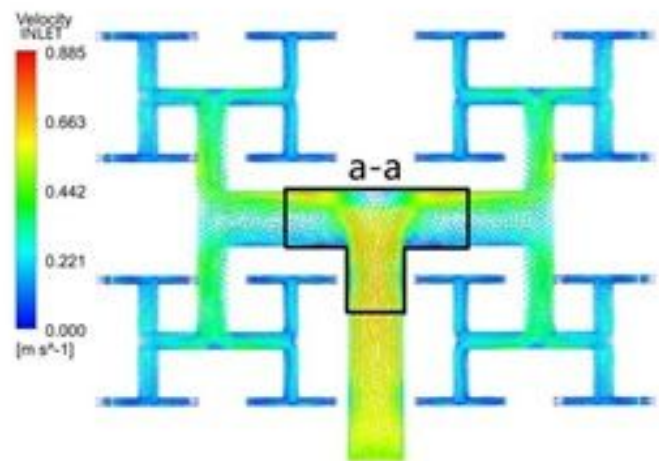

(a)

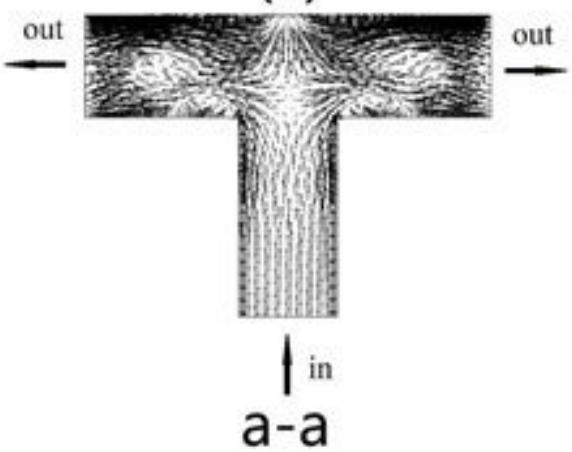

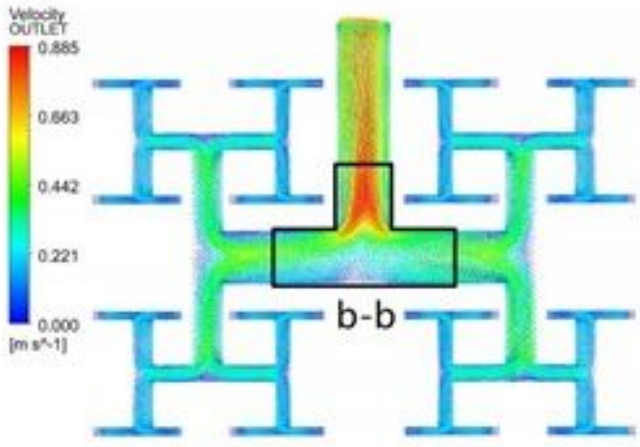

(b)

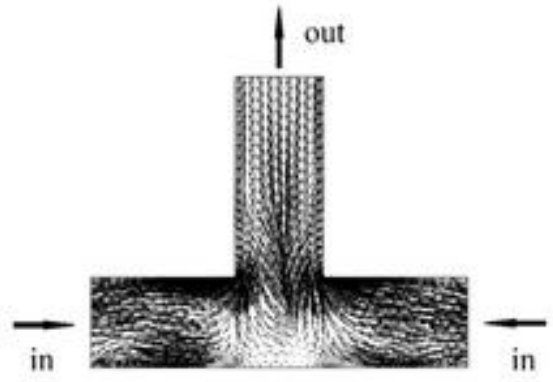

$b-b$ 
Figure 14

Laminar mixing in tree-shaped thermal exchanger (a) Flow condition of tree-shaped thermal exchanger at $\mathrm{R}=59 \mathrm{~mm}$ (b) Flow condition of tree-shaped thermal exchanger at $\mathrm{R}=71 \mathrm{~mm}$ a-a. The vortex at the inner corner of the afflux channel $b-b$. The vortex of the efflux channel

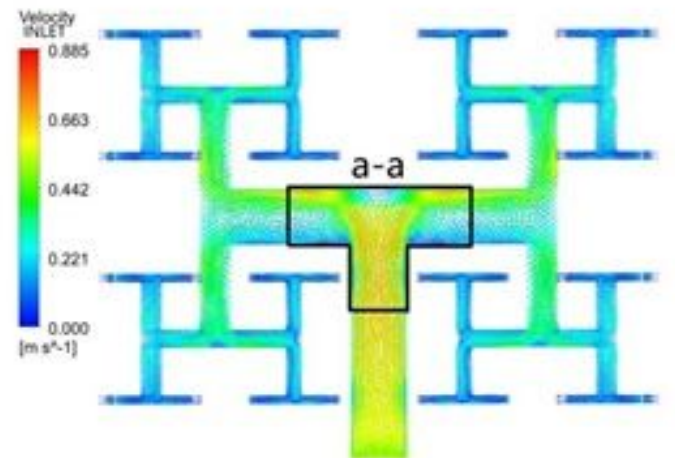

(a)

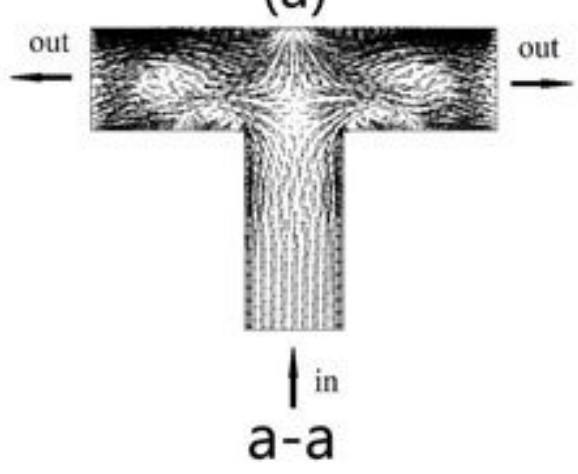

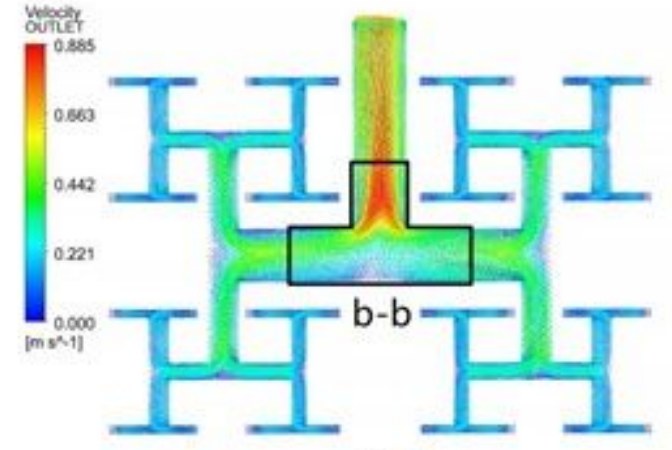

(b)

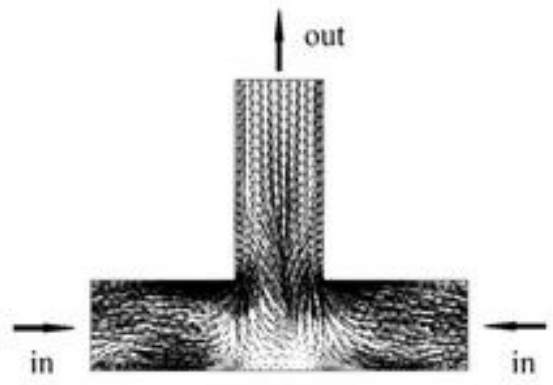

$b-b$

Figure 14

Laminar mixing in tree-shaped thermal exchanger (a) Flow condition of tree-shaped thermal exchanger at $\mathrm{R}=59 \mathrm{~mm}$ (b) Flow condition of tree-shaped thermal exchanger at $\mathrm{R}=71 \mathrm{~mm}$ a-a. The vortex at the inner corner of the afflux channel b-b. The vortex of the efflux channel 


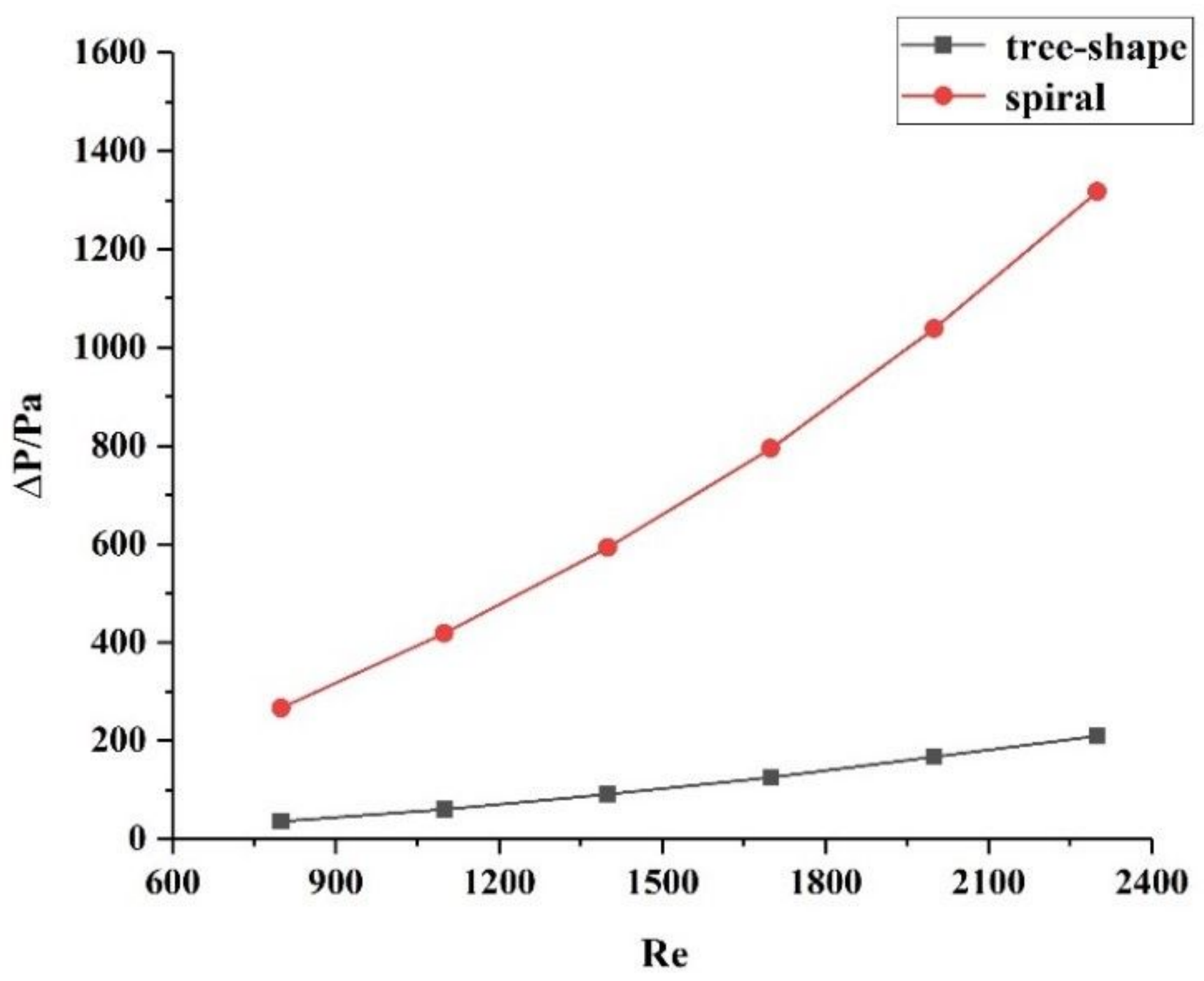

Figure 15

Comparison of pressure drop between tree-shaped thermal exchanger and spiral thermal exchanger 


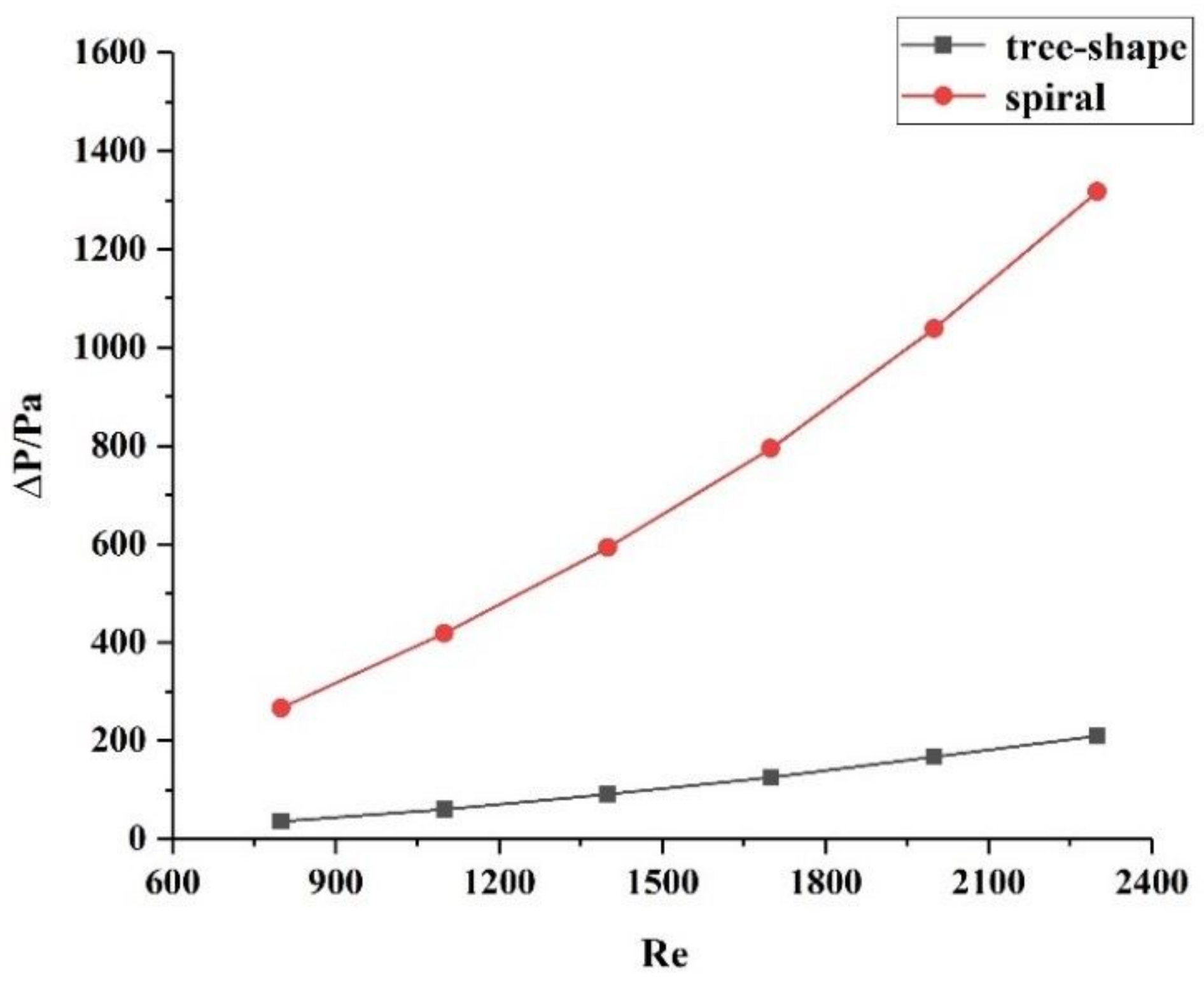

Figure 15

Comparison of pressure drop between tree-shaped thermal exchanger and spiral thermal exchanger 


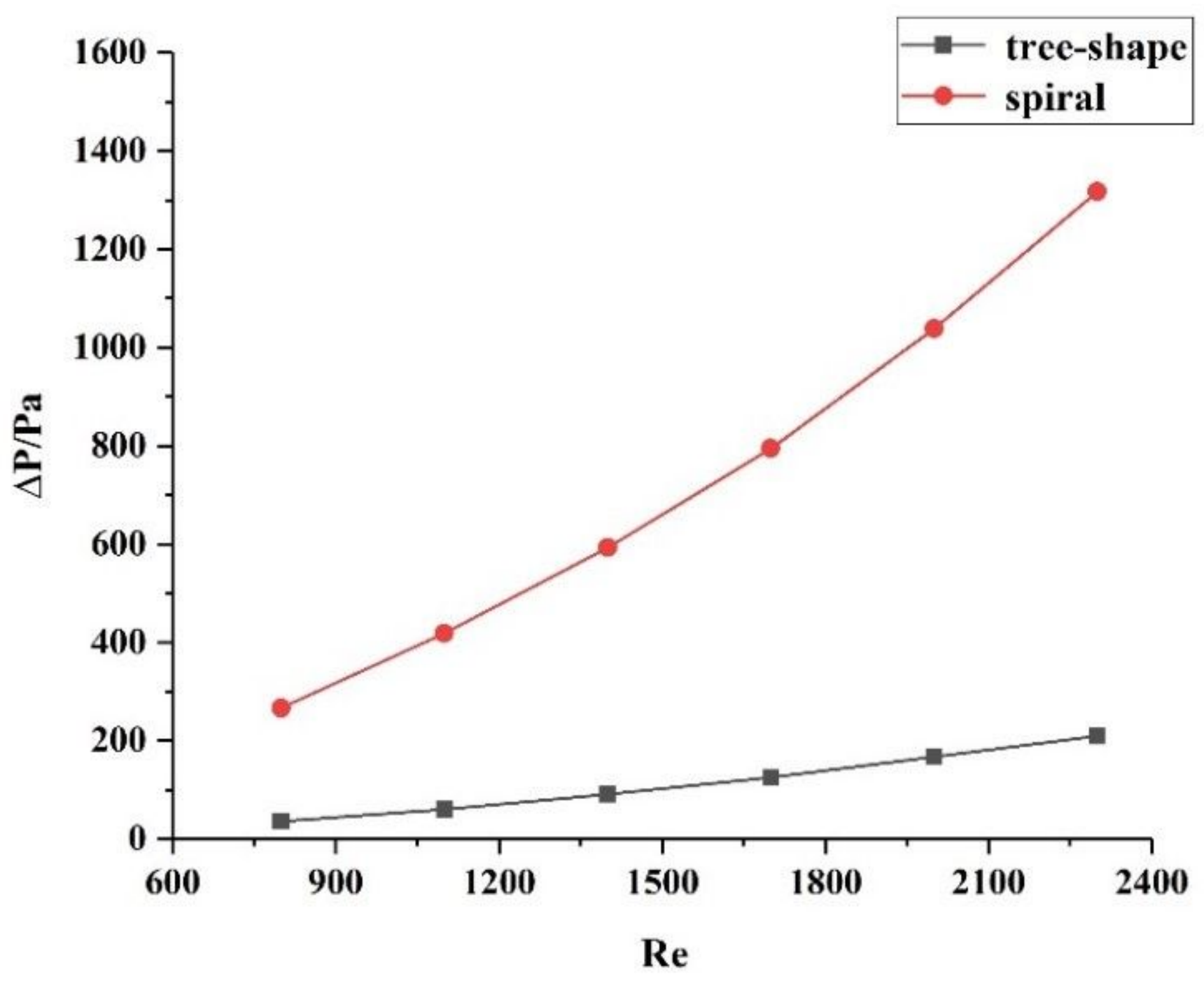

Figure 15

Comparison of pressure drop between tree-shaped thermal exchanger and spiral thermal exchanger 


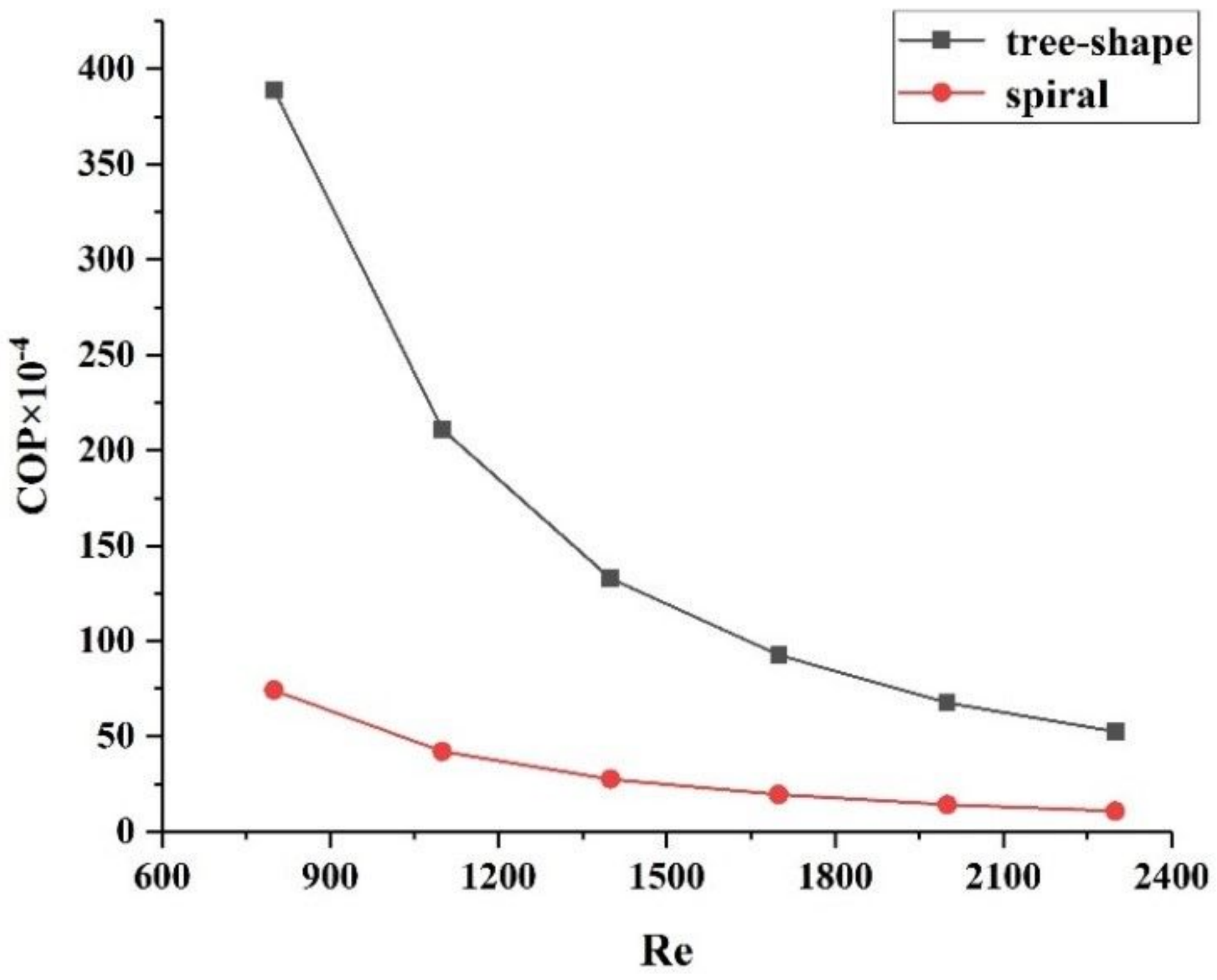

Figure 16

Comparison of performance coefficient between tree-shaped thermal exchanger and spiral thermal exchanger 


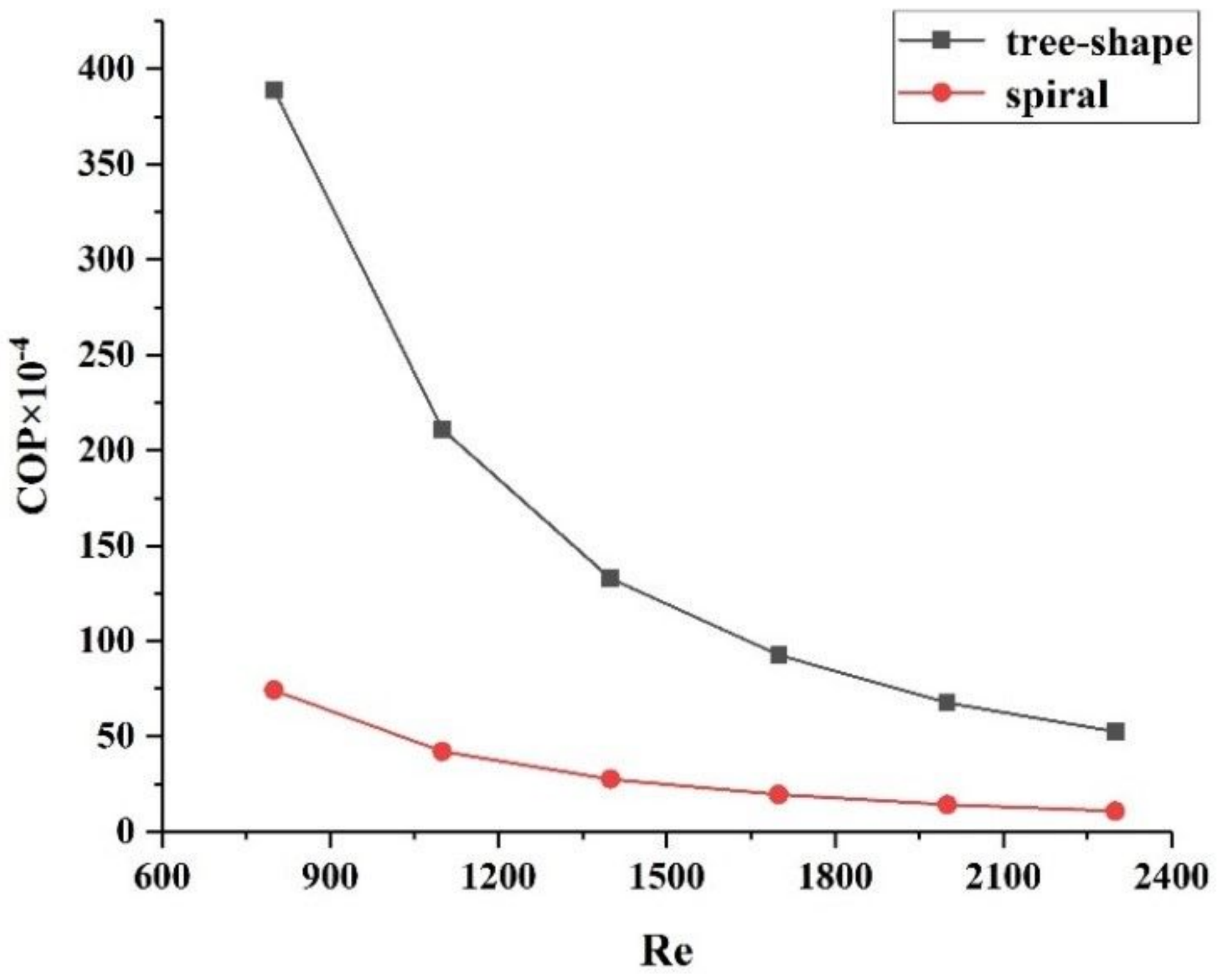

Figure 16

Comparison of performance coefficient between tree-shaped thermal exchanger and spiral thermal exchanger 


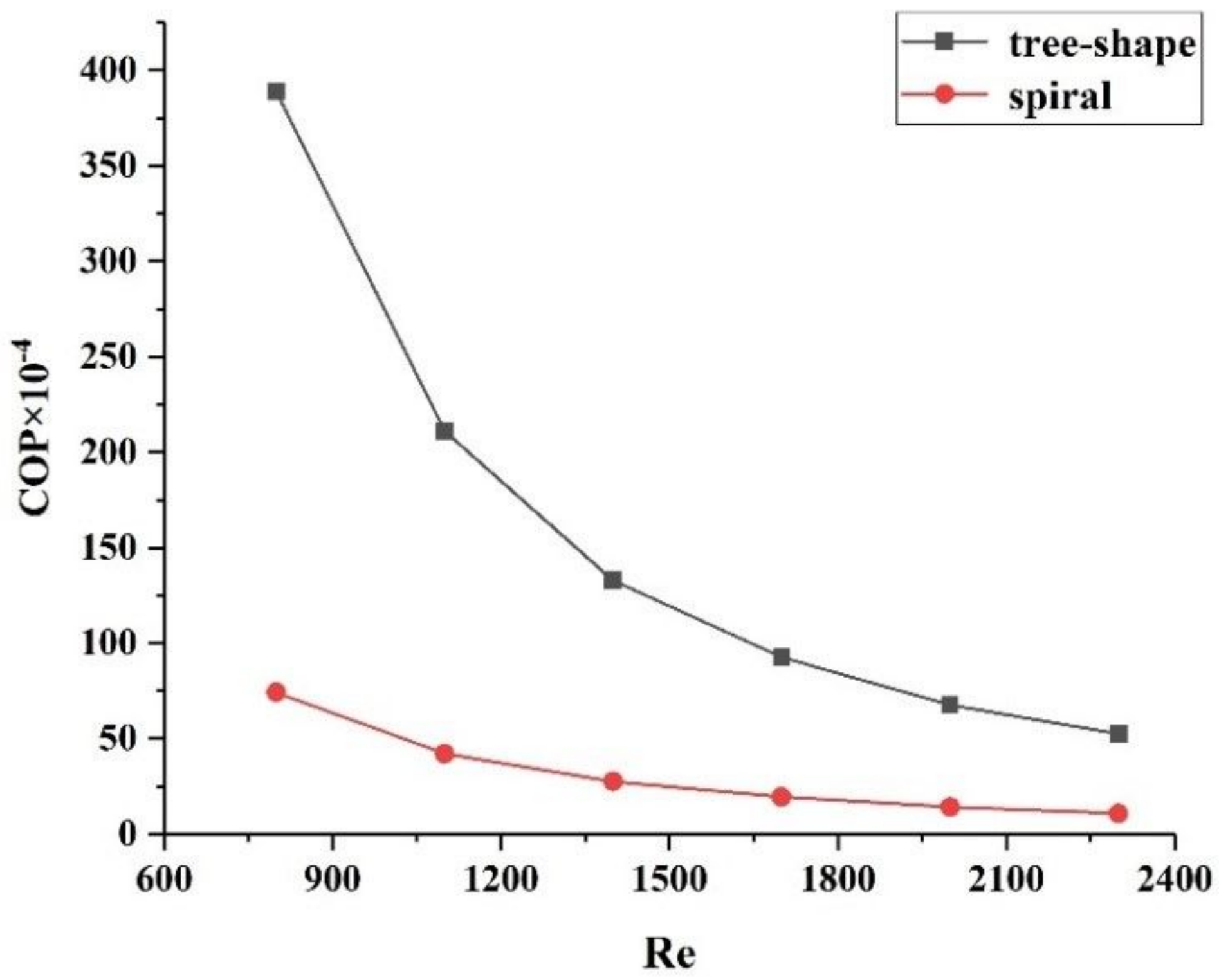

Figure 16

Comparison of performance coefficient between tree-shaped thermal exchanger and spiral thermal exchanger 